\title{
THE DIRECT GROWTH OF CARBON NANOTUBES AND ITS SUBSTRATE
}

\section{DEPENDENCE}

by

Chris Munro

\author{
A thesis \\ submitted to the Victoria University of Wellington \\ in fulfilment of the degree of \\ Master of Science \\ in Chemistry
}

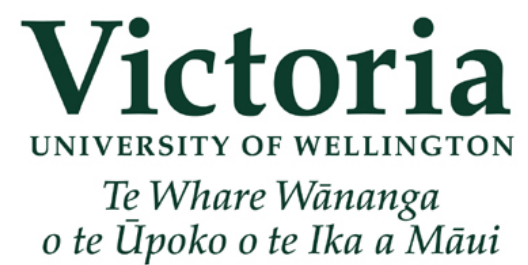

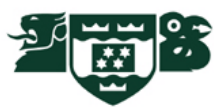




\section{Abbreviations}

$\begin{array}{ll}\text { CNT(s) } & \text { carbon nanotube(s) } \\ \text { CVD } & \text { chemical vapour deposition } \\ \text { MWNT } & \text { multi-walled carbon nanotube } \\ \text { RBM } & \text { radial breathing mode } \\ \text { SWNT } & \text { single-walled carbon nanotube } \\ \text { SEM } & \text { scanning electron microscope } \\ \text { SEI } & \text { secondary electron imaging } \\ \text { STEM } & \text { scanning transmission electron microscope } \\ \text { TEM } & \text { transmission electron microscope }\end{array}$




\section{Abstract}

The unique combination of light weight, small dimensions, structural diversity, excellent mechanical strength and remarkable electronic properties make carbon nanotubes an attractive field of discovery for a wide range of applications, from reinforcing materials to molecular sensing. The immediate problem is in reliably and reproducibly fabricating carbon nanotubes and nanotube arrays with a certain exclusive structure. The reason for this is the large number of parameters integral to nanotube growth. This thesis describes the effect of several synthesis parameters - including temperature, catalyst, and water addition - on the growth of carbon nanotubes by a thermal chemical vapour deposition method. In all instances, multi-walled nanotubes were the only carbon nanotube products observed.

The chemical vapour deposition method employed here involves hexane as a volatile carbon precursor and ferrocene as a floating catalyst. The hexane is introduced into the system by passing a stream of nitrogen carrier gas through a bubbler containing the carbon precursor, while the ferrocene catalyst is positioned inside the working tube where it can evaporate gradually. The products of this method are large, vertically aligned arrays of clean multi-walled nanotubes. The second part of this thesis describes the role of the supporting layer in affecting the growth of these extended nanotube arrays.

A number of substrates have been examined - both conducting and non-conducting - and the products from these were analysed. It was found that all non-conductive, metal oxide substrates used - these included quartz, alumina, glazed porcelain, Pythagoras, and also fluorite - produced extended fields of carbon nanotubes. Conversely, many conductive substrates - including nickel, molybdenum, glassy carbon, highly ordered pyrolitic graphite and nickel-iron-silicon metal alloys - produce only small amounts of carbon 
nanotubes. This difference is likely caused by the deactivation of the iron catalyst at high temperature due to diffusion into the substrate surface. 


\section{Acknowledgements}

Firstly, I need to thank my supervisor Prof. John Spencer. His knowledge and guidance has been invaluable in the course of this thesis, both in the experimental and writing stages.

I need to thank Prof. Ken Mackenzie for his help in sourcing many of the substrates from IRL, and Dr. White for supplying them. Also, thanks to Drs. Ben Ruck and Andy Edgar for sourcing the rest of the substrates. Thanks also to Dr. John Abrahamson for graciously sending me high resolution electron microscopy images of CNTs from his original work into carbon arcs using graphite electrodes.

I would like to thank the University for funding me through the Tu Horomata Scholarship, as well as the School for finding me odd jobs to do to supplement this.

Thanks to my research group - and the rest of SCPS - for making this an easy environment to work in. In particular, I need to thank Kathryn Allan for proof-reading my work, which I know from experience is not easy to do.

That just leaves me to thank Nicole, my partner, and the rest of my family for supporting me during this period, especially when I was lacking in motivation and needed a push. 


\section{Table of Contents}

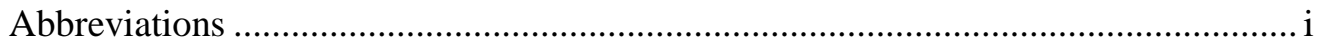

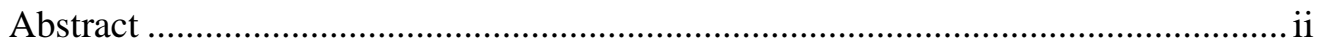

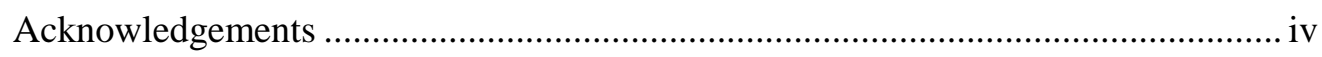

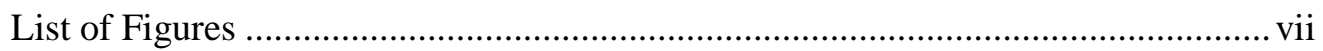

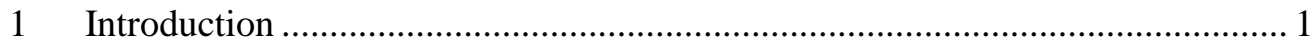

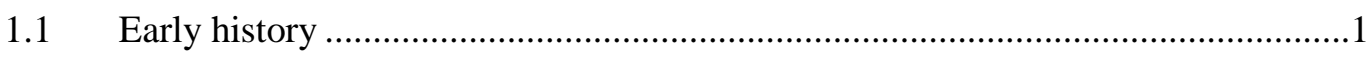

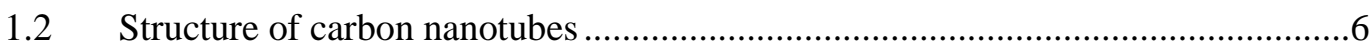

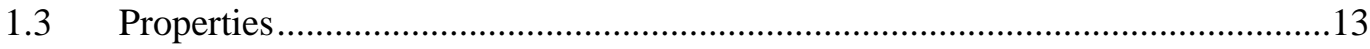

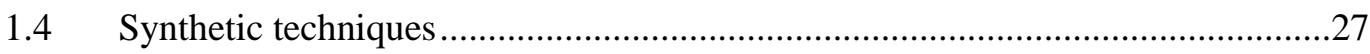

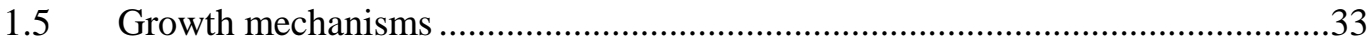

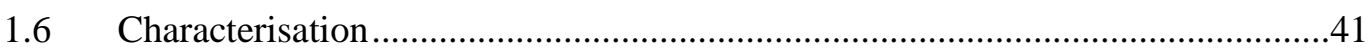

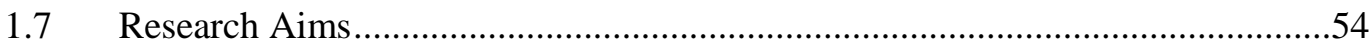

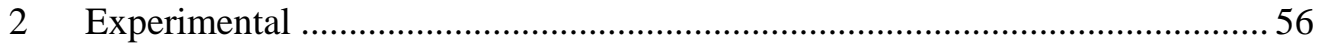

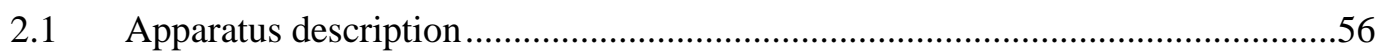

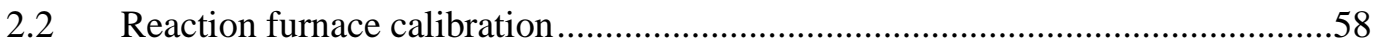

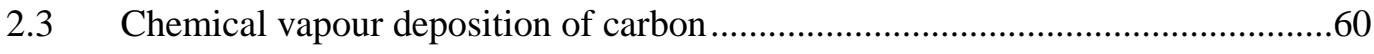

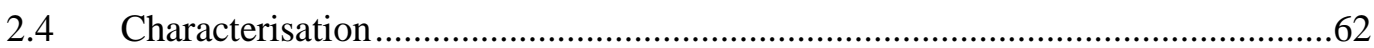

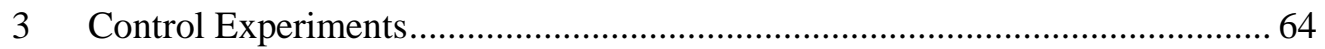

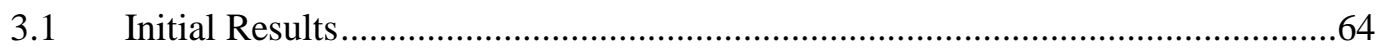

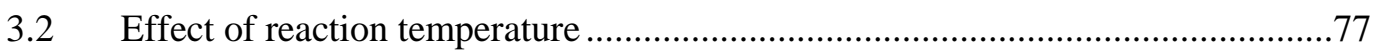

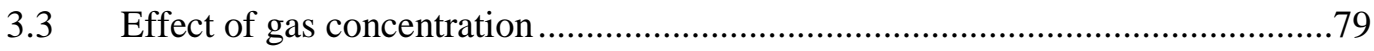

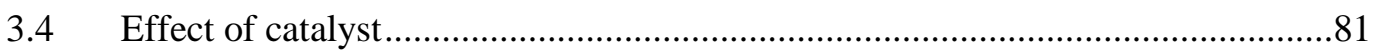

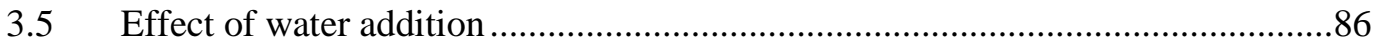

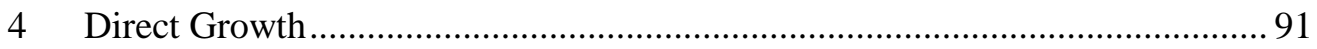




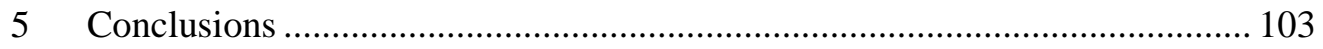

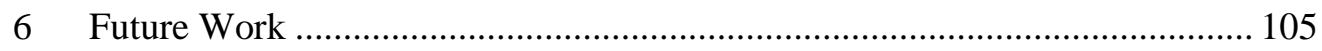

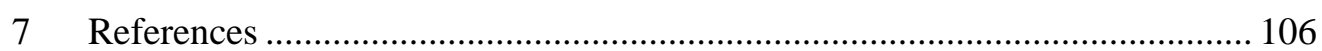




\section{List of Figures}

Figure 1-1: TEM image from the first discovery of carbon nanotubes published in 1952. Magnification: 20000x. ${ }^{2}$.

Figure 1-2: A) Secondary electron (SEI) and B) transmission electron microscope (TEM) images from the work of Wiles and Abrahamson, depicting carbon nanotube products after arc discharge across graphite electrodes. ${ }^{6}$

Figure 1-3: Finite structure of the planar allotrope of carbon, graphene. .7

Figure 1-4: Buckminsterfullerene, C60, (left) has a similar shape to the characteristic geodesic domes designed by Fuller, such as The Biosphère in Montréal, Canada (right). 8

Figure 1-5: Depiction of the layered Russian doll structure of a MWNT.

Figure 1-6: Schematic depicting the method for determining the chiral vector and vector indices for SWNTs.

Table 1: Summary of the vector indices $(n, m)$ and chiral angle $(\theta)$ for the three categories of SWNTs.

Figure 1-7: Three types of single-walled nanotubes with different helicities, assembled by rolling up a graphene sheet in different directions. Depicted are examples of A) an armchair $(6,6)$ tube; B) a chiral $(6,4)$ tube; $C)$ a zigzag $(6,0)$ tube. 12

Figure 1-8: TEM image depicting the increase in thermal vibrations with increasing temperature. ${ }^{18} 15$ Figure 1-9: Schematic of the method used by Salvetat et al. to measure the Young's modulus of SWNT ropes suspended across the pores of an ultrafitration membrane. ${ }^{21}$ 16

Figure 1-10: Wave-like distortion in the walls of large diameter MWNTs under flexural stress is an effective strain-relaxation mechanism leading to a noticeable decrease in the observed Young's modulus as diameter increases. ${ }^{23}$. 16

Figure 1-11: First Brillouin zone of graphene showing the allowed $k$-vectors of the $(8,0)$ nanotube. ${ }^{27} 18$ Figure 1-12: Energy dispersion relations of A) a metallic $(6,6)$ armchair tube and B) a semiconducting $(8,0)$ zigzag nanotube. ${ }^{30}$

Figure 1-13: Conductance vs. time of SWNT mats upon addition of A) nitrogen dioxide and B) ammonia. $^{34}$

Figure 1-14: A five inch, full colour, field emission display fabricated using SWNT tips as the field emitters. $^{39}$ 
Figure 1-15: Current vs. voltage plots of A) an individual SWNT displaying current saturation behaviour - the dashed line shows the Fowler-Nordheim fit - and B) a clean SWNT showing no current saturation, with the Fowler -Nordheim fit inset. ${ }^{42}$ 23

Figure 1-16: SEM image of A) a MWNT loop before high emitting current treatment and B) after treatment. $^{44}$ 24

Figure 1-17: Non-covalent $\pi-\pi$ stacking interactions between CNTs and pyrene derivatives. ${ }^{67} \ldots \ldots .26$

Figure 1-18: Controlled tethering of oxidized CNTs onto a gold surface using aminothiol linkers. ${ }^{67} 27$

Figure 1-19: Schematic for the apparatus and operation of the arc discharge method. ${ }^{69}$ 28

Figure 1-20: Schematic of the laser ablation apparatus. ${ }^{69}$ 30

Figure 1-21: Schematic of the reactor used by Smalley in the HiPCO process. ${ }^{73}$ 30

Figure 1-22: Simple schematic of a typical CVD set-up. ${ }^{78}$ 32

Figure 1-23: Binary phase diagram for the $\mathrm{Fe}-\mathrm{C}$ system. ${ }^{91}$ 35

Figure 1-24: Binary phase diagram of the $\mathrm{Fe}-\mathrm{C}$ system, focussing on the effect of catalyst particle size on the phase boundary between the solid and liquid phases and the associated eutectic point. ${ }^{92} 36$ Figure 1-25: Comparison of the models for the carbon dissolution and precipitation in a metal catalyst particle driven by A) a temperature gradient across the particle and B) a concentration gradient across the particle. ${ }^{95}$ 38

Figure 1-27: Model of the base and tip growth mechanisms. ${ }^{78}$ 40

Figure 1-28: Model of the 'scooter' mechanism. ${ }^{101}$ 41

Figure 1-29: Typical TEM image of a large diameter MWNT displaying the concentric lattice fringes. .44

Figure 1-30: Typical SEM image exhibiting large aligned fields of as-grown CNTs. A) is the image taken in SEI mode, while elemental maps of the same area, show the distribution of B) carbon and C) iron.

Figure 1-31: Sketch depicting the different forms of scattering when photons interact with molecules.

Figure 1-32: Raman active modes: A) The radial breathing mode and B) tangential stretching modes of the $\mathrm{G}$ band.

Figure 1-33: Comparative Raman spectra showing the tangential mode ( $\mathrm{G}$ band) region for highly ordered pyrolitic graphite (HOPG), a MWNT bundle, an isolated semiconducting SWNT and an 
isolated metallic SWNT. ${ }^{110}\left(\mathrm{G}^{+}\right.$refers to stretches along the tube axis while $\mathrm{G}^{-}$refers to circumferential stretches)

Figure 1-34: Plot of calculated transition energies, $E_{\mathrm{ii}}$, of all possible $(\mathrm{n}, \mathrm{m})$ combinations as a function of tube diameter, $d_{t}$ (Kataura plot). ${ }^{27}$ Superscript $M$ and $S$ are used to describe metallic and semiconducting CNTs, respectively. 54

Figure 2-1: Schematic depicting the experimental apparatus used for chemical vapour deposition of CNTs. .56

Figure 2-2: The purpose-built screw capped tapered glass fittings used to connect the working tube to the inlet and outlet gas flows. .57

Figure 2-3: Furnace calibration graph indicating the range of the independently measured temperature in a quartz tube under nitrogen flow for various furnace readings. .59

Figure 2-4: Reaction scheme for the preparation of cobaltocene.

Figure 3-1: SEM image comparing CNT deposits from A) the early region of the working tube (approx. 0-15 cm), before the hot zone and B) later in the tube $(>15 \mathrm{~cm})$, within the hot zone and beyond. 64

Figure 3-2: Comparative SEM images of the carbon deposits from different regions in the tube. A) SEI image of a deposit from early in the tube and B) the corresponding RBS image, while C) depicts an SEI image of a deposit from within the hot zone with D) its corresponding RBS image. 66 Figure 3-3: Comparative Raman spectra of CNT deposits from different regions of the working tube. Note the four low peaks in the $150-600 \mathrm{~cm}^{-1}$ region, evidence of iron oxide impurities in the Region B sample from earlier in the tube. 67

Figure 3-4: A) Low magnification SEM image of products from reactor sidewalls after CNT deposition. B) and C) are enlarged views of the indicated regions. 69

Figure 3-5: Cross-sectional view of an aligned field of CNTs. 70

Figure 3-6: A) High magnification SEM image of as-grown CNTs and B) a TEM image of an individual MWNT. C - F) STEM EDS maps depicting an iron particle within the walls of a MWNT. .72

Figure 3-7: Plot of TGA results of a pristine CNT array revealing two discrete regions of mass loss.73 
Figure 3-8: Comparative TEM images between A) an as-grown MWNT and B) after 30 min heating at $500{ }^{\circ} \mathrm{C}$ in air. There is a noticeable decrease in the amount of amorphous carbon. $\mathrm{C}-\mathrm{E}$ ) EDS maps illustrating the removal of carbonaceous material encapsulating the iron catalyst. 75

Figure 3-9: SEM image of a pristine, as-grown CNT field exhibiting a wave-like pattern of the CNTs. 76

Figure 3-10: A - C) Series of SEM images of increasing magnification, depicting a field of CNTs with two discrete growth domains. 77

Figure 3-11: Products from CNT deposition at A) $\left.700{ }^{\circ} \mathrm{C}, \mathrm{B}\right) 800^{\circ} \mathrm{C}$ and C) $900{ }^{\circ} \mathrm{C}$. 79

Figure 3-12: A) SEM image of a field of CNTs grown with an iron/carbon ratio of $15 \%$ and B) the corresponding RBS image with C) an EDS spectrum showing the number of counts of iron in the brighter top region (315 counts) versus D) the EDS spectrum showing the number of counts of iron in the lower darker region (180 counts).

Figure 3-13: Raman spectrum of the products from CNT deposition where the hexane is cooled to $-6^{\circ} \mathrm{C}$.

Figure 3-14: SEM images of MWNT products from the decomposition of cobaltocene. .82

Figure 3-15: SEM images of the products of CNT growth using a 10:1 iron/sulfur catalyst mix.....84 Figure 3-16: EDS images of small molybdenum and vanadium containing particles of various shapes and sizes.

Figure 3-17: $\mathrm{SEM}$ images of products from the CNT reaction using $\mathrm{Mo}_{72} \mathrm{~V}_{30}$ as a pre-deposited catalyst. A) Low magnification SEI image of apparently crystalline catalyst deposits with B) a high magnification image of a crystallite. C - E) EDS maps showing the distribution of carbon, molybdenum and vanadium respectively. 86

Figure 3-18: A) SEM image of a field of MWNTs and B) the corresponding RBS image illustrating the bright regions at the top of the MWNT field due to iron particles. C) SEM image of the interesting doughnut shaped CNT growth from water and D) the associated thermal analysis. .89 Figure 4-1: A) Low magnification SEM image of the top face of a region of quartz substrate after CNT deposition where the aligned field of CNTs has been scraped away during processing. B) Higher magnification image of a selected cross sectional region of a CNT array and C) of the top surface of a CNT array.

Figure 4-2: Raman spectrum of CNT products deposited on quartz. 93 
Figure 4-3: SEM images of CNT arrays grown on A) amorphous alumina, B) sapphire, C) Pythagoras, D) glazed porcelain and E) calcium fluoride. ...................................................... 94

Figure 4-4: SEM images of CNT deposition on A) $\beta$-sialon and B) O'-sialon...............................95

Figure 4-5: Interesting growth patterns on the surface of a Ni substrate....................................... 96

Figure 4-6: SEM images of CNT deposition on the surface of a molybdenum foil substrate...........98

Figure 4-7: SEM images of carbon deposits grown on the surface of A) HOPG, B) glassy carbon, C) MBF20 and D) MBF30. 99

Figure 4-8: Schematic representing the diffusion of catalyst into the substrate leading to catalyst deactivation. 101 


\section{Introduction}

The field of carbon nanotube (CNT) research has created an immense amount of interest amongst researchers and industry for the better part of the last two decades. The intense interest is a result of the wide range of remarkable physical, chemical and electronic properties that CNTs exhibit. These properties have provided a huge driving force in CNT research, to investigate firstly how to control nanotube growth, then to examine the observed properties of the nanotubes and finally to explore potential new applications for them. As such, CNT research is a highly multidisciplinary domain incorporating work from many different branches of science including fundamental physics, chemistry, materials science and engineering.

In this introduction a brief review of the interesting history relating to CNTs, particularly surrounding their early discoveries, will be given. The structure and classification of CNTs, and how these structures give nanotubes their extraordinary properties will be discussed. The different synthetic methods for growth of CNTs will be reviewed and the theories on the mechanism for nanotube growth explored. Finally, the methods of characterisation that are employed to investigate the structure and morphology of nanotubes and nanotube arrays will be examined.

\subsection{Early history}

The early history of CNTs is interesting as it dates back more than half a century and much of it is not widely known. In fact, in the last 12 to 24 months there has been a 
significant re-evaluation of this early record. While much of the scientific literature published before 2006, if not all, credits the first discovery of carbon nanotubes to Iijima for his seminal article published in Nature in $1991,{ }^{1}$ it is certainly not fair to do so. Indeed, if one looks back much further one can find published evidence of carbon nanotubes dating back to the 1950s. In 1952, two Soviet scientists, Radushkevich and Luk'yanovich, were investigating the thermal decomposition of carbon monoxide at $600{ }^{\circ} \mathrm{C}$ using iron catalysts, when the authors found what they described as 'carbon particles...of an elongated wormlike shape' (translated). ${ }^{2}$ One can see evidence of these elongated carbon particles in Figure 1-1.

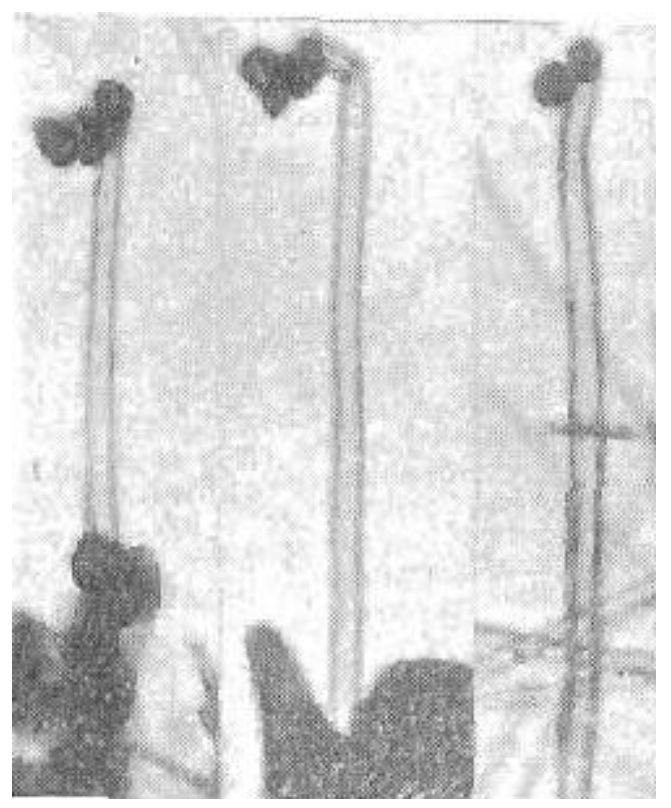

Figure 1-1: TEM image from the first discovery of carbon nanotubes published in 1952. Magnification: 20000x. ${ }^{2}$

Although they were not able to achieve the high resolutions and magnifications that are standard today, one can determine from the given magnification that these carbon tubes are $\sim 50 \mathrm{~nm}$ in diameter, and therefore are likely to be the first evidence of multi-walled carbon nanotubes (MWNTs). Moreover, this is not the only 
published example of carbon nanotubes that pre-dates Iijima's discovery. There are several further reports published during the 1970s and 1980s making reference to tubular carbon particles, ${ }^{3-5}$ including research from here in New Zealand. In 1978 Wiles and Abrahamson, from the University of Canterbury, published results from their investigations of low current carbon arcs using graphite electrodes and found nanotubes with diameters between 4 and $15 \mathrm{~nm} .{ }^{6}$ Hence, it is difficult to understand how none of these preceding published reports came to notice earlier.

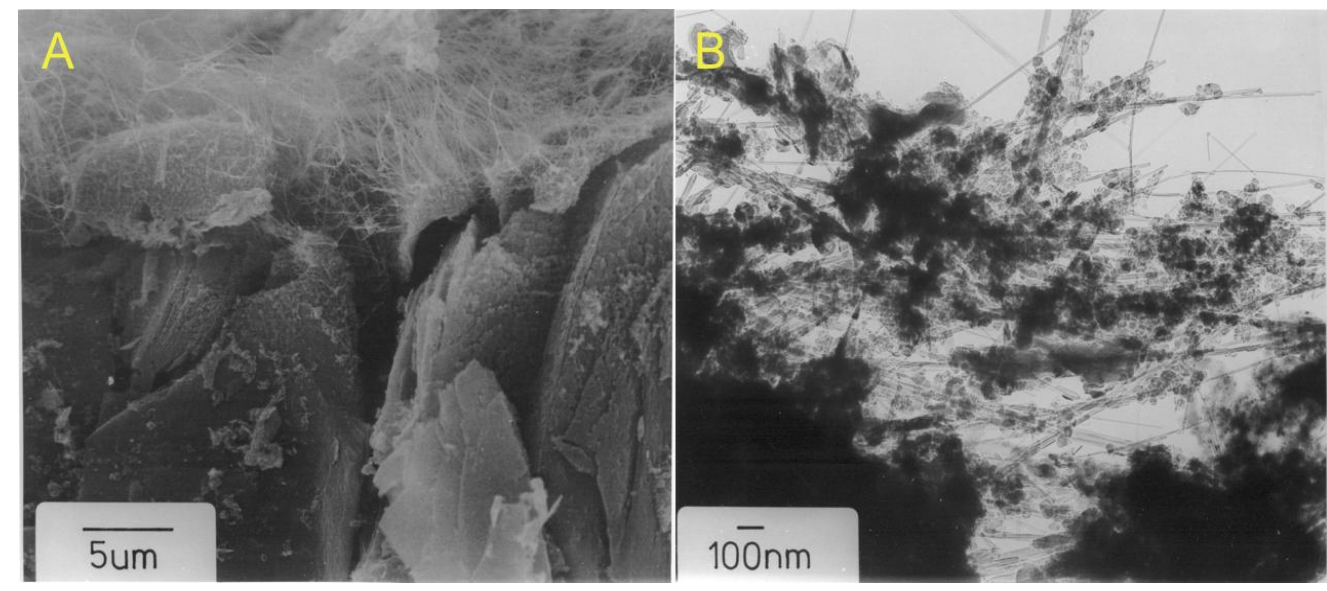

Figure 1-2: A) Secondary electron (SEI) and B) transmission electron microscope (TEM) images from the work of Wiles and Abrahamson, depicting carbon nanotube products after arc discharge across graphite electrodes. ${ }^{6}$

To best account for these oversights, two important factors perhaps need be considered: the timing of these publications and the publication source. In the case of the first published report, one needs to keep in mind that this was presented in Zhurnal Fizicheskoi Khimii (Russian Journal of Physical Chemistry) in 1952. ${ }^{2}$ Scientifically, at that time electron microscopy was still in its infancy, with the first microscopes only becoming commercially available in 1939. Consequently, electron microscopes would have been a very rare thing indeed, and those that were 
around would have rather limited resolution when compared to today's electron microscopes. This would have made investigation of CNTs very difficult in those early days. Beyond this, it was also a turbulent period politically. This report was presented during the Stalin era at the beginning of the Cold War and so Western scientists would have had very limited access to Soviet scientific literature at that time. Furthermore, the findings were published in the Russian language and could help to explain why this report managed to go largely unnoticed.

As for the reports from the 1970s and 1980s, these too were perhaps published before the scientific community was ready. While the idea of nanotechnology and its benefits had already been proposed by Richard Feynman in $1959,{ }^{7}$ and electron microscopy had progressed to a point where resolving nanometre-sized particles was not a great issue, the idea of miniaturisation had really only just sprouted, with the invention of the integrated chip in 1958. This made it possible to manufacture smaller and smaller devices, though it was only during the 1970s and 1980s that it began to be used in small home computers and other similar devices. Therefore, the community as a whole was conceivably still fixated by the fabrication of micrometre-sized devices for electronics.

Undoubtedly though, one of the pivotal points in the history of CNTs was the discovery of a new allotrope of carbon in 1985 by Smalley, Curl and Kroto - the fullerenes. ${ }^{8}$ Interested in the nature of carbon in interstellar matter, they began studying the laser vaporisation of a graphite target. From mass spectrometry of the products, the authors observed of a family of carbon clusters with different numbers of carbon atoms, and in particular one especially stable $\mathrm{C}_{60}$ cluster. The authors 
predicted that the stable cluster would be spherical in shape, much like a football, and named it buckminsterfullerene for its resemblance to the geodesic domes designed by the American architect, R. Buckminster Fuller. The family of related carbon clusters became known as the fullerenes and these changed the way scientists viewed carbon; until then it was thought that carbon had been well studied and so had nothing new to reveal. For their discovery, Smalley, Curl and Kroto were awarded the Nobel Prize in Chemistry in 1996, and this led to a renewed interest in the study of carbon.

The discovery of fullerenes paved the way for Iijima's 'rediscovery' in 1991 of carbon nanotubes, ${ }^{1}$ which are structurally related to the fullerenes. Interestingly, he referred to these as 'microtubules of graphitic carbon', even given their obvious nanometre dimensions. It now appeared the scientific community was ready for the discovery of this new form of carbon. While not the first published report on CNTs, nonetheless it was Iijima's article in the November issue of Nature that captured the imagination of researchers and propelled CNTs into the spotlight. One factor that almost certainly helped in this regard was the journal in which it was published. Nature is a highly respected journal within the scientific community, with an impact factor of 30.98, but most importantly it is read by people in a wide range of scientific disciplines. The earlier reports were often published in more specialised journals, so the exposure before this time was limited. Overall, this suggests that the impact or success of a particular report is not solely dependent on the strength of the findings, but sometimes also depends on the timing of publication and the journal in which it is published. 
However, while there is some conjecture over the initial discovery of CNTs, there is less ambiguity when it comes to the first discovery of single-walled CNTs. This is generally credited again to Iijima, ${ }^{9}$ but in conjunction with a group from IBM headed by Bethune. ${ }^{10}$ These two groups independently published reports in the same issue of Nature, in April 1993, describing CNTs made from a single graphene sheet.

Since these early beginnings there has been a veritable explosion in the amount of published research within the multidisciplinary field of carbon nanotubes, so that today it represents one of the most active fields of the natural sciences with more than 100 new reports published weekly.

\subsection{Structure of carbon nanotubes}

While the considerable interest in carbon nanotube research is a product of the many fascinating properties they exhibit, these properties are a direct result of the diverse structures possible for carbon nanotubes. Thus it is important to examine the different nanotube forms in some detail. In order to understand carbon nanotube structure it is useful to first consider the structure of two related forms of carbon graphene and buckminsterfullerene. As depicted in Figure 1-3, graphene is a planar sheet composed of trivalent, $\mathrm{sp}^{2}$-bonded carbon atoms that form a perfect twodimensional hexagonal array. The $\mathrm{sp}^{2}$-bonded nature of the carbon atoms allows for the delocalisation of $\pi$-electrons throughout the sheet, giving the structure aromaticity. It is these graphene sheets that, when stacked, constitute graphite.

\footnotetext{
* Estimate based on number of reports found during surveys of academic literature using Chemical Abstracts Service carried out in May 2007 (38000+) and again in May 2008 (48000+), in conjunction with regular surveys during November/December 2007.
} 


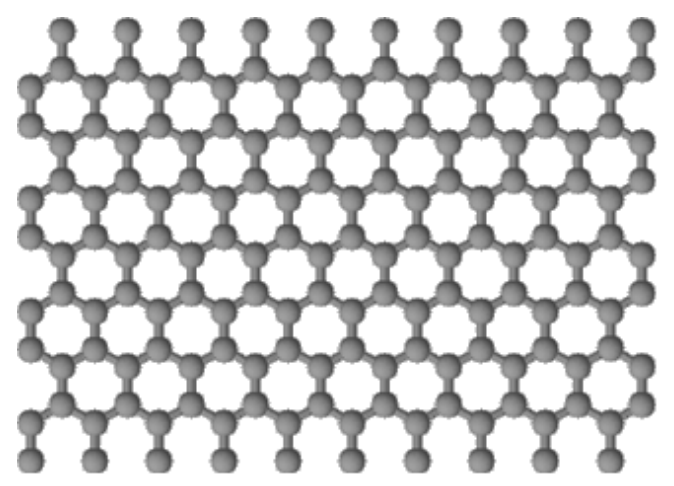

Figure 1-3: Finite structure of the planar allotrope of carbon, graphene.

Importantly, the introduction of structural defects within the graphene layer can disturb this network, causing a distortion of the planar structure. For instance, the inclusion of heptagonal rings into the hexagonal structure causes the sheet to fold into a saddle shape. Likewise, the inclusion of one pentagonal ring into the structure causes the sheet to fold into a cone, while the insertion of 12 pentagonal rings causes the graphene sheet to completely fold around onto itself to form a cage, as for the fullerenes. Buckminsterfullerene, $\mathrm{C}_{60}$, is the most stable of this family of fullerenes and can be imagined as the result of folding an imperfect graphene sheet to form a truncated icosahedron. Depicted in Figure 1-4, it contains 20 hexagonal rings and 12 pentagonal rings and is the smallest fullerene wherein no two pentagons share an edge, thought to be the reason for its exceptional stability. ${ }^{11}$ 

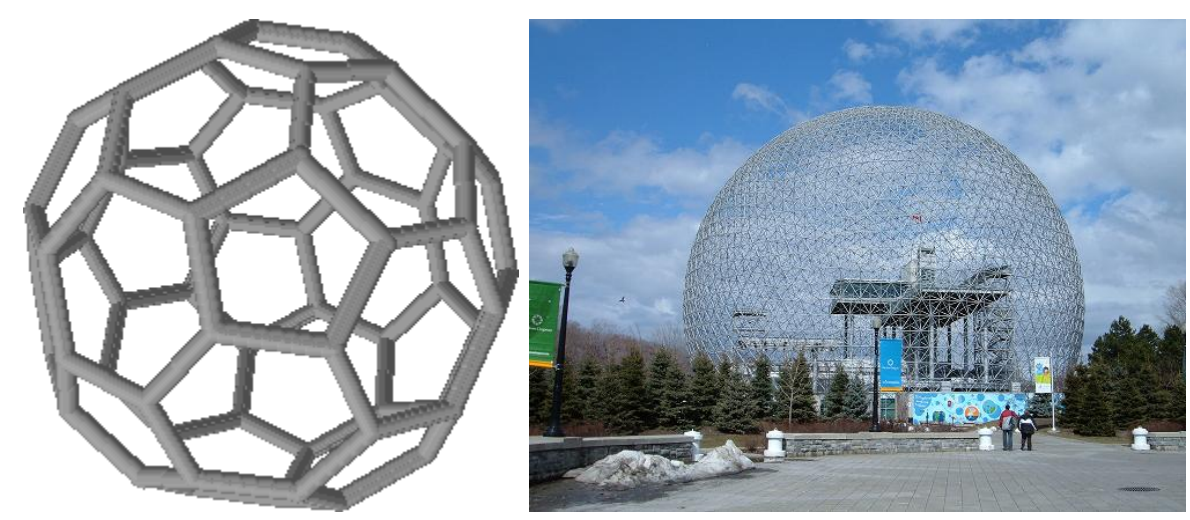

Figure 1-4: Buckminsterfullerene, C60, (left) has a similar shape to the characteristic geodesic domes designed by Fuller, such as The Biosphère in Montréal, Canada (right).

Carbon nanotubes are a cylindrical form of carbon related to the fullerenes, with high length to diameter ratios. Fundamentally, carbon nanotube structures can be grouped into two broad classes: single-walled nanotubes (SWNTs) and multi-walled nanotubes (MWNTs). While there are several different forms of carbon nanotube structure within these classes, to understand their structure it is simplest to begin with an idealised carbon nanotube. Generally, an idealised carbon nanotube is shaped like a cylinder with a convex hemispherical cap at both ends. In simple terms, one can imagine constructing this shape by cutting a molecule of $\mathrm{C}_{60}$ in half, then forming a cylinder by rolling up a single graphene sheet and implanting it between the two hemispherical caps. The resulting closed tube is a single-walled nanotube.

Multi-walled nanotubes are a variation of this structure where several tubes are found within one another, in a Russian doll-like arrangement, as in Figure 1-5. As the number of walls can vary greatly, from only a few to hundreds, so too do the diameters for MWNTs, covering a wide range up to $100 \mathrm{~nm}$. Generally, the spacing between subsequent walls is found to be relatively regular, at $0.344 \mathrm{~nm}$ for tubes 
greater than $10 \mathrm{~nm} .{ }^{1}$ However, for diameters smaller than this, there tends to be a higher curvature of the individual tubes leading to an increase in the layer spacing up to approximately $0.4 \mathrm{~nm} .{ }^{12}$ Furthermore, while an ideal SWNT can be considered as a rolled sheet of graphene with hemispherical caps, both types of nanotube structure can be found with other shaped caps or with open ends. ${ }^{13}$
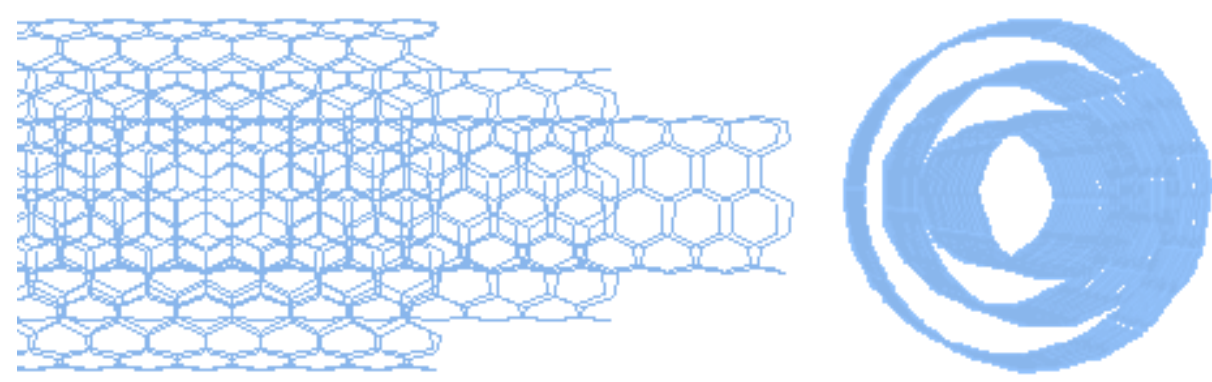

Figure 1-5: Depiction of the layered Russian doll structure of a MWNT.

If one disregards MWNTs for a moment and focuses on SWNTs instead, one finds there are many possible structures that SWNTs can adopt. When one considers constructing a SWNT from a flat graphene sheet, the sheet can be wrapped in many different ways. For instance, one can cut a strip from a graphene sheet in any direction, and when rolled up, the strip will form a seamless tube composed solely of hexagons. However, strips cut in different directions will cause the position of the hexagons around the circumference to differ when wrapped up. This is observed as a different "twist" of the graphene sheet, termed the helicity or chirality of a tube. 

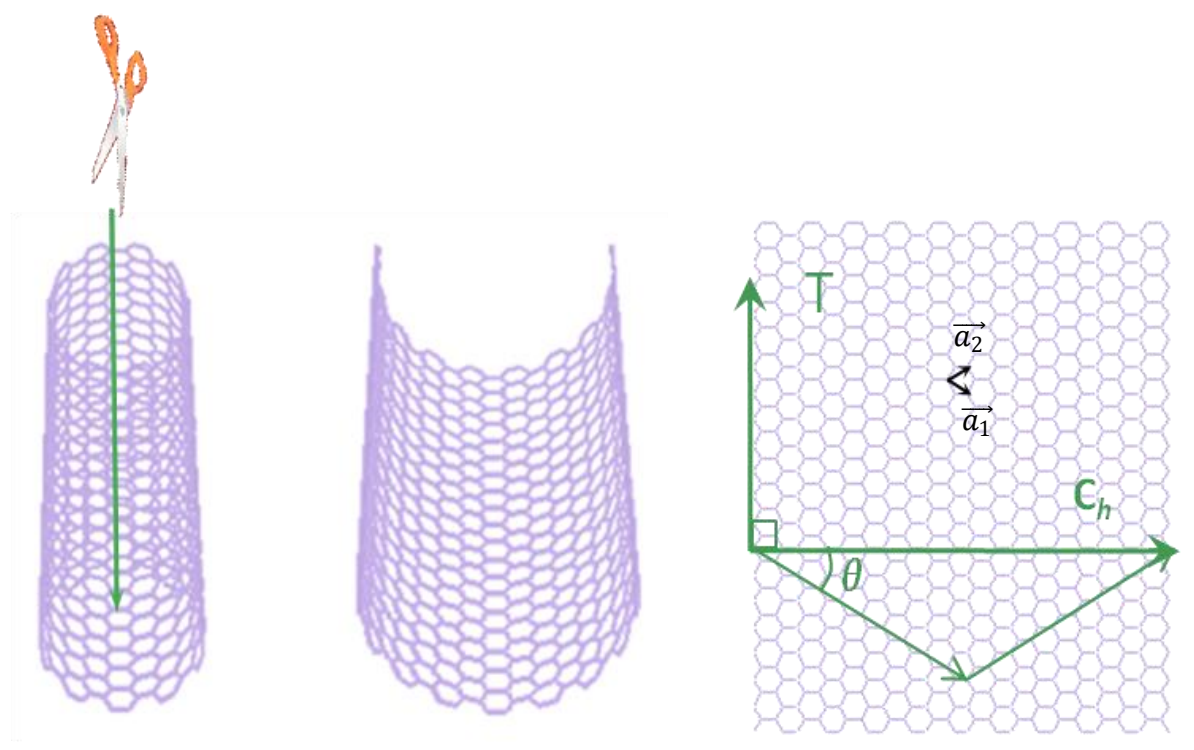

Figure 1-6: Schematic depicting the method for determining the chiral vector and vector indices for SWNTs.

In order to differentiate between tubes with different helicities, a system is used to describe nanotubes based on the vector indices of their constituent graphene network. Firstly, one needs to imagine opening a nanotube by slicing along a tube wall, parallel to the tube axis (T), and then unfolding the nanotube to form a flat graphene sheet, as shown in Figure 1-6. The chiral vector $\left(\mathrm{C}_{h}\right)$, by convention, is defined as the vector perpendicular to $\mathrm{T}$, and spans the full width of the graphene sheet between two atom sites, which are crystallographically equivalent, corresponding to the full circumference of the rolled tube. The chiral vector can be further described as the vector sum of two unit vectors, $\overrightarrow{a_{1}}$ and $\overrightarrow{a_{2}}$ in Figure 1-6. This relationship is given in Equation 1-1:

$$
C_{h}=n \overrightarrow{a_{1}}+m \overrightarrow{a_{2}}
$$

where $n$ and $m$ are vector coefficients and $0 \leq|m| \leq n$. 
By counting the number of integer unit vector steps across the graphene lattice, simply the number of hexagons in each direction, one reveals the vector coefficients $n$ and $m$, referred to as the vector indices. From here, one can also determine the chiral angle $(\theta)$, defined as the angle between the chiral vector and the unit vector $\overrightarrow{a_{1}}$. The chiral vector, and consequently the vector indices, dictates the chiral angle and ultimately the diameter of a given tube. Therefore, quoting a nanotube in terms of its vector indices $(n, m)$ provides a convenient method to describe distinct SWNTs. The relationship between the vector indices and the tube diameter is given by Equation 1-2:

$$
d_{t}=\frac{a_{C C} \cdot \sqrt{3\left(n^{2}+n m+m^{2}\right)}}{\pi}
$$

where $d_{t}$ is the tube diameter and $a_{C C}$ is the C-C bond length in bulk graphite $(1.42 \AA)$.

The many helical variations of SWNTs can be classified into three broad categories: armchair, zigzag and chiral. When a tube's vector indices are the same, i.e. $n=m$, it exhibits an armchair structure and the chiral angle is $30^{\circ}$. Conversely, if a tube has either $n$ or $m$ equal to 0 , then it exhibits a zigzag structure, where the chiral vector runs along the zigzag bonds of the lattice and the tube is considered to have a chiral angle of $0^{\circ}$. Finally, for all other integer values of $n$ and $m-$ i.e. $n \neq m$ and $n, m \neq 0-$ the tube is considered chiral, and the chiral angle will be between 0 and $30^{\circ}$. Examples of each type of tube are given in Figure 1-7, while their vector indices and chiral angles are summarised in Table 1. 
Table 1: Summary of the vector indices $(n, m)$ and chiral angle $(\theta)$ for the three categories of SWNTs.

\begin{tabular}{ccc}
\hline Type & $(\boldsymbol{n}, \boldsymbol{m})$ & $\boldsymbol{\theta}$ \\
\hline Armchair & $n=m$ & $30^{\circ}$ \\
Zigzag & $n$ or $m=0$ & $0^{\circ}$ \\
& Any integer where & $0<\theta<30^{\circ}$ \\
Chiral & $n \neq m$ and $n, m \neq 0$ & \\
\hline
\end{tabular}

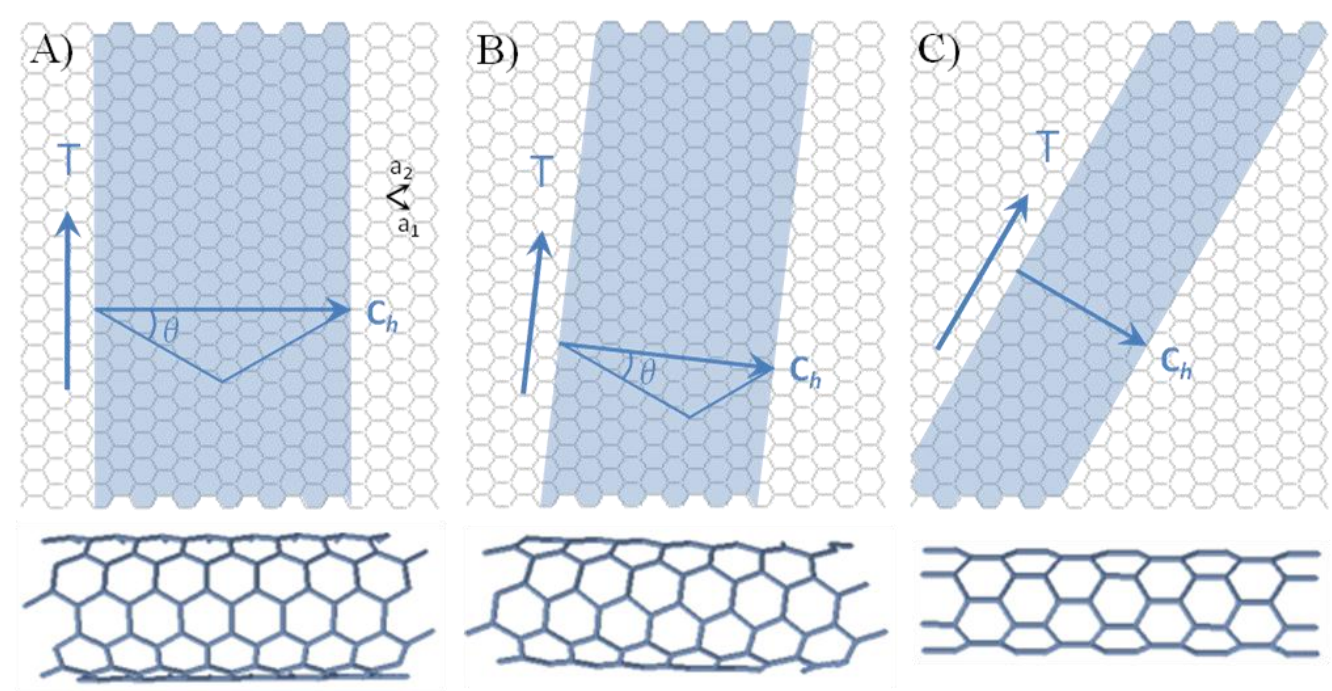

Figure 1-7: Three types of single-walled nanotubes with different helicities, assembled by rolling up a graphene sheet in different directions. Depicted are examples of A) an armchair $(6,6)$ tube; B) a chiral $(6,4)$ tube; C) a zigzag $(6,0)$ tube.

For MWNTs, in theory the individual constituent layers can adopt any one of these different helicities and so there are any numbers of variations possible over the whole structure. The difficulty is in the determination of the chirality for each individual layer, as the normal technique of chirality determination of SWNTs by analysis of the radial breathing modes in the Raman spectrum is not appropriate for 
MWNTs. ${ }^{\dagger}$ However, in a report by Hassanien et al. the authors were able to scan an image of the outer three layers of a MWNT using scanning tunnelling microscopy (STM) and spectroscopy (STS), though they were unable to resolve the atomic arrangement of the second and third layers due to some amorphous carbon impurities. ${ }^{12}$ This indicates that in the future, STM may be one tool that is able to probe the chirality of several layers in a single MWNT.

\subsection{Properties}

Though their small size and wide array of structures make CNTs an interesting subject, it is the properties that are exhibited as a result of nanotube structure that are most interesting. The distinctive combination of mechanical, electrical, thermal and chemical properties makes CNTs a most attractive material for study.

\section{Mechanical properties}

The strong graphitic framework that comprises the nanotube body confers upon it remarkable mechanical features. There have been many theoretical predictions of the mechanics, while several different groups have endeavoured to quantify some of these properties, particularly the modulus of elasticity (Young's modulus) and the tensile strength. Yakobson et al. calculated a Young's modulus of 5 TPa when using many-body interatomic potentials to carry out molecular dynamics simulations. ${ }^{14}$ This number is exceedingly high and is, in fact, five times higher than for graphite, at $1.06 \mathrm{TPa}^{15}$ Using an empirical force-constant model, Lu calculated the bulk, shear and Young's moduli of SWNTs and MWNTs over a range of diameters, helicities and number of walls. ${ }^{16}$ The author found a value of 1 TPa for the Young's

\footnotetext{
${ }^{\dagger}$ For further discussion of radial breathing modes and their characterisation, see pg 44.
} 
modulus of CNTs, in accordance with bulk graphite, which was largely independent of the structural parameters for tubes between 0.3 and $13 \mathrm{~nm}$ in diameter. Meanwhile, using a tight binding method, Hernandez and associates were able to calculate a value of $1.26 \mathrm{TPa}$ for the Young's modulus of SWNTs. ${ }^{17}$ Again, this is in good agreement with the value for graphite.

The first experimental measurement of the Young's modulus was performed by Treacy and co-workers in $1996 .{ }^{18}$ Illustrated in Figure 1-8, by measuring the frequency of thermal vibrations of 11 individual MWNTs over a range of temperatures using TEM, the authors were able to find an average value of $1.8 \mathrm{TPa}$, although the actual values measured ranged from $0.4 \mathrm{TPa}$ to a maximum of 4.15 TPa. This is similar to that measured for graphite and confirmed the earlier predictions of high mechanical strength. In a later study undertaken by the same group, the mean Young's modulus of SWNTs was found to be $1.25 \mathrm{TPa}$, though on this occasion using a much larger test sample population (27 tubes) to increase sampling accuracy. ${ }^{19}$ 


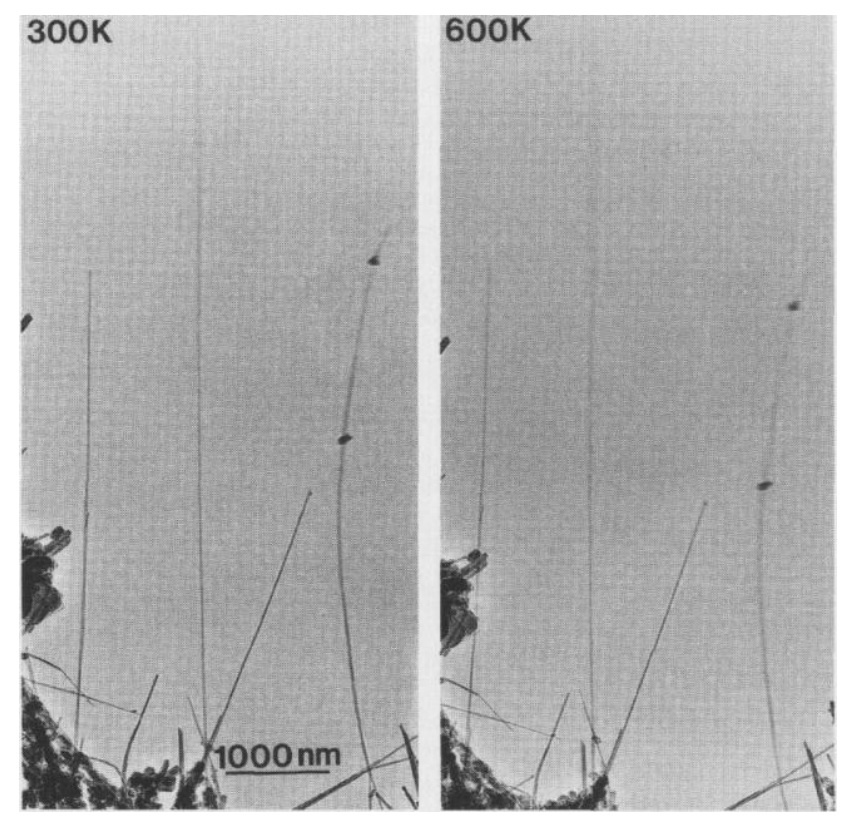

Figure 1-8: TEM image depicting the increase in thermal vibrations with increasing temperature. ${ }^{18}$

However, Wong et al. employed a different technique to measure the Young's modulus of MWNTs. ${ }^{20}$ These authors used an atomic force microscope (AFM) tip to bend cantilevered tubes and simultaneously measured the force exerted by the tubes as a function of displacement. From this information, a Young's modulus of 1.28 $\mathrm{TPa}$ was determined. Salvetat and colleagues used yet another approach, depicted in Figure 1-9, instead suspending CNTs across the pores of a polished alumina ultrafiltration membrane and pressing on the suspended section with an AFM tip. ${ }^{21,22}$ By measuring both the applied force and subsequent deflection, the authors were able to determine an average Young's modulus of 1 TPa for SWNT ropes $^{21}$ and $0.81 \mathrm{TPa}$ for arc discharge-grown MWNTs, whereas catalytically-grown MWNTs exhibit a much lower elastic modulus of between 0.01 and $0.05 \mathrm{TPa}^{22}$ The much lower value for catalytically-grown MWNTs is due to the significantly higher occurrence of defects compared with those grown from arc discharge and clearly demonstrates the impact of point defects in the CNT structure on the observed properties. 


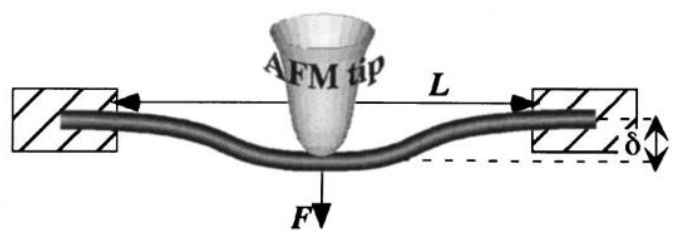

Figure 1-9: Schematic of the method used by Salvetat et al. to measure the Young's modulus of SWNT ropes suspended across the pores of an ultrafitration membrane. ${ }^{21}$

Poncharal and associates used a different technique to measure the elastic modulus, instead measuring the deflections of MWNTs when subjected to static and oscillating potential fields. ${ }^{23}$ The authors found that for small tubes $\left(d_{t}<12 \mathrm{~nm}\right)$ the Young's modulus was $\sim 1 \mathrm{TPa}$, however this decreases significantly to $\sim 0.1 \mathrm{TPa}$ for CNTs with $d_{t}=40 \mathrm{~nm}$. This sharp drop was attributed to a wave-like distortion of the walls on the side under compression, as depicted in Figure 1-10, which acts as an effective strain-relaxation mechanism.
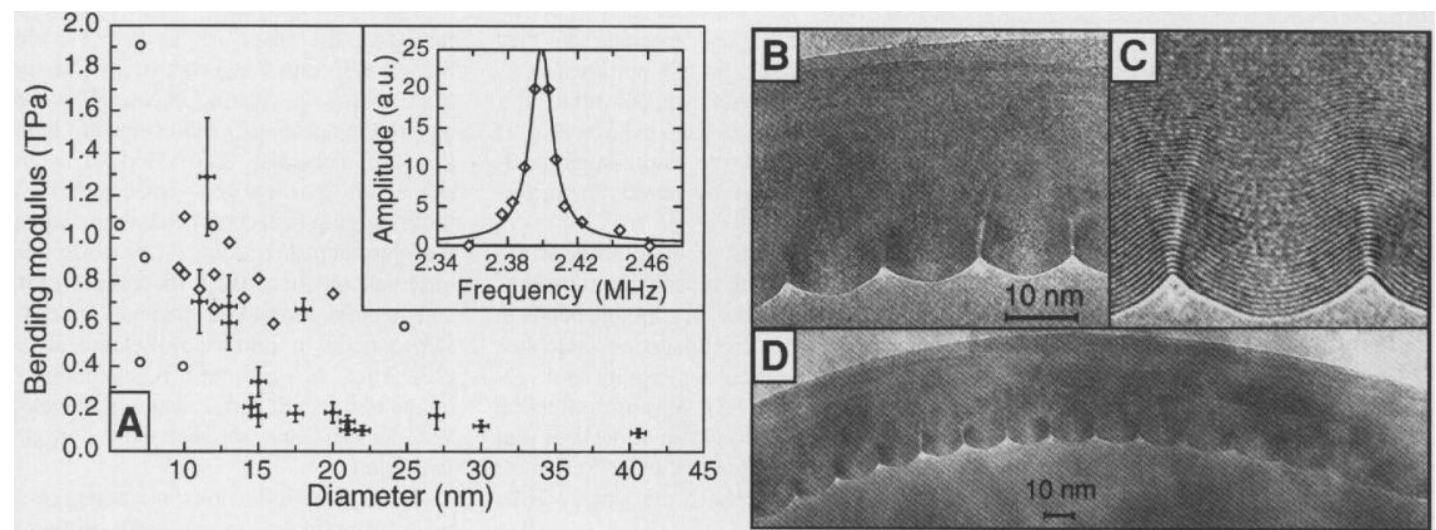

Figure 1-10: Wave-like distortion in the walls of large diameter MWNTs under flexural stress is an effective strain-relaxation mechanism leading to a noticeable decrease in the observed Young's modulus as diameter increases. ${ }^{23}$ 
These experiments have shown that CNTs show remarkable stiffness when bent, however the strong graphite network also imparts high strength under tensile loads. Wagner et al. used a common method of measuring the strength of fibres by embedding MWNTs into a polymer matrix and then subjecting the composite to strain. The authors found tensile strengths of $\sim 55 \mathrm{GPa}$ and a Young's modulus of 1.8 $\mathrm{TPa}$ for these embedded nanotubes. Alternatively, $\mathrm{Yu}$ and associates used an AFM tip to pull the ends of a nanotube to measure the tensile strengths of both $\mathrm{SWNTs}^{24}$ and MWNTs, ${ }^{25}$ ranging $13-52 \mathrm{GPa}($ mean $=30 \mathrm{GPa})$ and $11-63 \mathrm{GPa}$ (no mean value quoted) respectively.

Thus, when compared to other common high strength materials such as steel and Kevlar® in Table 2, it is apparent that the mechanical properties of CNTs are far superior. However, particular emphasis must be placed on the fact that these high strength features are coupled with a very low density, making CNTs a very attractive structural reinforcing material.

Table 2: Comparison of mechanical properties of a range of materials.

\begin{tabular}{cccc}
\hline Material & $\begin{array}{c}\text { Density } \\
\left(\mathbf{g ~ c m}^{-3}\right)\end{array}$ & $\begin{array}{c}\text { Young's Modulus } \\
\text { (TPa) }\end{array}$ & $\begin{array}{c}\text { Tensile Strength } \\
(\mathbf{G P a})\end{array}$ \\
\hline SWNT & $\sim 1.40$ & $\sim 1.25$ & $\sim 30$ \\
MWNT & $\sim 1.40$ & $\sim 1.28$ & $\sim 50$ \\
graphite $^{\mathbf{1 3}}$ & 2.26 & 1.06 & 13 \\
steel & 7.86 & 0.21 & 0.46 \\
Kevlar $^{\circledR 26}$ & 1.47 & 0.18 & 3.4 \\
\hline
\end{tabular}




\section{Electronic properties}

One of the most advantageous aspects of CNTs is their peculiar electronic properties. These properties are a result of their small size and high aspect ratio, causing confinement of the propagation of electrons to one dimension. To understand the electronic properties, it is useful to first examine the electronic structure of CNTs. As an approximation, it is easier to look at the Brillouin zone of graphene. The Brillouin zone is the volume of the primitive cell of the reciprocal lattice, so for graphene it appears hexagonal, as shown in Figure 1-11.

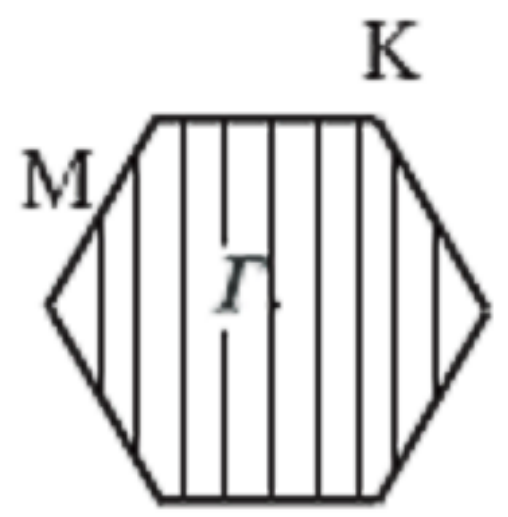

Figure 1-11: First Brillouin zone of graphene showing the allowed $k$-vectors of the $(8,0)$ nanotube. ${ }^{27}$

Early work by Hamada et al. and Saito et al. employed a tight-binding model to calculate the energy bands of different nanotubes based on a zone folding technique of the Brillouin zone of graphene. ${ }^{28,29}$ The cylindrical geometry of a nanotube imposes a periodic boundary condition around the circumference on a propagating electron, thus only certain wave lines - or $k$-vectors - are allowed. These allowed $k$ vectors give rise to the unique band structure of nanotubes, depicted in Figure 1-12, which are dependent on both the diameter and the chirality of the tube. ${ }^{28}$ 

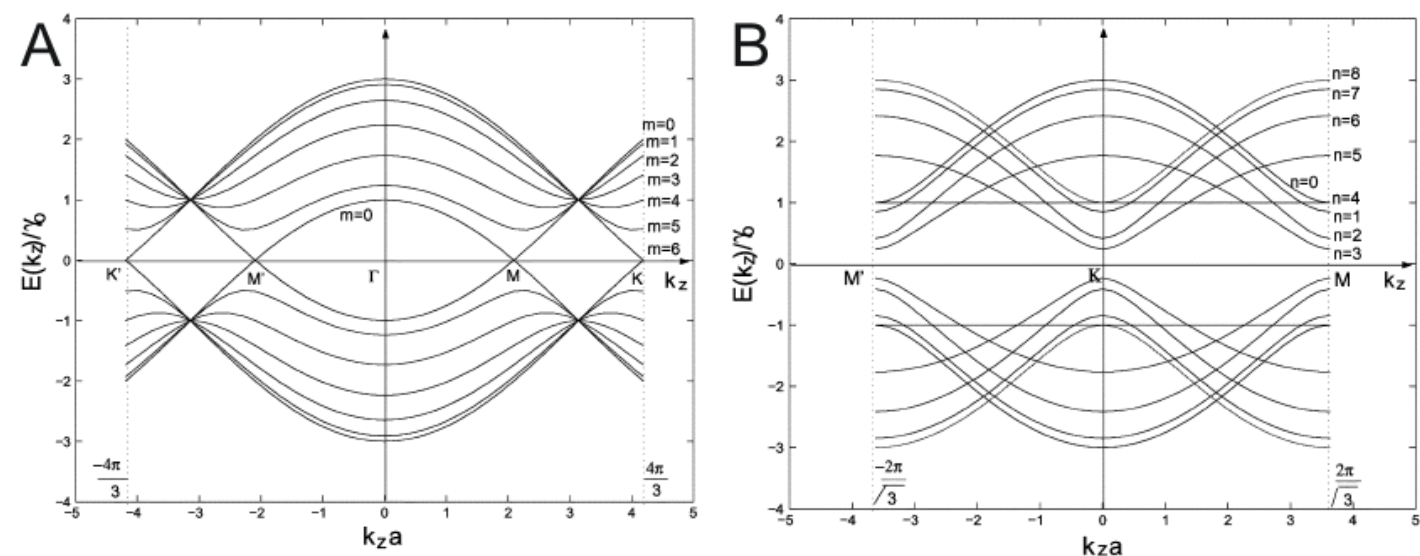

Figure 1-12: Energy dispersion relations of A) a metallic (6,6) armchair tube and B) a semiconducting $(8,0)$ zigzag nanotube. ${ }^{30}$

For a SWNT with real space vector indices $(n, m)$, when $n-m=0$, as is the case for armchair nanotubes, the resulting tube will exhibit metallic behaviour. In addition, where $n-m=3 l$ and where $l$ is a non-zero integer, the tube will also behave like a metallic conductor. For all other SWNTs - i.e. when $n-m$ is not divisible by three - they will behave as large gap semiconductors. Consequently, $\frac{2}{3}$ of all SWNTs are semiconducting and $\frac{1}{3}$ are metallic. The actual band gap is inversely dependent on the diameter of the tube, so that as diameter increases the band gap tends to zero. ${ }^{28}$

However, the tight-binding model does not take into account the curvature effects due to the cylindrical nature of CNTs. Blase and co-workers employed ab initio pseudopotential local density functional calculations to analyse these curvature effects. $^{31}$ They demonstrated that for SWNTs with small diameters, the high curvature of the wall causes the hybridisation of the $\sigma^{*}$ and $\pi^{*}$ orbitals. This leads 
to a decrease in the observed band gap. Conversely, Kane and Mele showed that while tube curvature does not affect armchair nanotube metallicity, for nanotubes where $n-m=3 l$ tube curvature opens up a tiny band gap, although this gap is so small that at room temperature it is essentially metallic. ${ }^{32}$ Furthermore, Tang and colleagues found that nanotubes with $0.4 \mathrm{~nm}$ diameter display 1D superconductivity below $15 \mathrm{~K}^{33}$

Some issues arose when trying to confirm these predictions of the intrinsic nature of SWNT conduction when experimental reports began to show variable conduction behaviour in different environments. Kong et al. found that nitrogen dioxide caused an increase in the conductance of SWNTs, while ammonia caused a decrease in the conductance, as demonstrated in Figure 1-13. ${ }^{34}$ This is due to doping of the nanotubes, where the presence of nitrogen dioxide molecules brings the conduction band closer to the Fermi level, thus increasing the number of charge carriers, whereas ammonia shifts the valence band further from the Fermi level. A similar effect was found with oxygen, causing an increase in the conduction of SWNTs. ${ }^{35}$
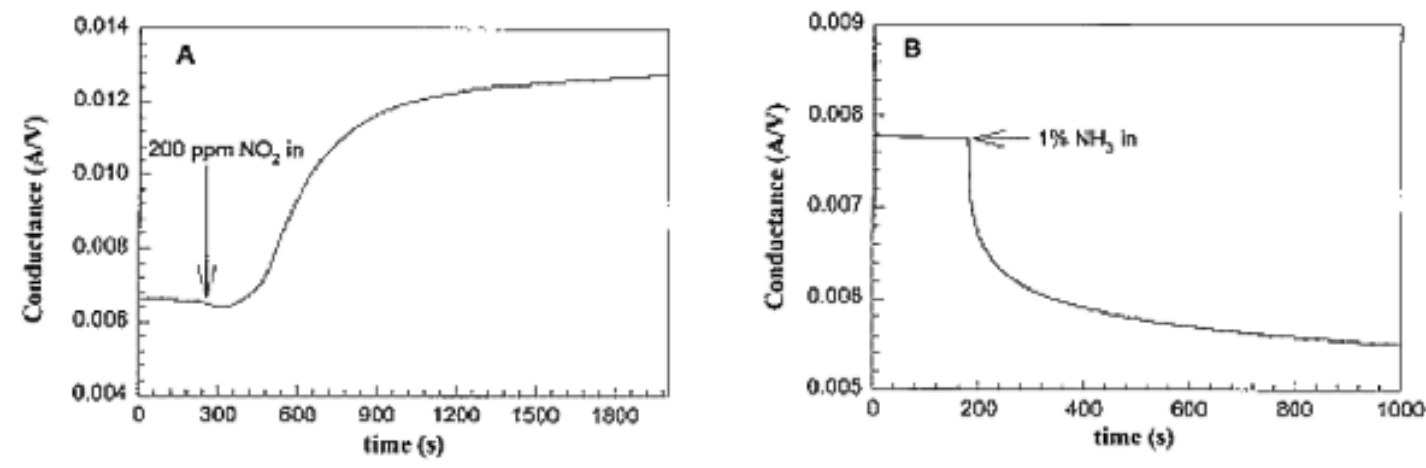

Figure 1-13: Conductance vs. time of SWNT mats upon addition of A) nitrogen dioxide and B) ammonia. $^{34}$ 
The different electronic properties of SWNTs inspire interest in the electronic behaviour of MWNTs, where the combination of multiple coaxial tubes could provide interesting results. Saito et al. showed that for double walled nanotubes, each wall retains its conduction character, with only very weak interlayer coupling. ${ }^{36}$ This suggests that metal-semiconductor junctions within a MWNT are possible. However, for many MWNTs their conduction behaviour is limited by their large diameters, and hence exhibit no band gap as described above.

While the conductive behaviour of CNTs is very interesting, so too, is their transport behaviour. Frank et al. found that the conductance in metallic MWNTs at room temperature is quantised. ${ }^{37}$ This leads to the property of ballistic transport - the conductance of electrons along the tube length without scattering. In addition, the authors found that these metallic MWNTs could sustain stable current densities in excess of $10^{7} \mathrm{~A} \mathrm{~cm}^{-2}$, a $10^{5}$ increase over copper. Further work from the same group determined a mean free path of $60 \mu \mathrm{m}$ for the transport of electrons within CNTs. ${ }^{38}$

One final electrical property that makes CNTs particularly remarkable is their field emission capability. Field emission is the tunnelling of electrons from the surface of a material in the presence of an external field. This phenomenon is useful in display technology. Due to their high aspect ratios and high current densities, CNT arrays already show promise for the manufacture of new flat panel field emission displays (Figure 1-14)..$^{39}$ 


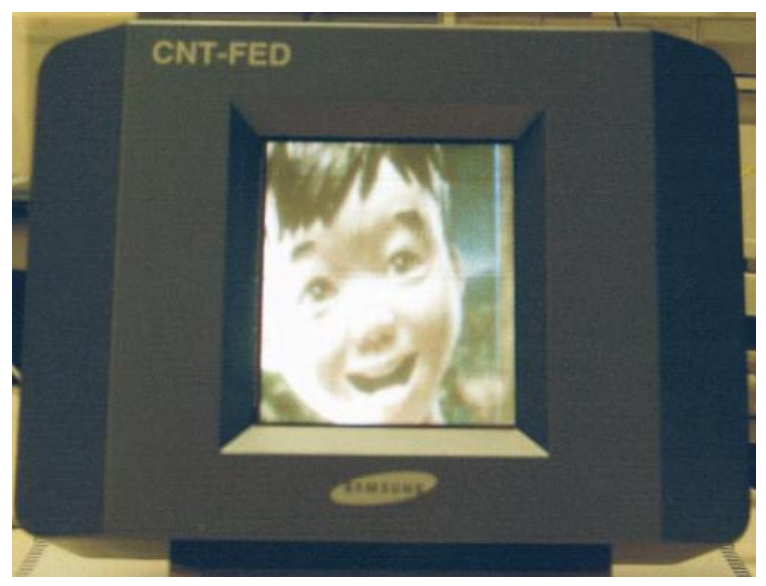

Figure 1-14: A five inch, full colour, field emission display fabricated using SWNT tips as the field emitters. ${ }^{39}$

It is widely assumed that the field emission behaviour of CNTs follows that of metals, due to the frequent metallic or semimetallic behaviour of CNTs. This means that CNTs should follow Fowler-Nordheim theory, which quantitatively describes the current density of field emission of metals as a function of field strength. CNTs have been shown to emit electrons in this way, but a saturation of the current emission is often observed at high fields. Several groups have tried to explain this limiting current behaviour. Yaghoobi and Nojeh suggest that CNTs differ from the Fowler-Nordheim model as the model assumes the emitting surface is planar, which is certainly not true for CNTs, so cannot be suitably applied for CNTs. ${ }^{40}$ Zheng and co-workers propose that the geometry at the nanotube tip means a potential well could exist at the tip, thus greatly affecting the observed field emission. ${ }^{41}$

Alternatively, Dean and Chalamala suggest that this current saturation behaviour is caused by adsorbates. $^{42}$ Initially, the authors found the same current saturation effect when measuring the field emission of individual SWNTs, but after removing all adsorbates by heating above $900 \mathrm{~K}$ under ultra-high vacuum, they discovered that 
the current limiting effect was removed, as depicted in Figure 1-15. Furthermore, when the authors introduced clean SWNTs to water vapour they found that the conductance increased by two orders of magnitude while the current saturation effect reappeared at high field.
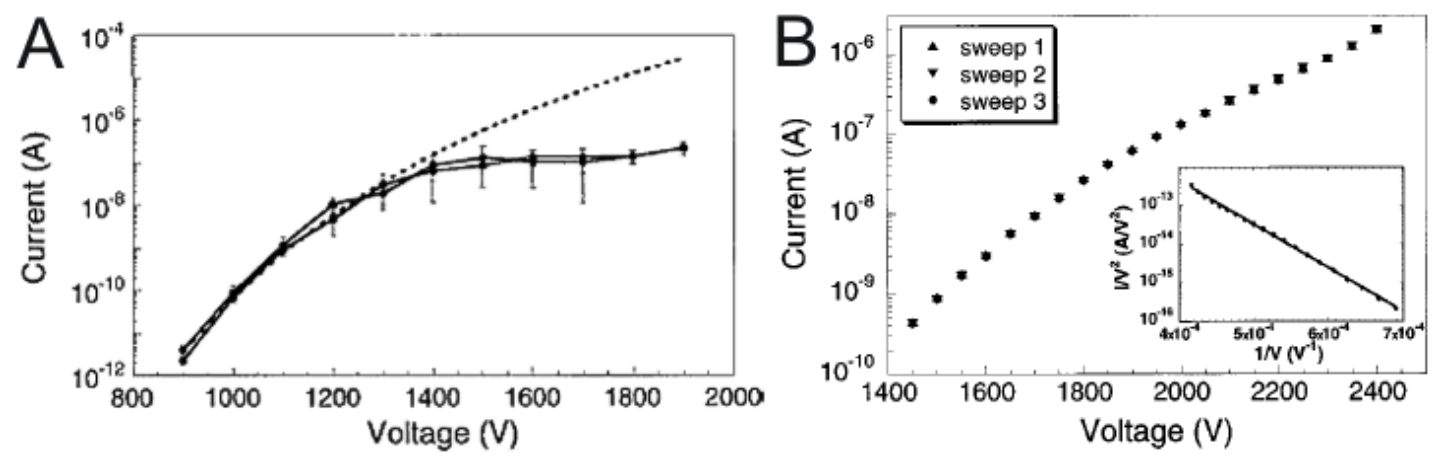

Figure 1-15: Current vs. voltage plots of A) an individual SWNT displaying current saturation behaviour - the dashed line shows the Fowler-Nordheim fit - and B) a clean SWNT showing no current saturation, with the Fowler -Nordheim fit inset. ${ }^{42}$

Conversely, $\mathrm{Xu}$ et al. found non-saturating emission behaviour in individual MWNTs by in situ TEM measurements of the current-voltage relationship. ${ }^{43}$ The authors found that the experimental data fit well to theoretical calculations based on a tip-flat model, where the emission comes from a tip and it has a flat counter electrode. Chai and Chow investigated the field emission from the sidewall of individual MWNT loops, as illustrated in Figure 1-16. ${ }^{44}$ They found a field enhancement factor of 400,000 , which denotes a major increase in the maximum electron emission over the average emission. 


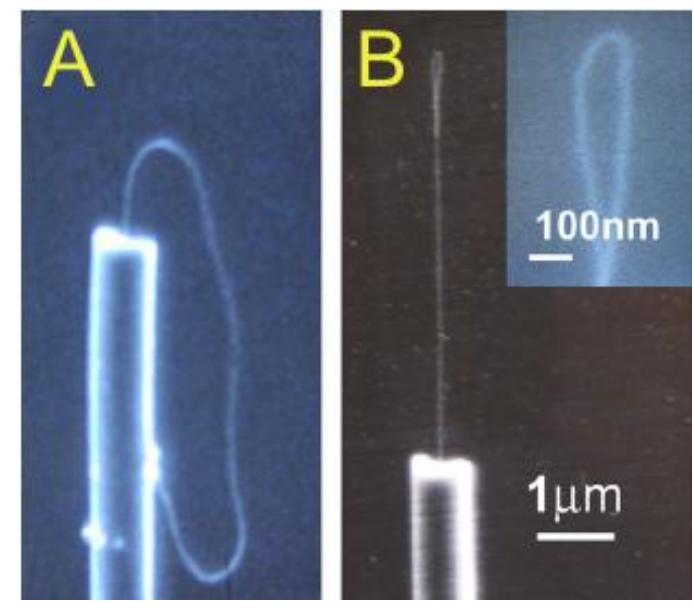

Figure 1-16: SEM image of A) a MWNT loop before high emitting current treatment and B) after treatment. ${ }^{44}$

This diverse range of electronic properties, as well as others not mentioned, makes nanotubes an interesting and appealing target for new, efficient technologies.

\section{Chemical}

A large portion of the intense interdisciplinary research into CNTs now focuses on their chemistry. The reason for this is the immense range of possible future applications as a result of the functionalisation of CNTs.

Generally, as-grown CNTs are very difficult to work with because of their very low solubility in all solvents and high stability under many conditions. Nonetheless, the chemistry of CNTs can be loosely classified into two categories: endohedral filling, and sidewall functionalisation.

The first category, endohedral filling, makes use of the hollow nature of CNTs by filling the inner cavity of the tube with another material or molecule. A wide range of substances can and has been encapsulated within the cavity of CNTs - especially 
considering the diverse range of tube diameters that can be made - including metals ${ }^{45-47}$ and metal compounds, ${ }^{48,49}$ fullerenes ${ }^{50,51}$ and metallofullerenes, ${ }^{52-54}$ and liquids and gases such as water ${ }^{55,56}$ and hydrogen. ${ }^{57}$

However, it is the placement of organic molecules or biomolecules within the tube cavities that is perhaps the most interesting. Davis and associates were able to encapsulate and immobilise the rabbit liver protein $\mathrm{Zn}_{2} \mathrm{Cd}_{5}$-metallothionein and the enzyme $\beta$-lactamase 1 inside SWNTs due to strong interactions with the internal surfaces ${ }^{58}$ while Ito et al. have shown that it is possible to observe the transport of DNA molecules through a MWNT membrane using fluorescence spectroscopy. ${ }^{59}$ These studies pave the way for further work into new forms of drug delivery using targeted drug molecules and species encapsulated within CNTs.

Conversely, sidewall functionalisation - as its name suggests - involves the substitution of different functional groups onto the outside walls and ends of CNTs. Often the purpose of derivatisation is to modify the chemistry of the tube walls in order to increase solubility in solvents, i.e. by increasing CNT hydrophilicity. Again, there are numerous approaches towards functionalising CNTs, as the CNT surface can be considered a surface of $\mathrm{sp}^{2}$ carbons. Therefore, almost any standard chemical functionalisation involving a $\mathrm{C}=\mathrm{C}$ bond can be successfully carried out, including halogenation, ${ }^{60}$ hydrogenation, ${ }^{61}$ cycloaddition, ${ }^{62}$ radical addition, ${ }^{63,64}$ electrophilic addition ${ }^{65}$ and nucleophilic addition. ${ }^{66}$ The downside to these procedures is that the electronic framework of the CNTs is destroyed by substituting across the double bonds. 


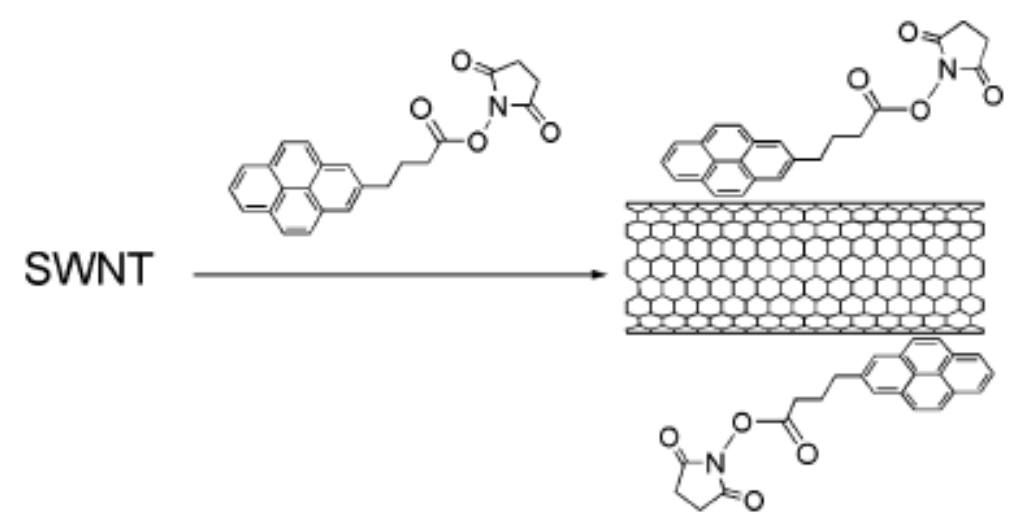

Figure 1-17: Non-covalent $\pi-\pi$ stacking interactions between CNTs and pyrene derivatives. ${ }^{67}$

However, there are other methods of binding molecules to CNT walls using noncovalent interactions such as van der Waals forces and $\pi-\pi$ stacking. These approaches do not directly affect the CNT framework and so would reduce the effect on their electronic properties. In this way, biomolecules can be bound to a CNT surface, as in Figure 1-17 above, as well as forming strong nanotube-polymer composites with a wide range of different polymers, either by individual fibre polymer wrapping or through dispersion within a polymer matrix.

One other major benefit of sidewall functionalisation is that it can enable one to tether CNTs to a desired substrate using a suitable linker. Below, Figure 1-18 depicts one such example of using an aminothiol linker to couple an oxidised nanotube to a gold surface, from work by Liu et al. ${ }^{68}$ This may be advantageous for several reasons, though it is particularly useful for employing CNTs in devices and sensor applications. 


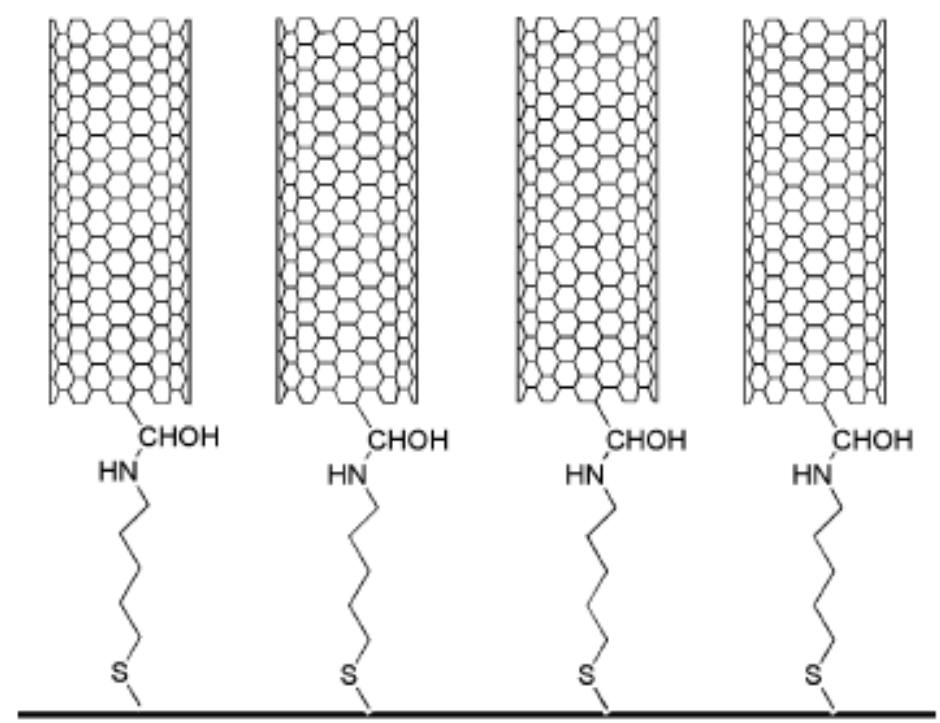

Au surface

Figure 1-18: Controlled tethering of oxidized CNTs onto a gold surface using aminothiol linkers. ${ }^{67}$

As one can see, although CNTs are normally very stable and difficult to work with, there is a wide range of methods to derivatise CNTs, either to improve their workability, for use in devices and sensors, or to attach therapeutic molecules. These remarkable chemical properties, as well as the physical and electronic properties, make nanotubes a highly attractive option for further study in many applications, particularly in sensors and drug delivery.

\subsection{Synthetic techniques}

The growth of carbon nanotubes can be achieved using a host of methods, ranging from electrochemical or wet chemical techniques to solar heating. Many of these approaches involve different procedures for vaporising a carbon source in the presence of a suitable growth catalyst, typically a transition metal. Here, the four most common techniques for carbon nanotube growth will be discussed: arc 
discharge, laser ablation, high pressure disproportionation of carbon monoxide and chemical vapour deposition (CVD).

1. Arc discharge (also known as electric arc or carbon arc)

The arc discharge method was the first popular method for forming carbon nanotubes. It was the technique used to produce Iijima's first historic samples, ${ }^{1}$ but also was used by Abrahamson much earlier. ${ }^{6}$ Depicted in Figure 1-19, the technique is usually performed at low pressures under inert atmosphere, and involves the formation of a carbon vapour by inducing a current arc between two graphite electrodes. As the large current flows ( $\geq 100 \mathrm{~A}$ ), it increases the temperature at the anode $\left(\mathrm{ca} .3000{ }^{\circ} \mathrm{C}\right)$ such that the carbon at the anode sublimes. From here it is transported to the cathode where it is deposited in the form of MWNTs. SWNT growth can be encouraged by co-evaporating metal atoms - usually iron or cobalt with the carbon source. ${ }^{9,10}$

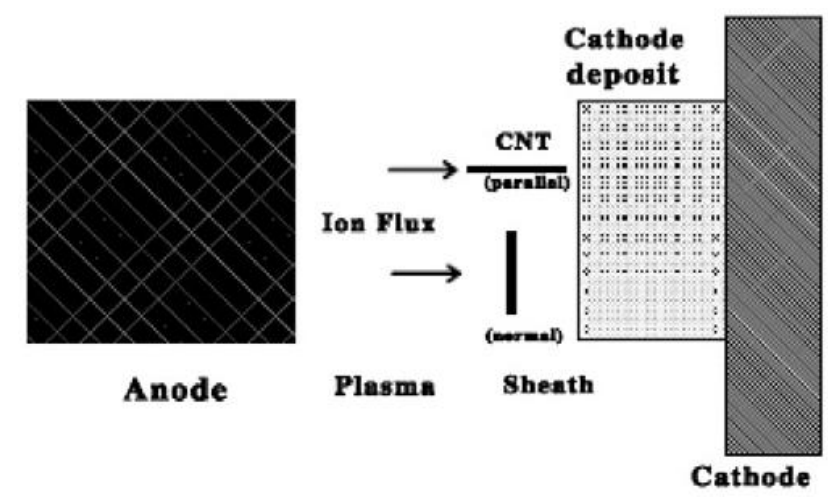

Figure 1-19: Schematic for the apparatus and operation of the arc discharge method. ${ }^{69}$

This method has proved popular for the growth of CNTs and is currently the most commonly used method for commercial production. This is due to the creation of 
high purity products with highly perfect structures, coupled with the capability for production on a reasonably large scale, first shown by Ajayan and Ebbesen. ${ }^{70}$

On the other hand, there are some disadvantages of using an arc method. Firstly, this method is only capable of batch manufacture - where the products are formed and later collected once production has finished - with no possibility of continuous fabrication. Secondly, due to the very high temperatures involved in the process, it is not possible to grow the nanotube products directly onto a substrate. This means that if they are to be used in the electronics industry for instance, the tubes must first be collected and subsequently attached to a substrate.

\section{Laser ablation (or pulsed laser vaporisation)}

Laser ablation was first used to produce carbon nanotubes by Smalley in $1995 .^{71}$ Shown in Figure 1-20, the process is similar to that of arc discharge, except here the carbon vapour is produced by irradiating a graphite target with a high-power laser. Again, the method is undertaken in a low pressure, inert atmosphere at high temperature $\left(1200{ }^{\circ} \mathrm{C}\right)$ and the carbon products are subsequently deposited on the vessel walls or on some cooled surface.

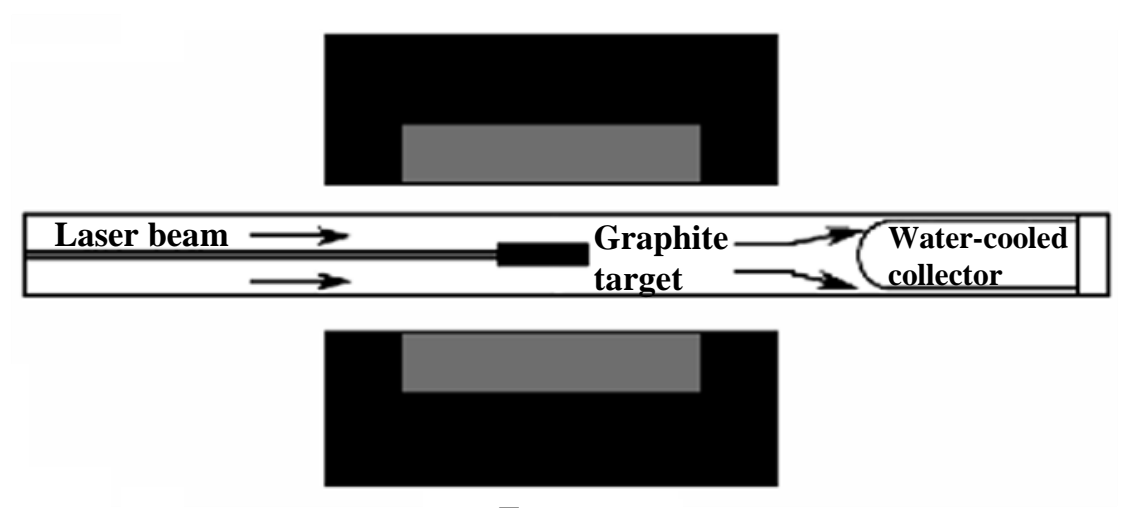

Furnace 
The advantages of this process are the very high yields $(\sim 70 \%)$, high purity products and the ability to tailor nanotube diameter with careful temperature control. However, this technique is also the most expensive of the commonly used methods.

3. High pressure disproportionation of carbon monoxide (HiPCO)

The HiPCO process for the growth of CNTs was first used in 1996 by Smalley's group at Rice University. ${ }^{72}$ The procedure employs the Boudouard reaction, the disproportionation of carbon monoxide at high pressure to form carbon with carbon dioxide as a by-product, shown in Equation 1-3:

$$
2 \mathrm{CO} \stackrel{5-10 \mathrm{~atm}}{\longrightarrow} \mathrm{C}+\mathrm{CO}_{2}
$$

Similar to other synthetic techniques, a metal catalyst such as molybdenum, iron or cobalt is used to promote the favoured growth of CNTs over amorphous carbon. Depicted in Figure 1-21, the process operates by injection of a cold stream of carbon monoxide and catalyst - typically pentacarbonyl iron - into a high pressure system of hot carbon monoxide, where CNTs begin to grow above $900{ }^{\circ} \mathrm{C}$.

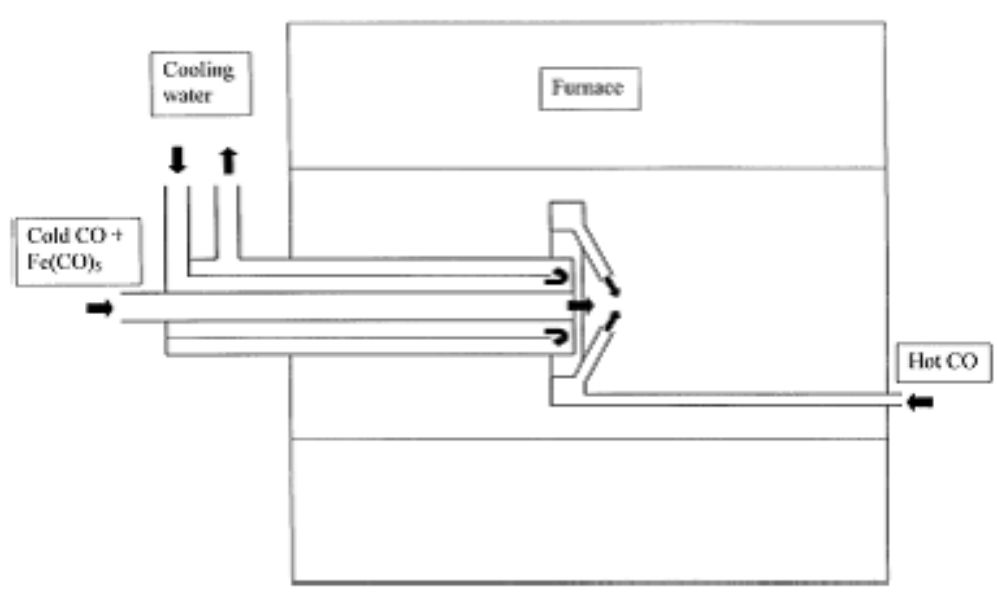

Figure 1-21: Schematic of the reactor used by Smalley in the HiPCO process. ${ }^{73}$ 
The advantages of this process are that the CNT products are generally very pure and selective, with some groups claiming a 90\% weight of SWNTs with average diameters less than $1 \mathrm{~nm} .^{74}$ Additionally, the CNTs are produced in a gas stream that can be directed onto any desired substrate and will continue to grow as long as there is a supply of carbon monoxide and catalyst, so this procedure can be operated continuously.

However, the obvious disadvantages to this method are the high temperatures and pressures necessary to promote CNT growth. For efficient CNT growth, temperatures above $900{ }^{\circ} \mathrm{C}$ and pressures $\sim 10-15$ atm must be attained. At pressures and temperatures below this the formation of amorphous or graphitic carbon is preferred. ${ }^{74}$

\section{Chemical vapour deposition (CVD)}

CVD is a highly versatile approach for producing nanotubes and is perhaps the most commonly published technique for nanotube growth. Depicted in Figure 1-22, the process involves decomposing a carbonaceous precursor at high temperature under an oxygen-free, reducing atmosphere to produce nanotubes. The carbonaceous precursor may be a carbon gas, methane or ethylene for instance, or in the form of a volatile hydrocarbon solvent such as ethanol, and is generally fed into the reactor with some inert carrier gas. In the reaction chamber, the precursor is decomposed in the presence of a catalyst - again, usually a transition metal such as iron, cobalt or some similar combination of metals. The catalyst can be introduced in a number of different ways. Often, a substrate is coated with a thin film of catalyst by sputtering or some other casting method. Alternatively, a volatile organometallic precursor 
such as ferrocene may be either evaporated or, when using a hydrocarbon solvent precursor, it may be dissolved in solvent. The catalyst is then converted into the active catalyst by in situ reduction, or decomposition in the case of an organometallic precursor, to form nanosized catalyst particles. A reducing gas, hydrogen or ammonia, is often added to aid in the reduction of the catalyst and to decrease particle size. ${ }^{75-77}$

Decomposition of the carbon precursor can also be achieved in many ways, though the two most common methods are simple thermal decomposition or by forming a plasma, as in plasma enhanced CVD (PECVD). Several techniques have been employed to produce a plasma, including radio frequencies (RF-), direct current (DC-) and microwaves (MW-). Nanotube growth is achieved at high temperatures, typically $700-1000{ }^{\circ} \mathrm{C}$ depending on the catalyst and carbon precursor employed, although the use of a plasma can reduce this to $450-700{ }^{\circ} \mathrm{C}$.

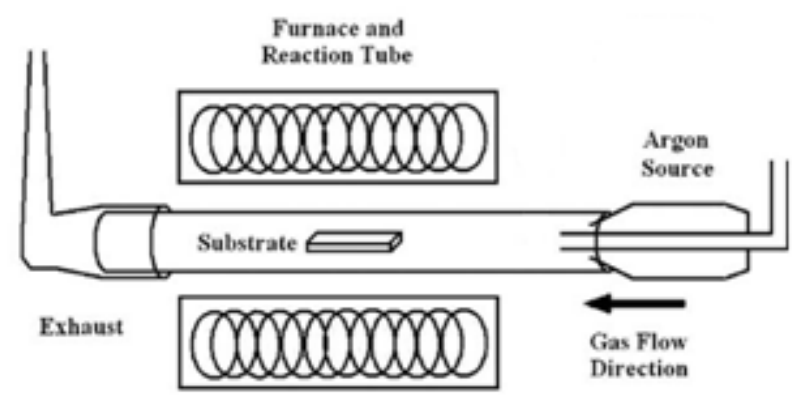

Figure 1-22: Simple schematic of a typical CVD set-up. ${ }^{78}$

CVD is often considered to be the most promising technique for the bulk synthesis of CNTs with tailored structures and diameters for several reasons. Firstly, it is a relatively cheap process and reasonably simple to scale up, even showing promise for the continuous production of nanotubes. ${ }^{79}$ Also, because of the lower 
temperatures relative to arc discharge and laser ablation, it is possible to grow CNTs directly onto a substrate and by careful catalyst pre-treatment and patterning, it is possible to produce aligned nanotubes in selected regions. ${ }^{77,80}$ This is particularly beneficial when making nanotube devices for use in electronics, where good contact between tube and substrate is necessary, and where device design is important. Finally, because of the large number of parameters crucial to the CVD conditions carbon precursor, catalyst, carrier gas, reducing gas, temperature etc. - it offers the ability to tune the nanotube product by careful control of the conditions. Conversely, this also means there are a lot of parameters to manage when employing this method.

The main disadvantage to the CVD method is that the carbon products tend to be less highly graphitised. The increased incidence of defects means the CNTs exhibit inferior properties, particularly decreased mechanical strengths.

\subsection{Growth mechanisms}

One of the important aspects in the investigation of CNTs is the ability to control the nanotube growth in terms of helicity, diameter, length and number of walls. In order for this to be possible, a good appreciation of the mechanism of formation is crucial. Presently however, the mechanism is not well understood although there are several reasons to explain this lack of knowledge.

Firstly, there are a number of synthetic techniques available for CNT growth, so it is difficult to reconcile the evidence from different methodologies in order to uncover an all-encompassing mechanism. Furthermore, there are many parameters to 
describe the growth conditions of CNTs - including reaction temperature, catalyst and carbon precursor - each having some effect on the growth conditions and hence on the growth mechanism. Thus, with so many different parameters to investigate for a single synthetic method, it is often difficult to unravel the individual effects to determine an underlying mechanism.

Finally, before now there were heavy limitations on the ways that the mechanisms could be investigated. Previously, most analysis was performed ex situ, which involved carrying out the synthetic procedure for some time, removing the sample and then performing analysis. This meant that one had to infer the method of growth based on the final product. It has only been more recently that in situ techniques, such as environmental SEM and TEM, have been developed to analyse carbon nanotube growth in real-time. ${ }^{81-88}$

In spite of these problems, some have attempted to explain carbon nanotube formation from both empirical and calculated data. Many of these explanations use some form of the vapour-liquid-solid (VLS) mechanism, first proposed by Wagner and colleagues to account for the formation of silicon whiskers. ${ }^{89}$ This idea was later adapted to describe CNT formation by Saito et al. ${ }^{90}$ In the VLS mechanism, growth is achieved through the interaction of three separate phases. First, at high temperature a liquid catalyst particle is formed. This can be achieved either by depositing a nanoparticle film or by in situ formation, through condensation of a metal or organometallic vapour, or reduction of a pre-catalyst film. A carbonaceous vapour is then introduced and adsorbed onto the surface of the catalyst particle, whereupon it decomposes at high temperature to form smaller carbon fragments that 
dissolve into the liquid metal phase, until such time as the catalyst particle becomes supersaturated. At this point, the carbon begins to precipitate and is extruded from the particle to form CNTs.

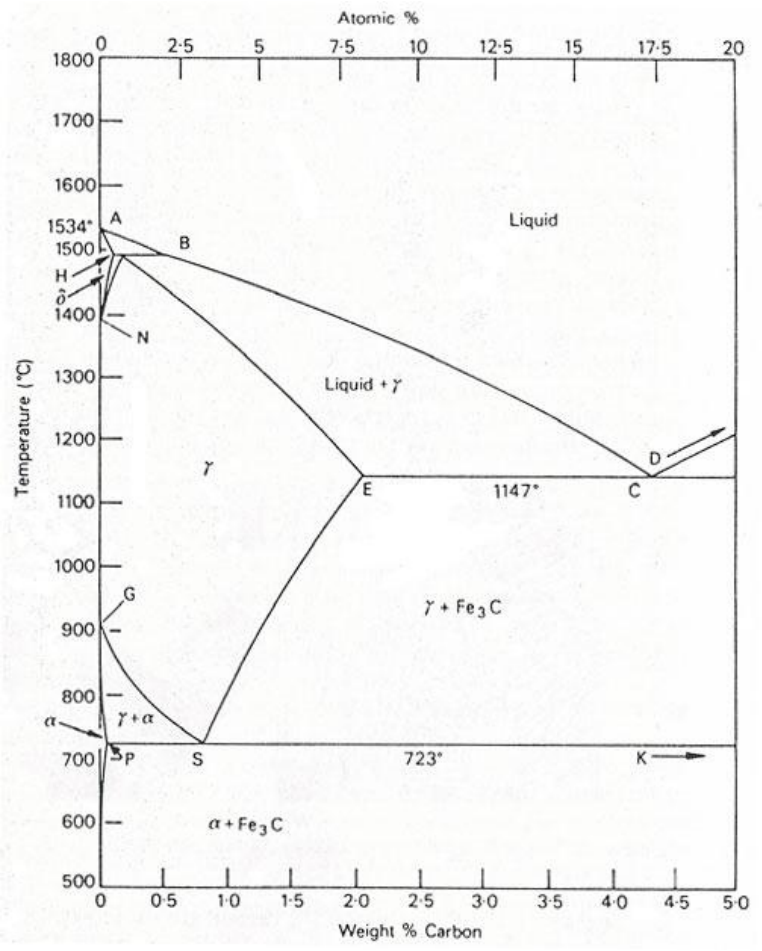

Figure 1-23: Binary phase diagram for the Fe-C system. ${ }^{91}$

There is plenty of experimental evidence within the literature to support the VLS mechanism. While the melting point of iron in the bulk Fe-C binary phase system is much higher than in normal synthetic conditions $-1175{ }^{\circ} \mathrm{C}$ from Figure 1-23 Harutyunyan et al. were able to show that the eutectic point between the solid and liquid phase of the Fe-C binary phase diagram was strongly dependent on the size of the iron catalyst particle. ${ }^{92}$ As illustrated in Figure 1-24, as the size of the catalyst particle decreased, so too did the temperature at which it melted, therefore it was possible for small catalyst particles to melt at much lower temperatures than expected. Meanwhile, Liu and Fan were able to show that the deposited carbon 
must be extruded from the catalyst particle, as opposed to being deposited directly onto the tube, by employing ${ }^{12} \mathrm{C}_{2} \mathrm{H}_{4}$ followed by ${ }^{13} \mathrm{C}_{2} \mathrm{H}_{4}$ as the carbon source during the course of their CVD synthesis. ${ }^{93}$ Subsequent Raman analysis showed that the ${ }^{13} \mathrm{C}$-labelled atoms were found in regions of the tube closer to the attached catalyst particle, as would be expected if the carbon fragments must dissolve in the catalyst particles. Other evidence offered by Kim and co-workers showed that using different metal catalysts resulted in different CNT growth rates, suggesting that carbon diffusion was a rate limiting step. ${ }^{94}$

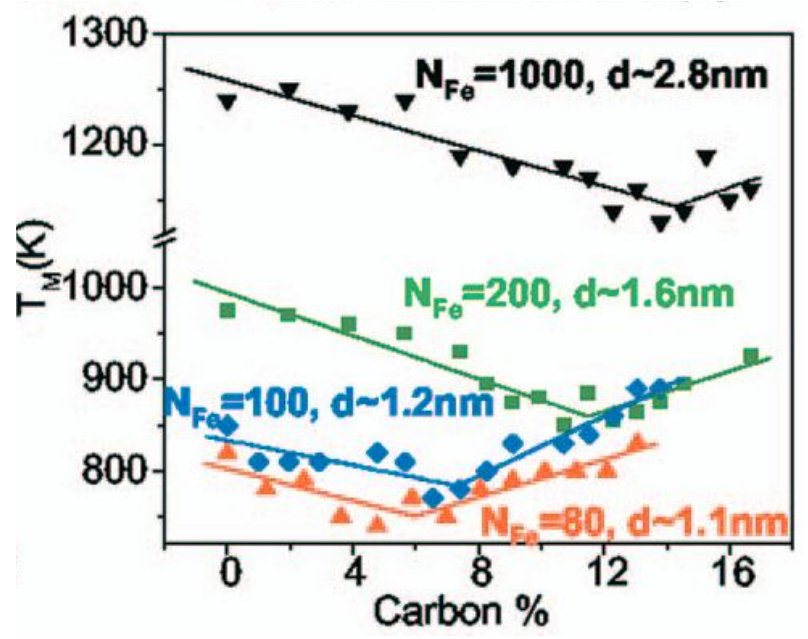

Figure 1-24: Binary phase diagram of the Fe-C system, focussing on the effect of catalyst particle size on the phase boundary between the solid and liquid phases and the associated eutectic point. ${ }^{92}$

To describe the driving force for carbon dissolution within the liquid metal particle and the subsequent precipitation of carbon nanofibres (CNFs), Baker and co-workers suggested that a temperature gradient across the catalyst particle was needed. ${ }^{3}$ The authors proposed that fibre growth was a product of four separate steps: decomposition of the hydrocarbon precursor followed by rapid carbon diffusion into the catalyst particle to form metastable metal carbides, precipitation onto the back 
face of the particle to form the fibre body and finally the formation of a carbon coat on the surface of the particle leading to termination of fibre growth. The authors suggested the carbon dissolution process is an exothermic reaction, thus raising the temperature of the particle on the front surface. Conversely, the precipitation reaction is an endothermic process, causing a lower temperature at the opposite face of the particle. This temperature gradient was thought to be the driving force through which the continued growth was promoted (Figure 1-25A). This suggested bulk diffusion was the limiting factor in the growth of CNFs. Ding et al. later adapted this idea of bulk diffusion driven by a temperature gradient to explain the growth of SWNTs. ${ }^{95}$

In contrast, Sinnott and colleagues propose a concentration gradient driven process where the precipitation of the $\mathrm{CNT}$ at the back face of the particle causes a low local carbon concentration inside the particle surface. ${ }^{96}$ Where the hydrocarbon decomposition occurs at the particle surface there is a high carbon concentration and the concentration difference between these two regions causes the transport of carbon from the high concentration region to the low region, where it is precipitated (Figure 1-25B). 


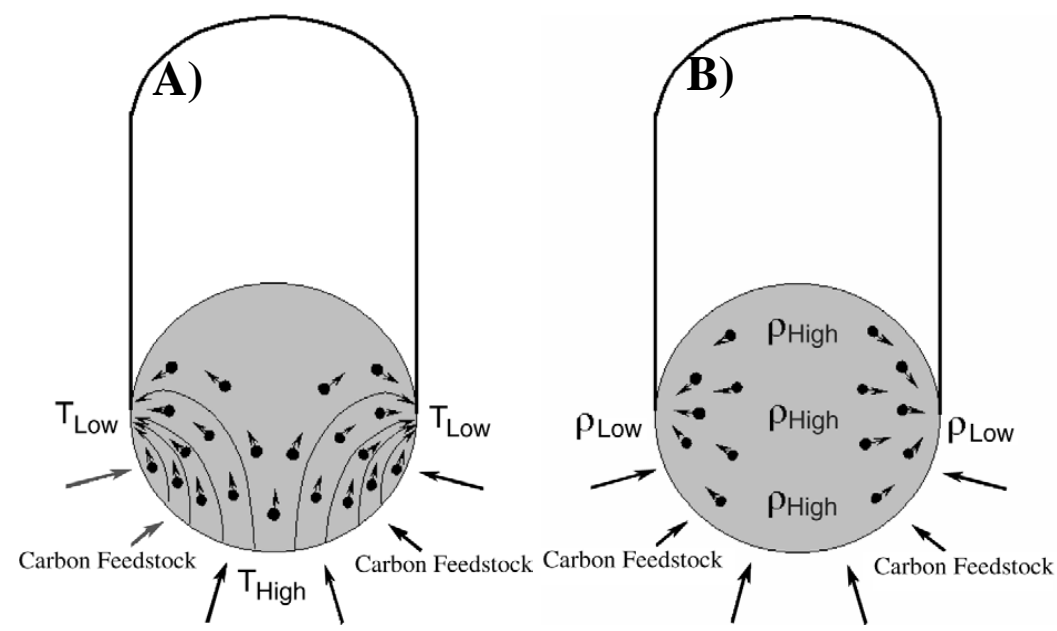

Figure 1-25: Comparison of the models for the carbon dissolution and precipitation in a metal catalyst particle driven by A) a temperature gradient across the particle and B) a concentration gradient across the particle. ${ }^{95}$

On the other hand, in their early nanotube work Oberlin et al. deduced a mechanism whereby bulk diffusion is irrelevant, but instead the surface diffusion of carbon along the particle surface is the important factor, as shown in Figure 1-26. ${ }^{4}$ This was later (and apparently independent of the earlier report) proposed by Hofmann and co-workers and also Helveg et al., to describe the growth of CNFs. ${ }^{97,98}$

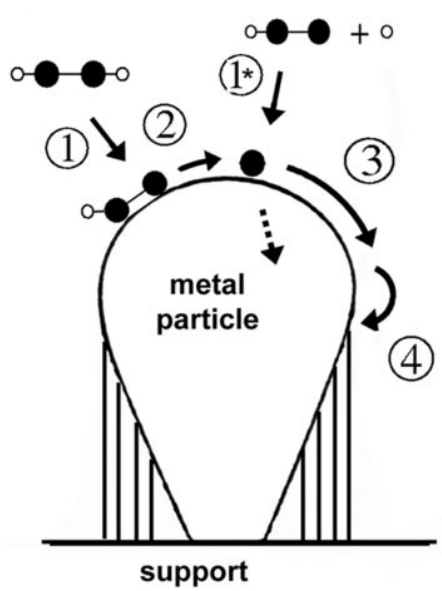

Figure 1-26: Model depicting the decomposition of acetylene and subsequent diffusion of carbon species along the surface of a catalyst particle in the growth of CNTs. ${ }^{99}$ 
In fact, Helveg and colleagues' report of the in situ imaging of CNF growth, ${ }^{97}$ in conjunction with newer evidence based on the low temperature growth of SWNTs, ${ }^{75}$ casts doubt on whether the VLS mechanism is necessary for nanotube growth. The authors grew CNFs from decomposition of methane over supported nickel nanoclusters and by following the reaction with in situ TEM, the authors were able to demonstrate the presence of lattice fringes in the $\mathrm{Ni}$ catalyst clusters. This suggests that rather than being molten, in fact, the nickel catalyst clusters remained crystalline throughout, implying the growth proceeds through some vapour-solid mechanism. In addition, Cantoro and co-workers demonstrated the growth of dense fields of SWNTs from the thermal decomposition of acetylene at temperatures as low as $350{ }^{\circ} \mathrm{C}^{75}$ The authors employed a cold wall, catalytic CVD method, where a $\mathrm{Si} / \mathrm{SiO}_{2}$ substrate with an evaporated thin catalyst film was heated using a specially designed, resistively heated, graphite stage. The substrate and catalyst film were heated under ammonia or hydrogen to form discrete catalyst islands, the gas evacuated and undiluted acetylene introduced. At these low temperatures it is virtually inconceivable that the catalyst particles are a liquid. Instead, the authors suggest that chemisorbed species such as hydrogen atoms can loosen the particle surface, aiding the diffusivity of carbon along the surface.

Whatever the debate, these ideas lead to two complementary theories for the mechanism of CNT growth; base or root growth and tip or cap growth, depicted in Figure $1-27 .^{3}$ In base growth, the catalyst particle is located at the substrate surface. The process is thought to follow the VLS mechanism where the carbon vapour dissolves into the catalyst particle and when the dissolved carbon subsequently 
precipitates, the tube grows up from the surface, leaving the catalyst particle attached to the substrate surface. The consequence of this is that for large aligned fields of CNTs, as the growth proceeds the carbon vapour must somehow diffuse through the already grown forest in order to access the catalyst particle. Alternatively, in the tip growth mechanism, as the dissolved carbon precipitates to form the tube, the tube forces the catalyst particle away from the substrate surface resulting in a metal nanoparticle enclosed within the tube walls. The deciding factor between these two methodologies is thought to be the relative adhesion the metal particle has for the substrate surface. ${ }^{100}$ Low surface adhesion favours the tip growth mechanism, while high surface adhesion favours the base growth process.

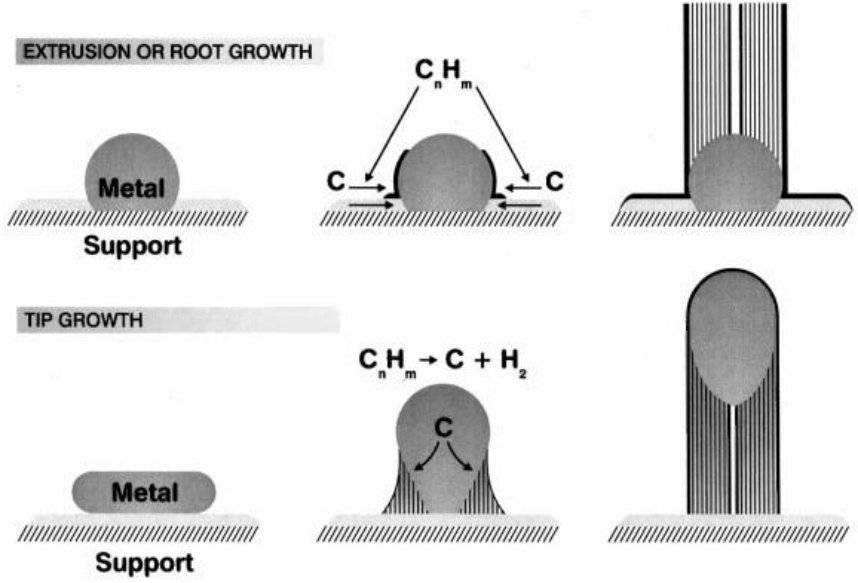

Figure 1-27: Model of the base and tip growth mechanisms. ${ }^{78}$

The chief alternative to the VLS mechanism was suggested by Thess et al., and is known as the 'scooter mechanism'. ${ }^{101}$ Depicted in Figure 1-28, this idea was proposed in order to explain the formation of perfect hemispherical caps with the absence of any encapsulated catalyst particles in SWNTs made using a laser ablation method. As proposed, rather than a tube growing up from a catalyst seed particle as in the VLS mechanism, initially a single metal atom 'scoots' around the open edge 
of the tube during growth, helping to anneal any defects within the tube structure. As the growth continues, more metal atoms will diffuse to the open tube edge and condense to form a small metal cluster. The metal cluster helps to catalyse the decomposition of larger carbonaceous deposits, which then diffuse through the metal and extend the growth of the tube at the edge. The metal cluster is later expelled either by evaporation or by substitution as the tube closes.

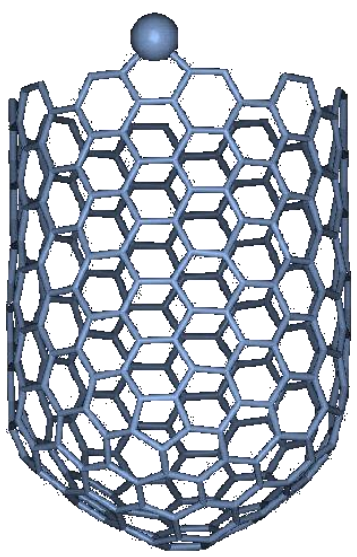

Figure 1-28: Model of the 'scooter' mechanism. ${ }^{101}$

As one can see, there are many different theories to explain nanotube growth, though none have been universally accepted. The reason for this is the diverse range of factors that determine the growth often make generalisations on the formation mechanism difficult. Nevertheless, new in situ analytical techniques are now helping to give researchers a better understanding of the growth processes to aid nanotube growth control in the future.

\subsection{Characterisation}


In order to determine the effect of different CVD reaction conditions and, more specifically, of different substrates, CNT products are characterised using a variety of techniques, ranging from imaging to thermal analysis.

The key methods for determining nanotube morphology and composition involve direct imaging using electron microscopy. Electron microscopy is similar to regular optical microscopy, where a beam of light is focussed onto a sample and then a lens or series of lenses, generally made from glass, is employed to focus the scattered or refracted light to form an image. Optical microscopy performed with a compound microscope that employs a condenser lens to focus the incident beam, an objective lens to refocus the scattered beam into an image and then generally a third lens to enlarge the image, can achieve maximum resolutions of approximately $0.2 \mu \mathrm{m}$ or 1500x magnification. As such, regular optics is incapable of resolving much smaller objects, such as those on the nanometre scale. Instead, electron microscopy is employed where, rather than focussing a beam of light onto a sample, a beam of high energy electrons is used. This of course means that the typical refractive lenses used in optical microscopy are ineffectual and are replaced by arrays of electromagnets, which focus the electron beam by generating and precisely manipulating a magnetic field. Obviously, the resulting image is a beam of electrons and so cannot be directly viewed by the naked eye. Instead, the beam is projected onto a suitable detector such as a scintillator detector, phosphor screen, photographic film, or charge-coupled device.

Transmission electron microscopy (TEM) is one form of electron microscopy that is used to investigate carbon nanotubes. TEM is highly analogous to compound 
optical microscopy where in TEM a beam of high energy electrons (generally hundreds of $\mathrm{keV}$ ) is directed through a thin sample film and a cross sectional image of the transmitted electrons is produced on the other side. As the beam passes through the sample, some electrons are scattered and reflected by atoms within the sample, and so will not carry on to strike the screen. The image formed is a contrast representation of light and dark areas, as heavy nuclei scatter the beam more intensely than lighter nuclei due to their higher density. The major advantage of TEM is that the beam passes through the sample to generate a cross-sectional view, thus providing information on internal structure. The high voltage beam also means the image resolution can be very high, typically about $0.2 \mathrm{~nm}$ at magnifications of about 2,000,000x. However, a large, million-volt transmission electron microscope can resolve separations less than $0.05 \mathrm{~nm}$ and achieve magnifications of up to $50,000,000 x$, enough to discern individual atoms.

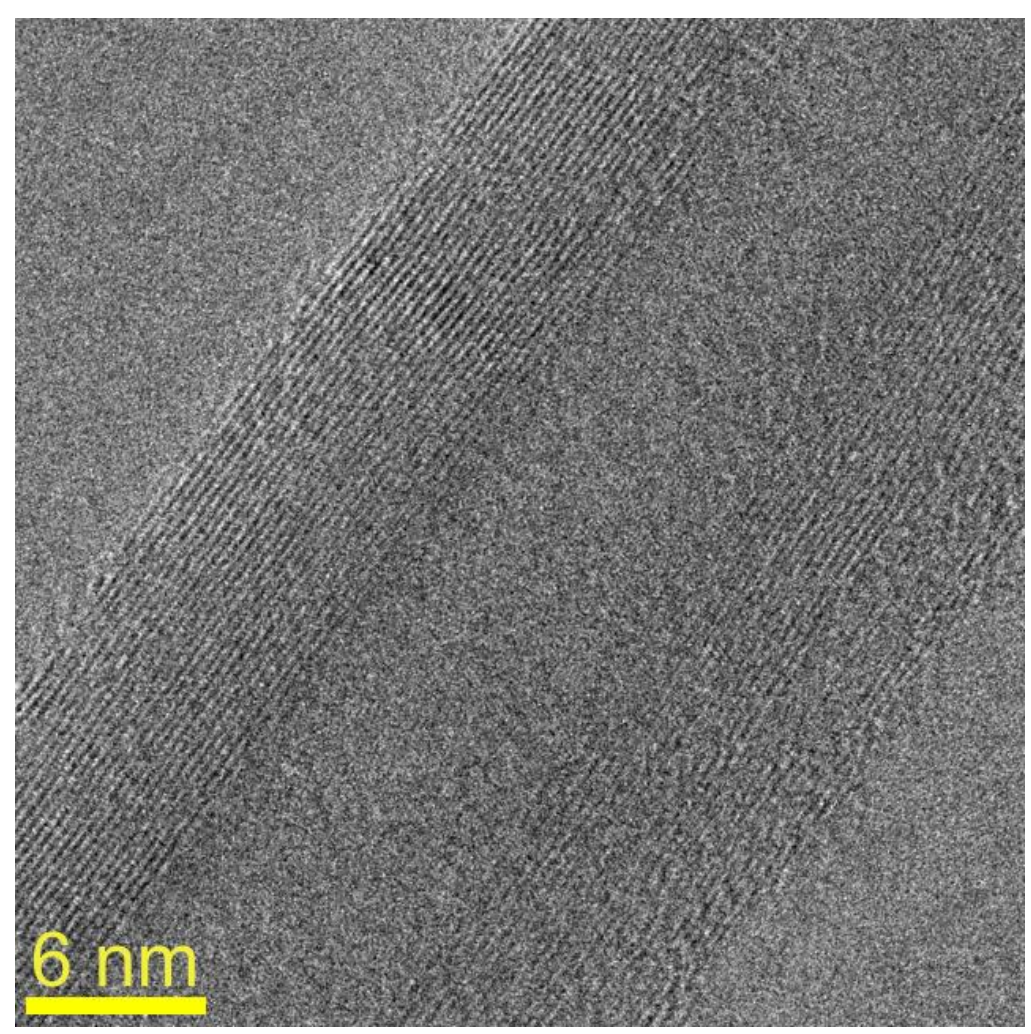


Furthermore, when an electron from the primary beam collides with an electron from an inner orbit of an atom within the sample, the incident electron is scattered and the core electron is emitted from the atom, resulting in an electron hole. An electron from a higher energy orbit can then drop from this higher orbit into the newly vacated hole, releasing the excess energy in the form of an X-ray. The emitted X-rays have discrete energies equal to the drop in energy of the electron and the distribution of these energies is characteristic of particular atoms. Hence, the emitted X-rays can be detected and analysed using energy dispersive X-ray spectroscopy (EDS or EDX) to determine the elemental composition of the sample. One can go further and build a map of elemental distribution by coupling EDS with scanning transmission electron microscopy (STEM), where the sample is raster scanned over an area. These techniques, among others, mean that TEM is a powerful instrument for investigating the internal atomic structure of a specimen. For carbon nanotubes in particular, TEM is useful for accurately determining the diameters of individual tubes and the number of constituent walls (Figure 1-29). The technique is also effective for detecting the presence of trapped catalyst particles.

Although TEM can achieve high magnifications and resolutions, there are some drawbacks. Firstly, because the electrons pass through the sample to produce a cross-sectional image, it is almost impossible to interpret height within the sample. As such, TEM images appear two-dimensional. This can lead to structural confusion caused by tube stacking and make it difficult to conclusively deduce the true shape of an item in a particular sample from TEM alone. Secondly, due to the 
very high magnifications, TEM only has a limited field of view. This means it is difficult to look at large bundles or arrays of tubes and makes it possible for small impurities to appear more significant than they truly are. Therefore, in order to properly examine nanotube morphology and the structure of large fields, it is necessary to complement TEM with other electron microscopy techniques.

Scanning electron microscopy (SEM) is one other form of electron microscopy. Scanning electron microscopes can achieve resolutions of $\sim 1 \mathrm{~nm}$ and typical magnifications of $650,000 x$, although better instruments can achieve up to 2,000,000x. With SEM, a focussed beam of electrons is raster scanned across a large sample area. As the high energy electron beam interacts with the sample surface, incident electrons collide with electrons inside the sample, with some transferring a little of their energy - known as inelastic scattering - and ejecting these secondary electrons from their orbits. The ejected electrons can then strike a scintillator detector placed near the specimen where it is converted into a digital, grey-scale image, as in secondary electron imaging (SEI). When the secondary electrons shift from their orbits, they collide and exchange energy with many other surrounding electrons and hence the electrons emitted from the sample surface are of low energy $(<50 \mathrm{eV})$ and have a mean free path length of only $1-3 \mathrm{~nm}$ through the sample. As such, secondary electrons originate from near the surface and the detection of these emitted electrons is greatly dependent on the surface topology of the specimen. This gives SEI a high depth of field and a three-dimensional appearance (Figure 1-30A). This, and the ability to scan over a relatively large sample area with high resolution, makes SEI an excellent technique for studying the surface morphology of a sample. 
Again, as for TEM, when the electron beam interacts with the specimen, characteristic X-rays are emitted and can be detected, meaning elemental composition can be analysed using EDS and elemental mapping (Figure 1-30B \& C). Another useful SEM technique for investigating sample composition is Rutherford backscatter (RBS). Here, when the incident beam strikes the sample, a small number of electrons are elastically scattered - collision with no accompanying energy transfer - and these high energy, reflected electrons can be detected to form a contrast image. Due to their low atomic number and electron densities, lightweight elements have a much lower rate of collision, and hence cause fewer backscattered electrons than heavier elements. This not only means heavy elements appear brighter in the ensuing image while less heavy elements appear darker, but the incident beam can penetrate further through lighter elements than heavy elements. This means that one can detect the presence of heavy elements even when trapped within lighter elements. This is particularly useful in CNT research for detecting the presence of transition metal catalyst particles trapped within tube walls. 

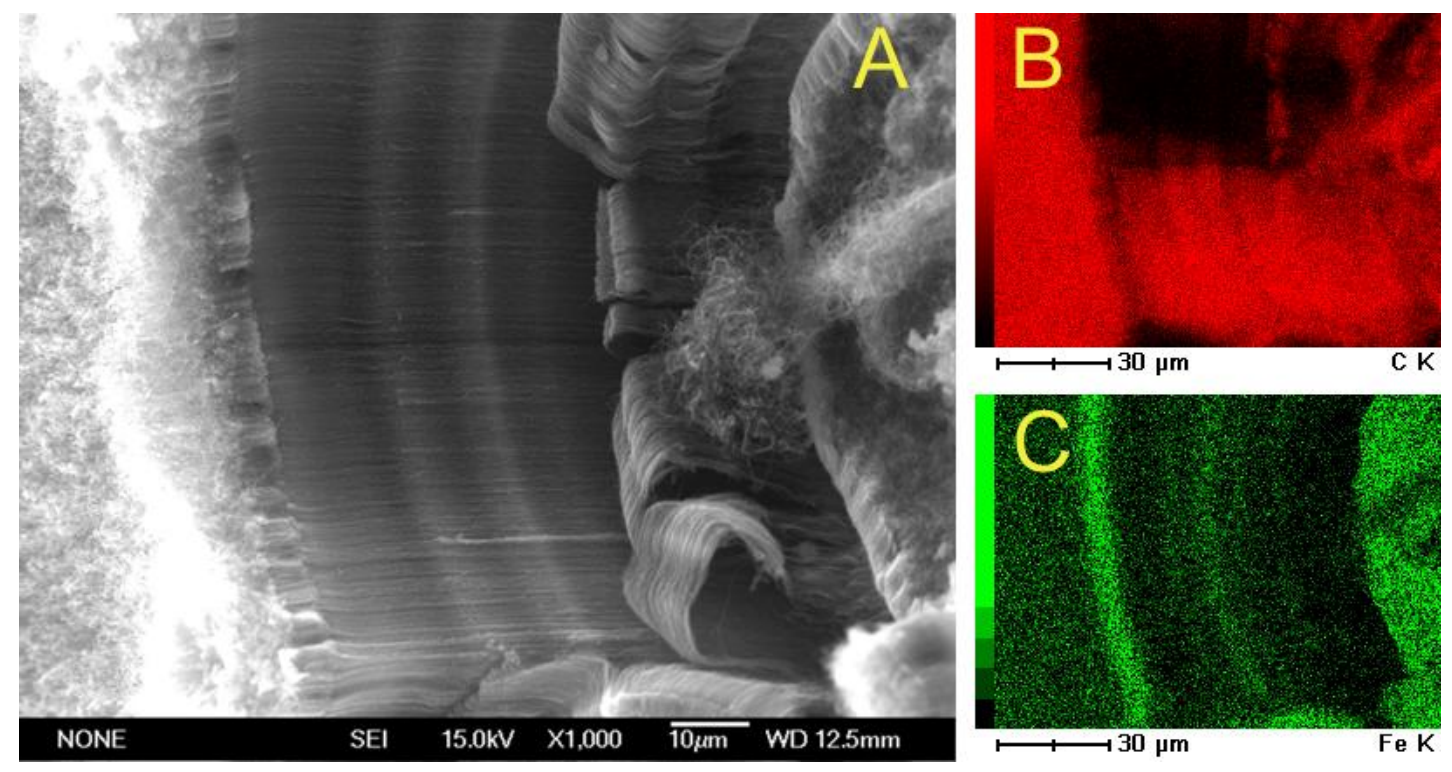

Figure 1-30: Typical SEM image exhibiting large aligned fields of as-grown CNTs. A) is the image taken in SEI mode, while elemental maps of the same area, show the distribution of B) carbon and C) iron.

These three techniques, among others not discussed here, make SEM a powerful analytical tool for investigating new materials. In this research, SEM is particularly useful for analysing the large scale alignment and morphology of carbon nanotube fields, as well as examining their elemental composition and distribution. It is also possible to detect the position of trapped catalyst particles. For these reasons, SEM is an important method of investigation.

To complement electron microscopy and to examine other features of the prepared nanotubes, other analytical techniques are used. To investigate carbon nanotube structure and crystallinity, there are several techniques which could be employed, such as X-ray ${ }^{102}$ and electron ${ }^{9,102}$ diffraction. However, perhaps the most commonly performed and most convenient method is Raman spectroscopy. Raman spectroscopy is a form of vibrational spectroscopy, often complementary to infrared 
(IR) spectroscopy, and was first employed for CNTs by Hiura and associates. ${ }^{103}$ When photons from a laser interact with molecules, the molecules absorb the photon energy and are excited to a virtual electronic state. The molecules will then relax to a ground state and release the excess energy by emitting a photon. Most of the emitted photons will have the same energy as the incident photons, a process known as elastic or Rayleigh scattering. However, a small proportion of the emitted photons will have energies different from the incident photons due to vibrational transitions within the molecule. This phenomenon is known as inelastic scattering and is the basis of the Raman effect (Figure 1-31).

\section{Energy}

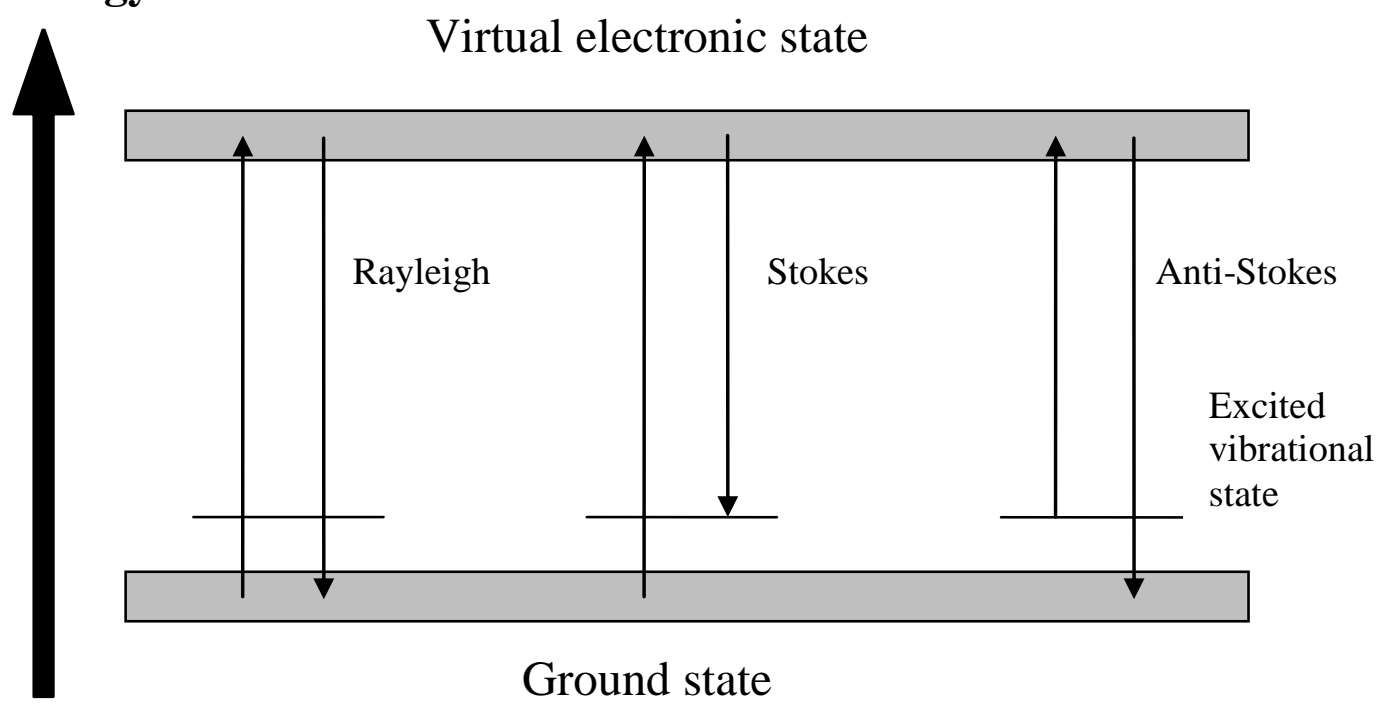

Figure 1-31: Sketch depicting the different forms of scattering when photons interact with molecules.

Stokes scattering occurs when the excited molecule does not drop to the ground state, but instead drops to an excited vibrational state. Hence, there is an observable red shift between the incident and scattered photons. When the scattered photon has a higher energy than the absorbed energy, this is known as anti-Stokes scattering. Anti-Stokes scattering occurs when a vibrationally excited molecule scatters a 
photon, and subsequently drops down to a lower vibrational state, thereby causing a blue shift in the emitted photon. Because the principal state of a molecule at room temperature is the ground state, anti-Stokes scattering is far less abundant than Stokes scattering and so the Stokes spectrum is generally more useful for analysis.

What makes Raman spectroscopy different from IR spectroscopy is the selection rule that governs which vibrational transitions are observable. In IR spectroscopy, only vibrations that result in a change in the dipole moment of a molecule will be observed. However for Raman spectroscopy, only those transitions that change the polarisability of the molecule - the ease with which the electron cloud can be distorted - will be observed. This means that for molecules with a centre of symmetry, the modes which are not IR active are generally Raman active, and vice versa.

The key aspect for carbon nanotube research is that many of the phonon modes of the C-C bond are Raman active, and so we can investigate nanotube structure by examining the stretching frequencies in a Raman spectrum. While the specific phonon interactions are very complex and there is not complete agreement over the influence of parameters such as chirality and tube diameter on the vibrational modes, some generalisations can be made for the features of a Raman spectrum of CNTs. There are numerous stretching modes that can be measured in the Raman spectrum of CNTs, though most of these have a relatively low intensity. Nonetheless, there are three key features with high intensities that are of interest when examining CNTs: radial breathing modes, the $\mathrm{G}$ band and the $\mathrm{D}$ band. 

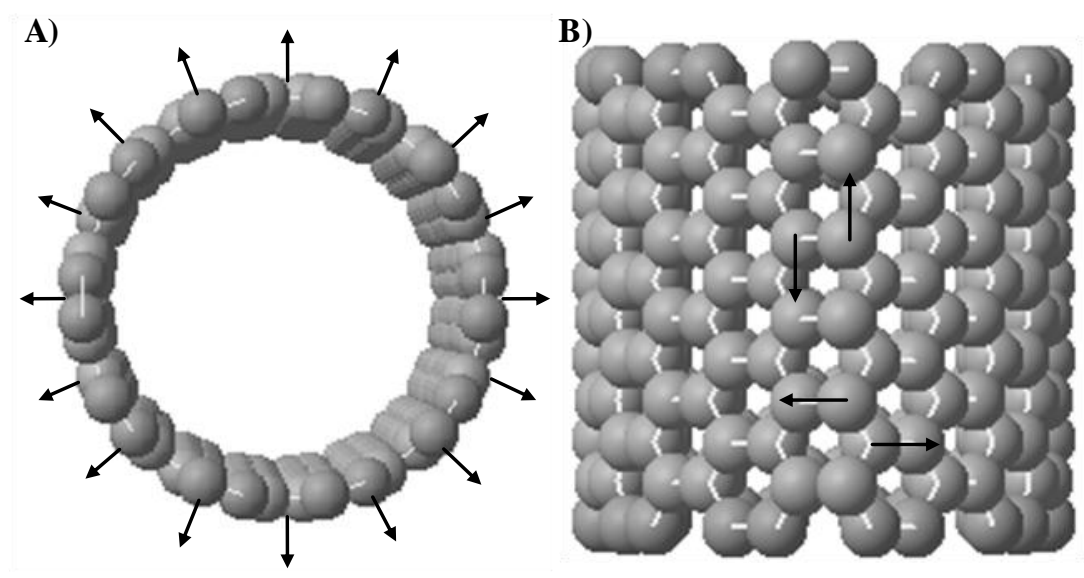

Figure 1-32: Raman active modes: A) The radial breathing mode and B) tangential stretching modes of the $\mathbf{G}$ band.

\section{Radial breathing modes (RBMs)}

Radial breathing modes are generally found at low energies, in the range of $100-$ $300 \mathrm{~cm}^{-1}$, and can be attributed to the circumferential pulsing of individual tubes, as shown in Figure 1-32A. The RBMs are an important diagnostic feature for the characterisation of CNT samples, firstly because they are only seen for SWNTs, whereas for MWNTs they are too weak to be observable. This means the existence of RBMs in a spectrum is almost certain proof of SWNTs in the given sample. Additionally, it has been shown that the frequency of the RBM $\left(\omega_{\mathrm{RBM}}\right)$ is highly dependent on the diameter of the tube. ${ }^{104}$ In fact, the relationship between diameter $\left(\mathrm{d}_{\mathrm{t}}\right.$, units of $\left.\mathrm{nm}\right)$ and $\omega_{\mathrm{RBM}}$ (units $\mathrm{cm}^{-1}$ ) appears quite simple and can be given by Equation 1-4.

$$
\omega_{\mathrm{RBM}}=\frac{\mathrm{A}}{\mathrm{d}_{\mathrm{t}}}+\mathrm{B}
$$

where $\mathrm{A}$ and $\mathrm{B}$ are experimentally determined parameters; $\mathrm{A}=234 \mathrm{~nm} \mathrm{~cm}{ }^{-1}$ and $\mathrm{B}=$ $10 \mathrm{~cm}^{-1}$ for bundles of tubes, ${ }^{105}$ and $\mathrm{A}=248 \mathrm{~nm} \mathrm{~cm}^{-1}$ and $\mathrm{B}=0 \mathrm{~cm}^{-1}$ for an isolated 
tube. ${ }^{106}$ For bundles of tubes, it was found that the frequency of the RBM was shifted with respect to individual tubes, as a result of tube-tube interactions. ${ }^{107}$ It should be noted that this relationship is not so useful for tubes with $d_{t}<1 \mathrm{~nm}$ because as the diameter decreases there is a resulting increase in $\mathrm{C}-\mathrm{C}$ bond distortion due to higher tube curvature and an increased dependence on nanotube chirality. ${ }^{108}$

\section{G band}

The other important feature in Raman spectra of carbon nanotubes is the G band, which can be found in the region of $1500-1600 \mathrm{~cm}^{-1}$. The $\mathrm{G}$ band is the combination of tangential $\mathrm{C}-\mathrm{C}$ vibrational modes of the graphene sheet so is observed for all forms of graphitic carbon. ${ }^{103}$ In CNTs, the symmetry of the graphene sheet is broken by effectively rolling the sheet into a tube, and so these stretching modes cause atomic displacement in two directions, both around the tube circumference and along the tube axis, summarised in Figure 1-32B.

In Raman spectra of graphite and MWNTs, the G band is often observed as a single broad peak at $\sim 1580 \mathrm{~cm}^{-1},{ }^{109}$ as in Figure 1-33. In SWNT samples however, up to six distinct peaks may be observed in the G band. ${ }^{109}$ This is because in SWNT samples, tube diameters and the diameter spread are usually much smaller than for MWNTs, where the generally large diameters and diameter spread leads to coalescence of the peaks into one broad signal. 


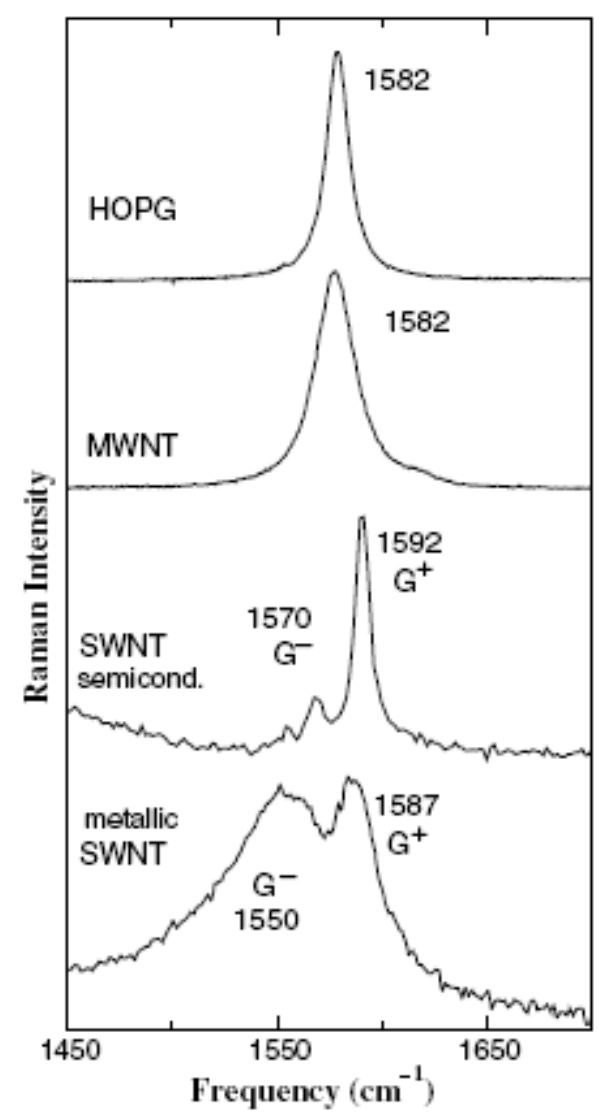

Figure 1-33: Comparative Raman spectra showing the tangential mode (G band) region for highly ordered pyrolitic graphite (HOPG), a MWNT bundle, an isolated semiconducting SWNT and an isolated metallic SWNT. ${ }^{110}\left(G^{+}\right.$refers to stretches along the tube axis while $G^{-}$refers to circumferential stretches)

\section{D band}

The $\mathrm{D}$ band is a common feature of carbonaceous materials including amorphous carbon, graphite and CNTs. It is normally found in the region of $1250-1450 \mathrm{~cm}^{-1}$ and is caused by the breakdown of the symmetry of the perfect $\mathrm{sp}^{2}$ graphene structure by the presence of defects in the nanotubes. As such, analysis of the ratio of the intensities of the $\mathrm{D}$ band versus the $\mathrm{G}$ band $\left(\frac{\mathrm{I}_{\mathrm{D}}}{\mathrm{I}_{\mathrm{G}}}\right)$ reveals the relative amounts of disordered and graphitic carbon, and thus provides a qualitative assessment of the structural disorder of CNT products. 
One potential pitfall with Raman spectroscopy is that it is not a quantitative method. When the energy of the incident laser approximately equals any transition gap in a given molecule - i.e. any electronic or vibrational transition - the Raman active modes for the molecule will experience an enhanced excitation and so will appear more intense in the Raman spectrum. This phenomenon is known as the resonance Raman effect. For CNTs, this means that for any nanotube with a band gap approximately equal to the energy of the incident laser, its corresponding Raman modes will appear more intense in the spectrum than a nanotube with a different band gap. Therefore, a strong signal in the Raman spectrum does not necessarily relate to a dominant species in the sample, but instead can be indicative of a nanotube transition energy close to the input energy. This effect is particularly relevant for the radial breathing modes, whose positions are highly diameter dependent. Figure 1-34 depicts a Kataura plot of the calculated transition energies of all possible combinations of SWNTs in the diameter range of $0.5<\mathrm{d}_{\mathrm{t}}<3.0 \mathrm{~nm}$. One can see that as diameter decreases, the transition energy increases. Nonetheless, this problem can be mitigated by using a range of excitation wavelengths. By changing the wavelength of the incident laser, one can enhance the resonance peak for different nanotubes. This can be particularly useful for examining nanotubes of different diameters and helicities to determine their electronic behaviour. 


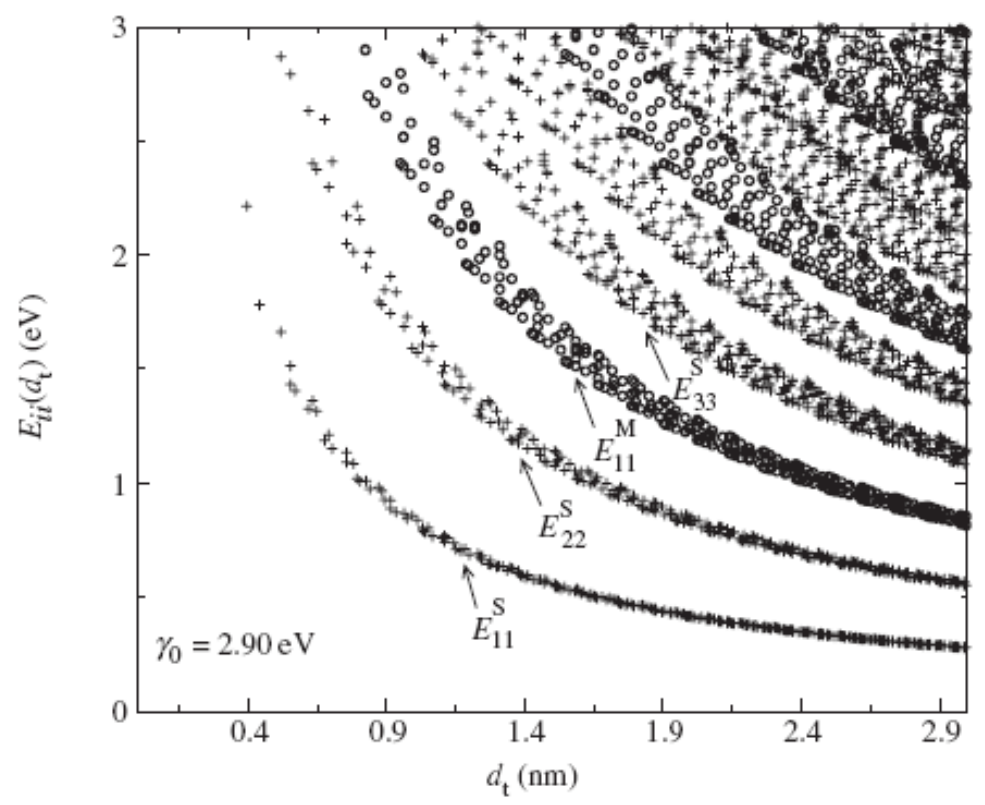

Figure 1-34: Plot of calculated transition energies, $E_{i i}$, of all possible (n,m) combinations as a function of tube diameter, $d_{t}$ (Kataura plot). ${ }^{27}$ Superscript $M$ and $S$ are used to describe metallic and semiconducting CNTs, respectively.

\subsection{Research Aims}

The first goal of this research was to investigate the growth of large CNT arrays using a gas phase CVD method, with a focus on the preferential growth of SWNTs. Different synthetic conditions and additives were used to encourage the growth of SWNTs.

The second aim of this research was to investigate the effect of the substrate on the growth of these large CNT arrays. Extended arrays on monolithic substrates are interesting for use in new functional devices for a wide range of applications. Understanding why some substrates allow the growth of large arrays of CNTs, while others hinder the growth, is a vital aspect in understanding the mechanism of CNT 
growth. A range of substrates were investigated - both conducting and nonconducting. 


\section{Experimental}

\subsection{Apparatus description}

A thermal chemical vapour deposition (CVD) process was employed to synthesise

CNTs. A schematic of the experimental apparatus is given in Figure 2-1. The apparatus consisted of a compressed gas cylinder linked to a flow controller to deliver an oxygen-free nitrogen carrier gas, and was subsequently coupled to a gas flow switch. The two outlets from the flow switch led onto two different paths that later converged. On one path, the gas flow fed directly to a reaction vessel, while the second path led to the reaction vessel via a solvent bubbler. This arrangement ensured that the inlet gas could be shifted from pure nitrogen carrier gas to a nitrogen gas/carbon source vapour mixture as necessary, by bubbling through a volatile carbonaceous solvent.

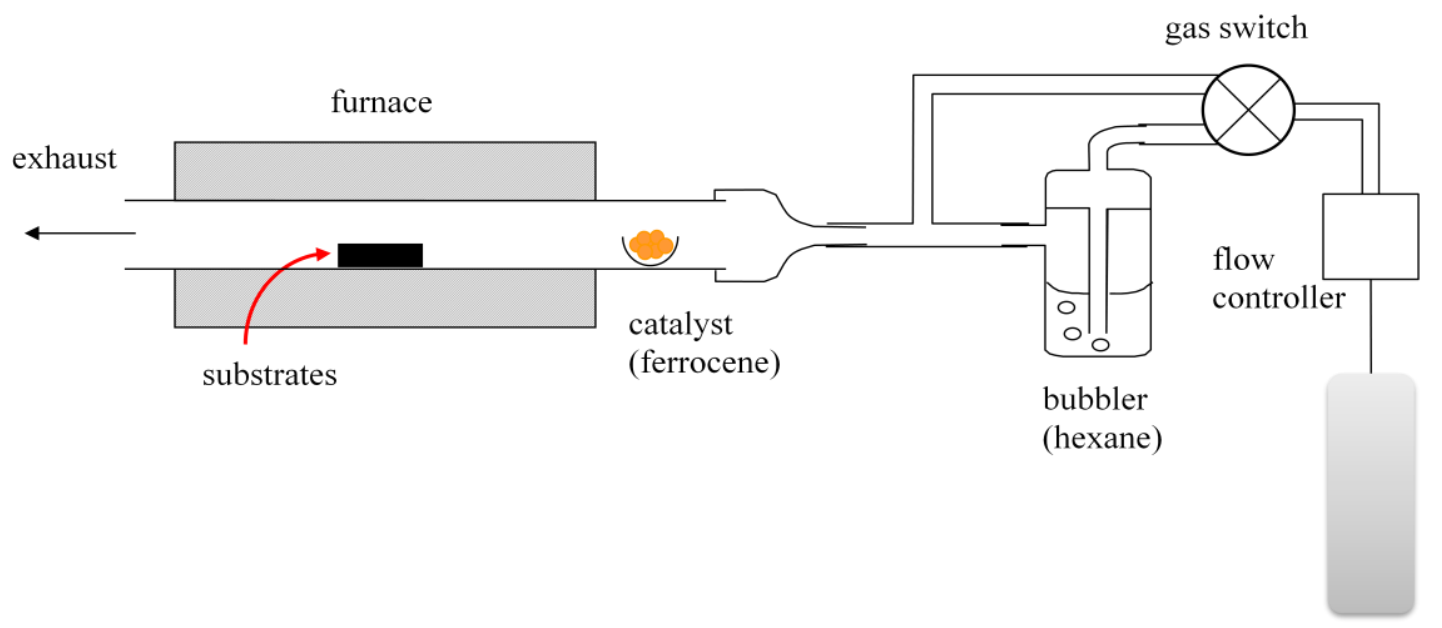

$\mathrm{N}_{2}$ cylinder

Figure 2-1: Schematic depicting the experimental apparatus used for chemical vapour deposition of CNTs. 
The reaction vessel used was a $25 \mathrm{~mm}$ (outer diameter) fused quartz reaction or working tube. The length of the working tube varied but was in the range of $0.8-$ $1.0 \mathrm{~m}$. The working tube was sealed and connected to the gas delivery system using purpose-built, tapered glass fittings with screw-on caps and silicone seals, depicted in Figure 2-2. Finally, the working tube exhaust outlet was connected to the laboratory exhaust system.

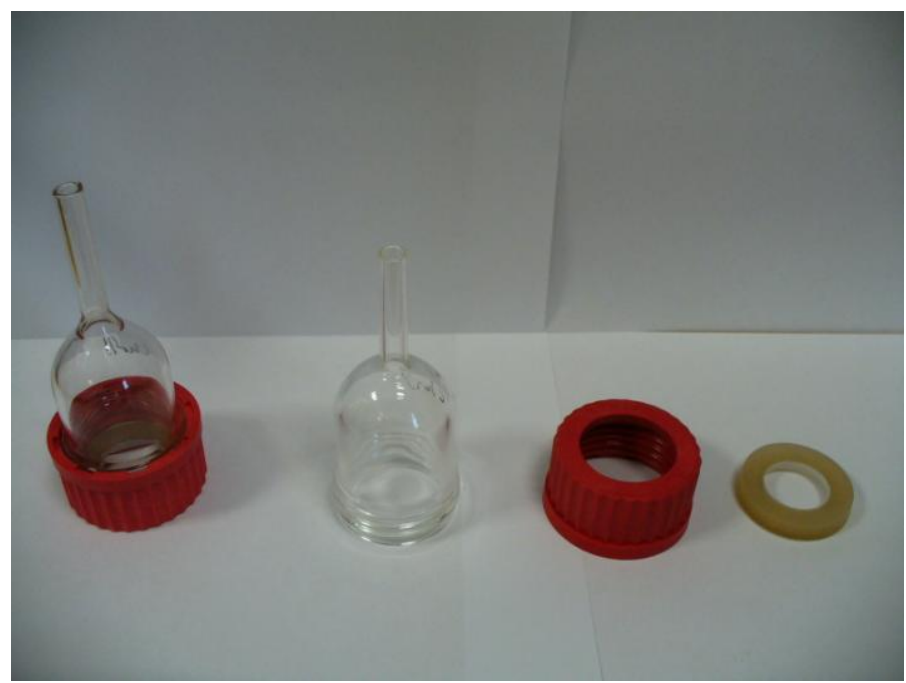

Figure 2-2: The purpose-built screw capped tapered glass fittings used to connect the working tube to the inlet and outlet gas flows.

High temperature reactions were carried out by placing the working tube inside a temperature-controlled, horizontal tube furnace. Two tube furnaces were used during the course of this work. The first was a $320 \mathrm{~mm}$ long, resistance wire-wound, horizontal, tube furnace with a $50 \mathrm{~mm}$ diameter inner cavity and an in-built temperature controller, which was constructed at Victoria University of Wellington. This furnace was later replaced by a Carbolite HST 12/-/400 horizontal, single zone, split tube furnace, purchased new from Total Lab Systems. The new furnace had a $400 \mathrm{~mm}$ heated length and $500 \mathrm{~mm}$ insulated length, with a $110 \mathrm{~mm}$ diameter 
internal cavity. It was controlled using a separate temperature controller box with a $1200{ }^{\circ} \mathrm{C}$ maximum operating temperature.

\subsection{Reaction furnace calibration}

Before carrying out experiments, the temperature controlled furnace was calibrated, both to relate the indicated furnace temperature with the measured temperature inside the quartz working tube and to determine the effective working width of the furnace. To accomplish this, a quartz reaction tube was placed in the furnace and connected to a nitrogen gas supply. The nitrogen flow rate through the reaction tube was set at approximately $110 \mathrm{~cm}^{3} \mathrm{~min}^{-1}$, and the furnace temperature was varied. At this point, the temperature inside the working tube was measured at different positions along the tube using an independent K-type thermocouple. The results of this calibration for the Carbolite furnace are given in Figure 2-3. 


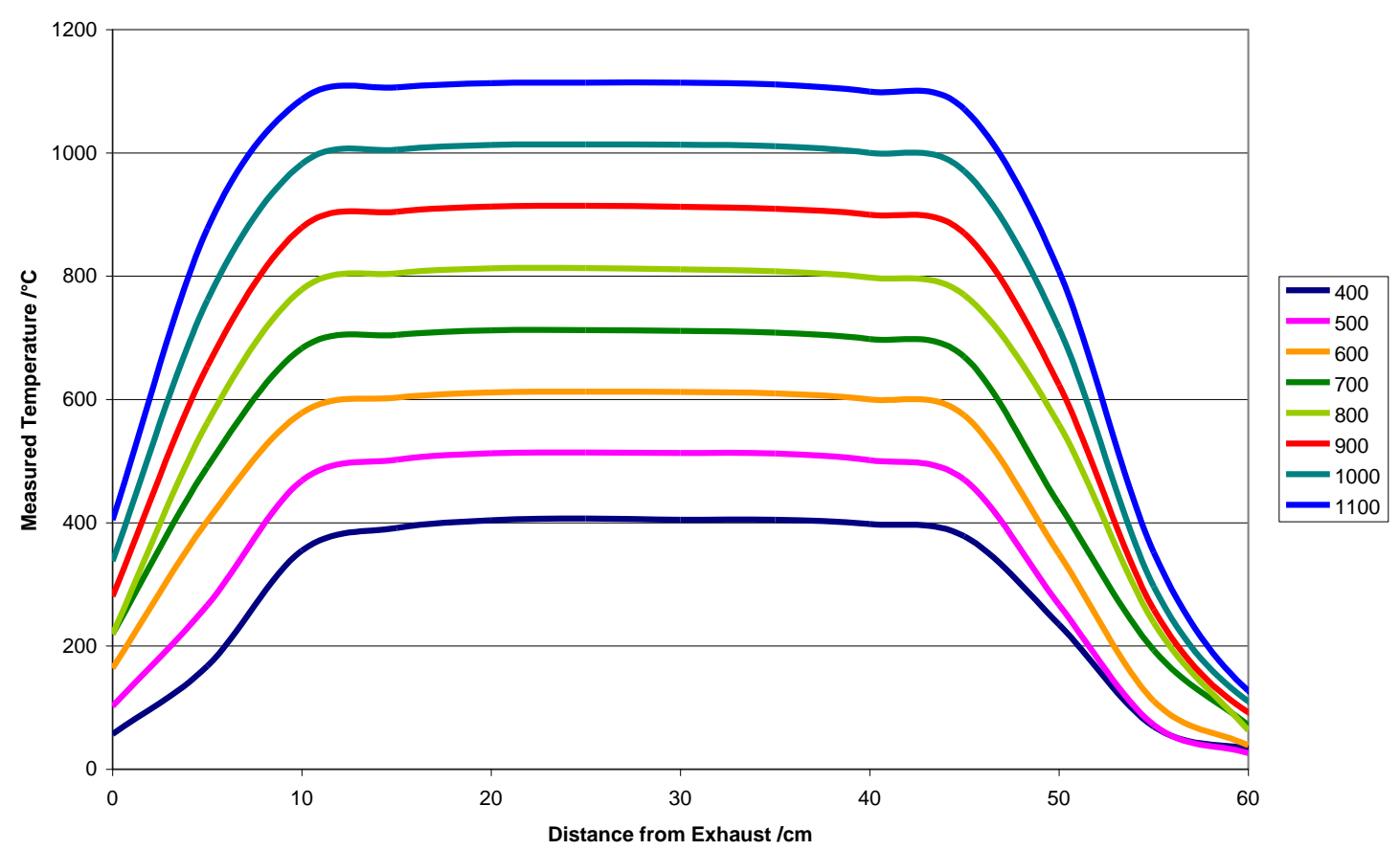

Figure 2-3: Furnace calibration graph indicating the range of the independently measured temperature in a quartz tube under nitrogen flow for various furnace readings. ${ }^{*}$

From the calibration data, it was found that the furnace output was reasonably well calibrated, with the measured temperature being roughly $1-2 \%$ higher than the set temperature. Also, it is clear that the furnace holds a constant temperature over a relatively large zone; $\pm 5^{\circ} \mathrm{C}$ over the $20 \mathrm{~cm}$ between the 15 and $35 \mathrm{~cm}$ marks. This region encompasses the centre of the furnace $(25 \mathrm{~cm})$ and is the hottest zone. This was considered the working area of the furnace and is where the substrates were placed.

\footnotetext{
\$Distance $(\mathrm{d} / \mathrm{cm})$ was measured from the mouth of the furnace at the exhaust end so that the inlet position of the furnace was at $50 \mathrm{~cm}$.
} 


\subsection{Chemical vapour deposition of carbon}

Hexane, ferrocene and sulfur were purchased from Sigma Aldrich and used without further purification. The cobaltocene employed had been synthesised previously within the group using a standard synthetic procedure outlined in Figure 2-4. ${ }^{111}$

$$
\begin{aligned}
\mathrm{Na}_{(\mathrm{s})}+2 \mathrm{C}_{5} \mathrm{H}_{6} & \longrightarrow 2 \mathrm{Na}\left(\mathrm{C}_{5} \mathrm{H}_{5}\right)+\mathrm{H}_{2} \\
{\left[\mathrm{Co}\left(\mathrm{NH}_{3}\right)_{6}\right] \mathrm{Cl}_{2}+2 \mathrm{Na}\left(\mathrm{C}_{5} \mathrm{H}_{5}\right) } & \longrightarrow \mathrm{Co}\left(\mathrm{C}_{5} \mathrm{H}_{5}\right)_{2}+2 \mathrm{NaCl}+6 \mathrm{NH}_{3}
\end{aligned}
$$

Figure 2-4: Reaction scheme for the preparation of cobaltocene.

Müller clusters were provided by Prof. Achim Müller from the Faculty of Chemistry at the University of Bielefeld, Germany. Two bimetallic clusters were employed during the course of this work: $\mathrm{Na}_{8} \mathrm{~K}_{14}(\mathrm{VO})_{2}\left[\left\{\mathrm{Mo}(\mathrm{Mo})_{5} \mathrm{O}_{21}\left(\mathrm{H}_{2} \mathrm{O}\right)_{3}\right\}_{10}\left\{\mathrm{Mo}(\mathrm{Mo})_{5} \mathrm{O}_{21}\left(\mathrm{H}_{2} \mathrm{O}\right)_{3}\left(\mathrm{SO}_{4}\right)\right\}_{2}\right.$ $\left.\left\{\mathrm{VO}\left(\mathrm{H}_{2} \mathrm{O}\right)\right\}_{20}\{\mathrm{VO}\}_{10}\left(\left\{\mathrm{KSO}_{4}\right\}_{5}\right)_{2}\right] \cdot c a 150 \mathrm{H}_{2} \mathrm{O}$, which will be referred to as $\mathrm{Mo}_{72} \mathrm{~V}_{30}$ from now on, and $\left[\left\{\mathrm{Na}\left(\mathrm{H}_{2} \mathrm{O}\right)_{12}\right\} \subset\left\{\mathrm{Mo}^{\mathrm{VI}}{ }_{72} \mathrm{Cr}^{\mathrm{III}}{ }_{30} \mathrm{O}_{252}\left(\mathrm{CH}_{3} \mathrm{COO}\right)_{19}\left(\mathrm{H}_{2} \mathrm{O}\right)_{94}\right\}\right]$ - ca $120 \mathrm{H}_{2} \mathrm{O}$, which is abbreviated to $\mathrm{Mo}_{72} \mathrm{Cr}_{30}$. These were dissolved in distilled water and the solutions $(0.36 \mathrm{mM})$ were drop cast onto silicon wafer substrates. The aqueous solution of $\mathrm{Mo}_{72} \mathrm{~V}_{30}$ is air sensitive so was stored and used under nitrogen.

Quartz substrates were purchased from GM and Associates and highly ordered pyrolitic graphite (HOPG) was obtained from Wale Apparatus Company. Samples of glazed porcelain, alumina, Pythagoras, silicon wafer, $\beta$ - and $\mathrm{O}^{\prime}$-sialon were obtained from Dr. D. V. White at Industrial Research Ltd (IRL). Glassy carbon and sapphire were obtained from SPI Supplies and University Wafer, respectively. Samples of two iron-nickel-silicon alloys, MBF20 and MBF30 were obtained from Metglas. Substrates were first cleaned by washing with water and then acetone. To 
keep the surface morphologies as consistent across substrates as possible, rougher samples were sent to Stewart Bush in the School of Geography, Environment and Earth Sciences for polishing. Using a Struers DP-U2 polishing unit operating at 300 rev min $^{-1}$, polishing was accomplished using a PAD-K cloth and employing in succession 6, 3, and $1 \mu \mathrm{m}$ WP diamond suspensions as the abrasive. Water was used as a lubricant.

Chemical vapour deposition of CNTs was performed using a thermal decomposition method in combination with a floating catalyst. A typical experiment was conducted by placing a ceramic boat with a measured amount of ferrocene catalyst inside the working tube at the entrance of the furnace where the temperature was found to reach approximately $180{ }^{\circ} \mathrm{C}$, independent of the furnace temperature setting. Hence, the position of the boat was altered according to the temperature of any given reaction, to ensure that the catalyst remained at $180{ }^{\circ} \mathrm{C}$, as determined by prior furnace calibration. This temperature was just sufficient to cause the ferrocene to sublime slowly. Experiments were also attempted where the catalyst was independently heated by a separate furnace, however problems with reproducibility of the catalyst sublimation system meant that the experimental results from this are not considered here.

Substrates were positioned in the central zone of the furnace and the bubbler was filled with hexane. Nitrogen gas flow was then set to $110 \mathrm{~cm}^{3} \mathrm{~min}^{-1}$ prior to heating the furnace to $800{ }^{\circ} \mathrm{C}$. Once the furnace temperature had settled and the catalyst 
began to sublime, the carbon source was introduced by switching the nitrogen flow through the solvent bubbler. The carbon source used here was hexane and it was introduced for 20 min before switching back to pure nitrogen flow and allowing the furnace to cool.

Once cool, the substrates were recovered and scrapings of the deposits lining the inside walls of the working tube were taken for analysis.

\subsection{Characterisation}

After CNT deposition, substrates and scrapings from the quartz tube walls were analysed using a variety of characterisation techniques.

Scanning Electron Microscopy (SEM) was performed using a JEOL 6500F field emission scanning electron microscope with the accompanying JED-2300T EDS detector for Energy Dispersive Spectroscopy (EDS). Generally, the SEM was operated at $15 \mathrm{kV}$, although this was adjusted as required in order to optimise the viewing conditions for different substrates and sample conditions. Sample preparation involved mounting the sample on an aluminium or brass stub using double-sided carbon tape. No further preparation was needed, as the samples were usually conductive enough to avoid significant charging effects.

Transmission Electron Microscopy (TEM) was carried out using a JEOL 2010 high resolution transmission electron microscope at an accelerating voltage of $200 \mathrm{kV}$. Samples were prepared by suspending CNTs in THF or toluene by sonication. This meant either using tube scrapings or scraping a small amount of CNTs from a 
substrate surface. A drop of the CNT suspension was then placed onto a copper sample grid covered with a holey carbon film, and allowed to dry.

Raman spectra were obtained using a Jobin Yvon LabRam confocal Raman spectrometer with x50 long working distance objective coupled to a BX41 Olympus microscope system. Samples were studied as synthesised in the solid state. For CNTs grown on substrates; the substrate was placed directly under the laser beam, while tube scrapings were first placed onto a glass microscope slide, which was then placed under the laser beam. Spectra were measured between 150 and $3000 \mathrm{~cm}^{-1}$ by irradiating with a $633 \mathrm{~nm}$ He-Ne laser.

Thermogravimetric Analysis (TGA) was conducted using a Shimadzu TGA-50 apparatus. Samples were analysed as synthesised by loading a small quantity onto a platinum pan - between 2 and $5 \mathrm{mg}$ - which was in turn placed on the balance inside the furnace of the apparatus. The furnace was closed and the sample was heated to $900{ }^{\circ} \mathrm{C}$ at a ramp rate of $10{ }^{\circ} \mathrm{C} \min ^{-1}$ under a dry air flow $\left(20 \mathrm{~cm}^{3} \mathrm{~min}^{-1}\right)$. 


\section{Control Experiments}

This chapter deals with the results from the studies of the standard synthetic conditions, including analysis of the effect of different parameters and species on the deposited products.

\subsection{Initial Results}

The standard synthetic procedure was carried out at $800^{\circ} \mathrm{C}$ as described in Section 2.3, using ferrocene and hexane as the active species. Because a floating catalyst was used, after CVD there were large black deposits covering all inside surfaces of the working tube, over the full heated length of the tube. Scrapings from different regions were taken and then mounted onto an SEM stub for further analysis.

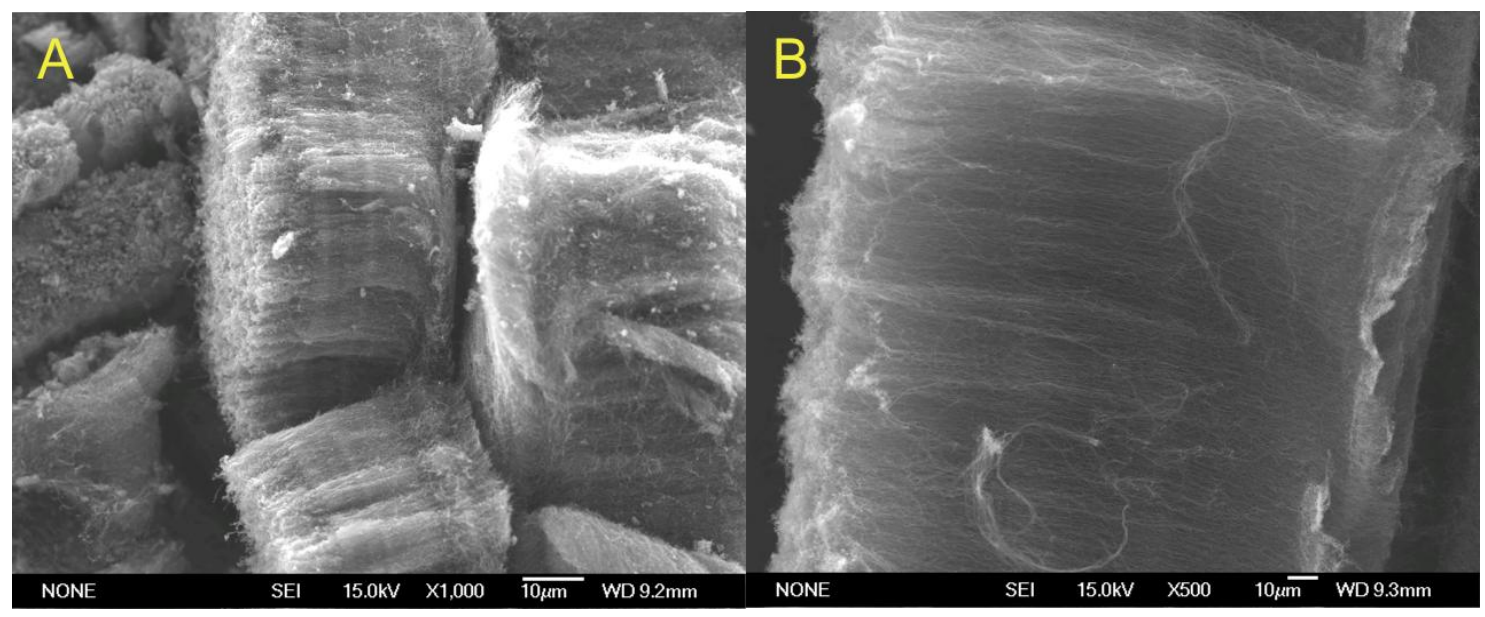

Figure 3-1: SEM image comparing CNT deposits from A) the early region of the working tube (approx. 0-15 cm), before the hot zone and B) later in the tube (>15 cm), within the hot zone and beyond.

Figure 3-1 illustrates the difference between the deposits from the earlier regions of the tube - from the entrance of the furnace to approximately $15 \mathrm{~cm}$ inside the heated 
region - and those deposits from later in the tube, including from within the hottest region of the furnace. In Figure 3-1A one can see deposits from the early part of the tube. Here, it was found that the CNT fields tended to be shorter, curlier, and were often surrounded or interspersed with a lot of visible amorphous carbonaceous material and excess catalyst. This meant that the fields appeared much dirtier than the deposits found later in the furnace, such as in Figure 3-1B. These regions appeared significantly cleaner, with almost no visible amorphous carbon or catalyst material. These differences are even more apparent in Figure 3-2. In Figure 3-2A one can see a deposit of CNTs that appear to have lots of small nodules at different points along their lengths. From the matching RBS image in Figure 3-2B, these small nodules can be seen as lots of small bright patches and are small iron catalyst particles. 


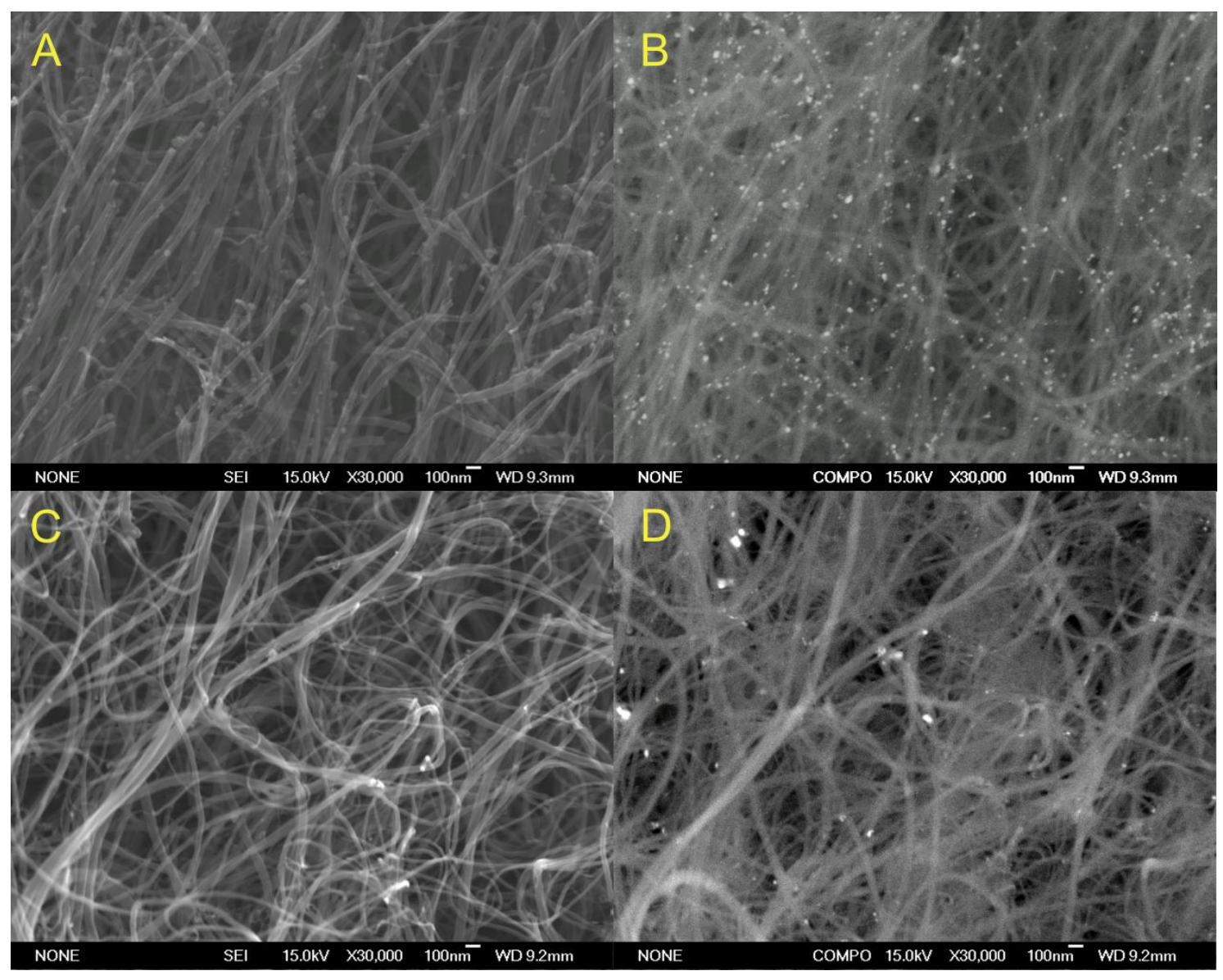

Figure 3-2: Comparative SEM images of the carbon deposits from different regions in the tube. A) SEI image of a deposit from early in the tube and B) the corresponding RBS image, while C) depicts an SEI image of a deposit from within the hot zone with D) its corresponding RBS image.

In contrast, the deposits seen in Figure 3-2C \& D are largely free of these residual iron particles. This is not surprising as once the decomposition of ferrocene occurs at high temperature, the transport of metallic iron species along the tube would be significantly reduced, leading to an accumulation of catalyst particles in the earlier regions. The reason for the marked disparity in the graphitisation of the carbon products is due to the relative temperature of the environment at different points within the working tube. In the early part of the furnace the gases were much cooler than in the central, hot zone, favouring the deposition of amorphous carbon. However, the higher temperatures within the central zone favour the deposition of 
more ordered carbon products, so CNT growth here and in the later regions was much faster and cleaner.

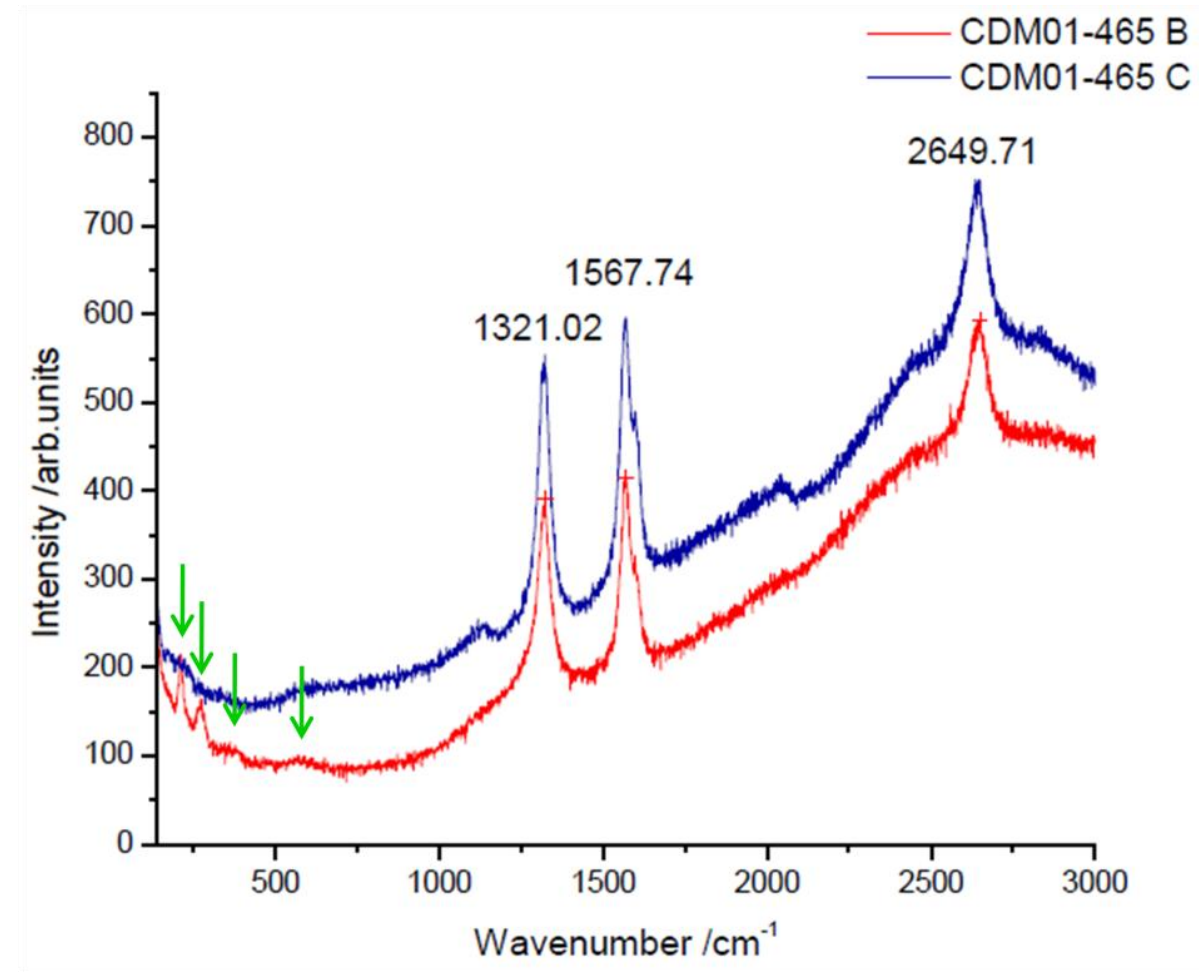

Figure 3-3: Comparative Raman spectra of CNT deposits from different regions of the working tube. Note the four low peaks in the $150-600 \mathrm{~cm}^{-1}$ region, evidence of iron oxide impurities in the Region $B$ sample from earlier in the tube.

This regional difference is also apparent in the observed Raman spectra, depicted in Figure 3-3. The red spectrum corresponds to a deposit from early in the tube, while the blue spectrum is from the central zone. Both have the expected peaks for the D and $\mathrm{G}$ bands $-1328 \mathrm{~cm}^{-1}$ and $1578 \mathrm{~cm}^{-1}$ respectively - though the ratio between the two $\left(\frac{I_{D}}{I_{G}}\right)$ is better for the blue spectrum ( 0.59 versus 0.90 for the red spectrum). This suggests a higher degree of graphitisation in the products from the central zone due to the presence of fewer amorphous carbon deposits and defects within the tubes. In addition, in the red spectrum there are four extra bands at 218, 285, 392 and 
$592 \mathrm{~cm}^{-1}$ that are not apparent for the blue spectrum. These peaks indicate the presence of significant magnetite nanoparticle impurities, consistent with a higher level of impurities in the early region. ${ }^{112}$

Figure 3-4A depicts a low magnification (x100) SEI image of a typical selected region of products deposited onto the side walls of the central, hot zone of the quartz reactor tube. One can see large, dense fields of what appear to be vertically aligned filamentous deposits. These aligned fields of CNTs from the central region of the working tube are relatively free of impurities and so from this point on, all results will focus on the products from this central region. As a result of the scraping and mounting processes, the fields lost some of their alignment and became randomly oriented on the SEM stub. In the right-hand side of the image one can see the flat face of a dense field of aligned CNTs. Enlarged in Figure 3-4C, this corresponds to the bottom face that was directly attached to the reactor wall and so its growth direction was constrained. As the reactor is made of fused quartz and the walls are relatively smooth, this is reflected in the growth of a smooth lower face on the CNT field. Conversely, on the left-hand side of the image one can see the rougher top face of a CNT field, which is further enlarged in Figure 3-4B. While this face was uninhibited by the side walls after the initial growth period, it was interesting to note that the fields tended to grow to a common length and so the top face still appears regular. 


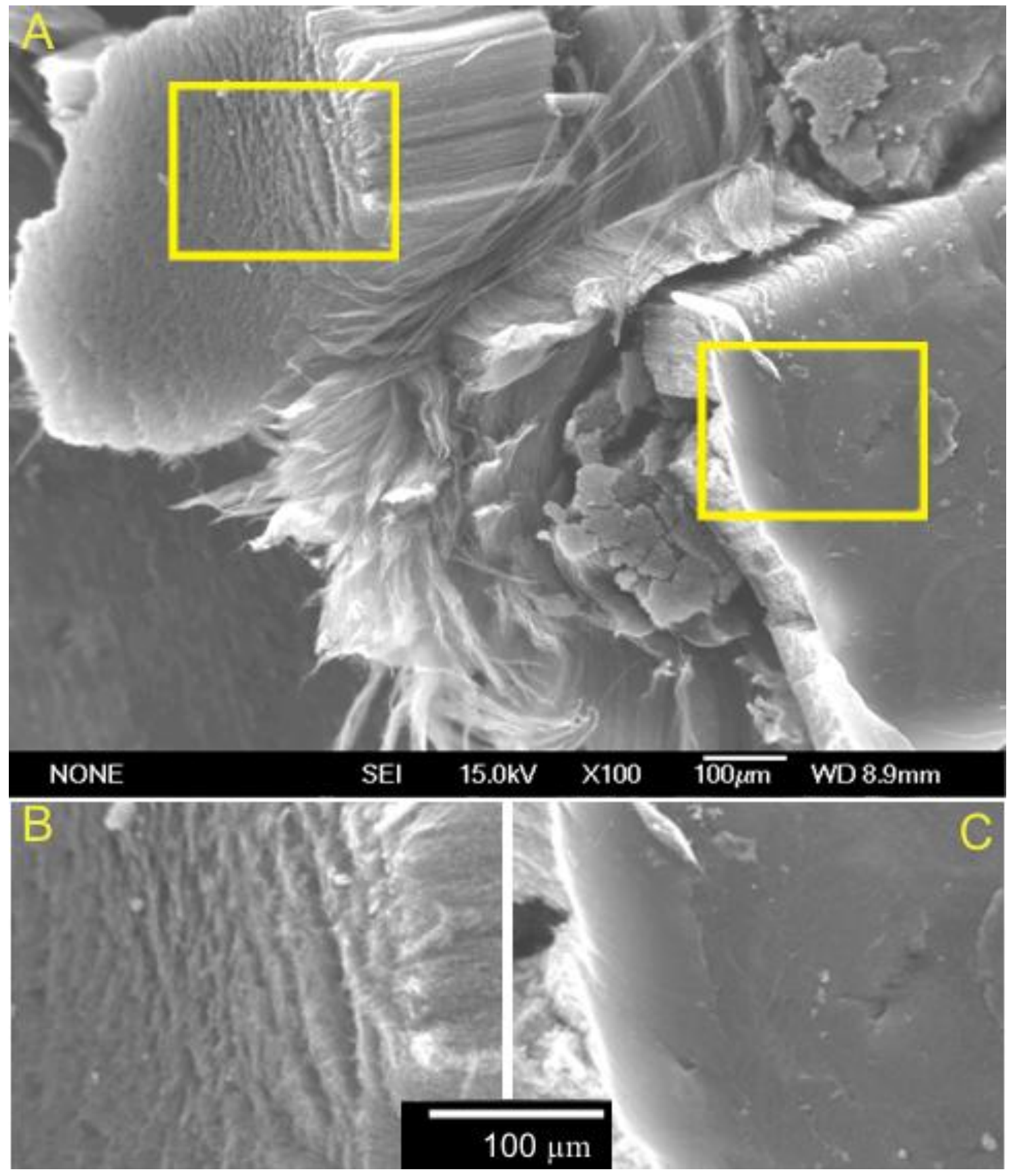

Figure 3-4: A) Low magnification SEM image of products from reactor sidewalls after CNT deposition. B) and C) are enlarged views of the indicated regions.

Further evidence of this field regularity is revealed in Figure 3-5. Here one can see a side-on view of a dense field of CNTs, which appears very uniform due to the highly consistent growth of the constituent fibres. This field regularity was a common feature of all successful experiments; however field height between individual trials 
varied greatly. Naturally, field height is a function of the amount of carbon added to the system and catalyst activity. Theoretically it should be possible to grow CNT fields with indefinite length by continuously feeding a carbon source into the system. However, in reality after some time the growth of a CNT field is greatly limited by the deactivation of the catalyst and, for a base growth mechanism, diffusion of the reactants through the already-grown nanotube field to the catalytic site.

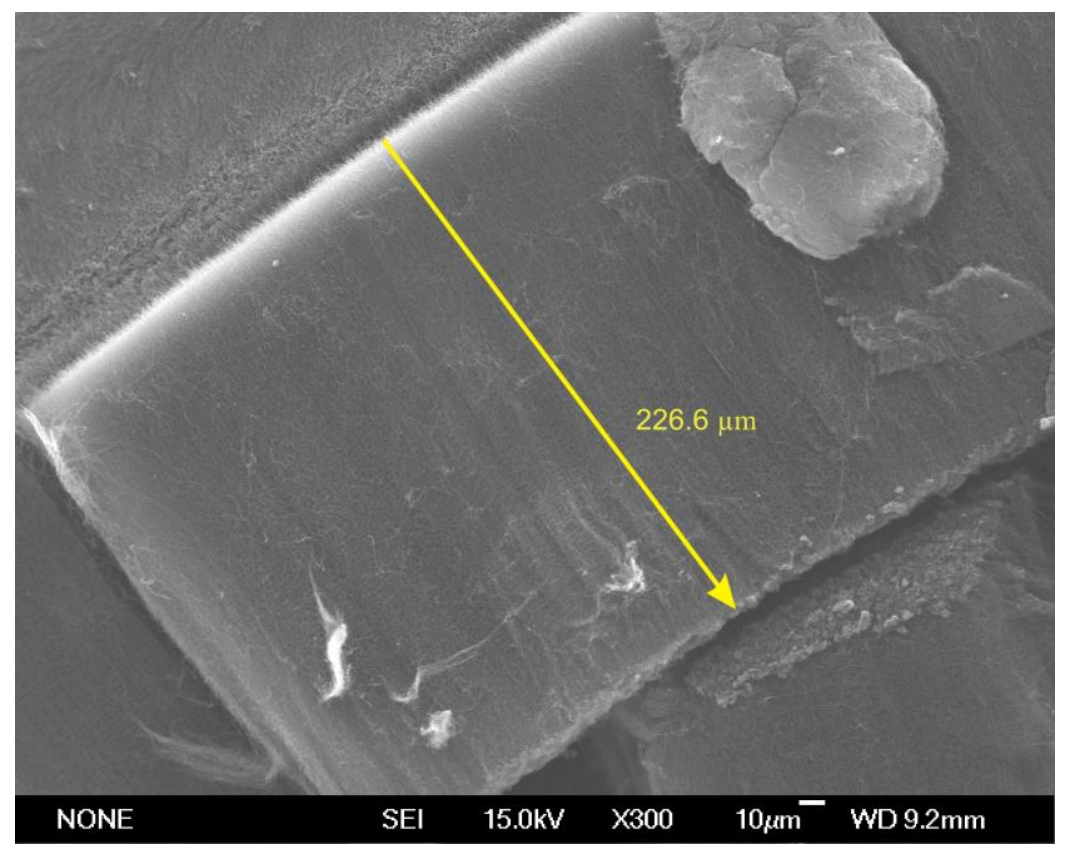

Figure 3-5: Cross-sectional view of an aligned field of CNTs.

There are two main causes of catalyst degradation in CNT growth: formation of an encapsulating carbon layer and incorporation into the supporting material. ${ }^{92,113-115}$ The length of time that the catalyst remains active is strongly dependent on the reaction conditions, particularly on the reaction temperature. While high temperatures are needed in order to obtain highly graphitised products, as temperature increases the decomposition of the carbon precursor and catalyst 
diffusivity increase also. This excess of active carbon species can lead to rapid deactivation due to carbon encapsulation of catalyst particles, while the faster diffusion can cause the catalyst to absorb into the supporting layer. Thus there is a temperature window for optimum growth of CNTs, which balances these opposing forces. $^{115}$

Diffusion-limited growth of carbon nanotubes is a much more difficult phenomenon to investigate. To accomplish this, an examination of the growth kinetics involving many CNT growth reactions under different conditions and for different lengths of time is often needed. Alternatively, the kinetics can be studied by in situ analysis of the growth using microscopy or laser reflectivity. ${ }^{86,116}$ Using either method, the growth rates are examined over time to determine the kinetic mechanisms of growth and growth termination.

In the present studies, the characterisation tools required for intense examination of the growth kinetics were unavailable, so the reaction time was kept constant at 20 min, which is too short for catalyst deactivation - due to encapsulation - and diffusion-limited growth to become a significant issue, according to similar published studies. ${ }^{86}$ In the successful experiments, the field height was generally found to be within the range of $100-250 \mu \mathrm{m}$.

If one looks closely at the carbon fields, one can see the individual constituent CNTs, as illustrated in Figure 3-6. Figure 3-6A is an SEM image of several nanotubes from within a single field that range in diameter from about $25-80 \mathrm{~nm}$. One can see that many of the nanotubes are not straight, indicating a degree of 
disorder and the presence of point defects in the tube structure. The large diameter range and the fact that many were not straight also meant the packing of the carbon filaments was seemingly erratic. While it is not possible to deduce for certain from an SEM image whether the products are graphitic - and if so, whether they are single or multiwalled - from the observed diameters the tubes were almost certain to be MWNTs. This postulation was confirmed by TEM, with Figure 3-6B depicting a $25 \mathrm{~nm}$ MWNT with approximately 25 walls. However, the as-grown MWNTs also had a thin coating of amorphous carbon $(\sim 2 \mathrm{~nm})$ and in some cases there were small catalyst particles trapped within the tubes.
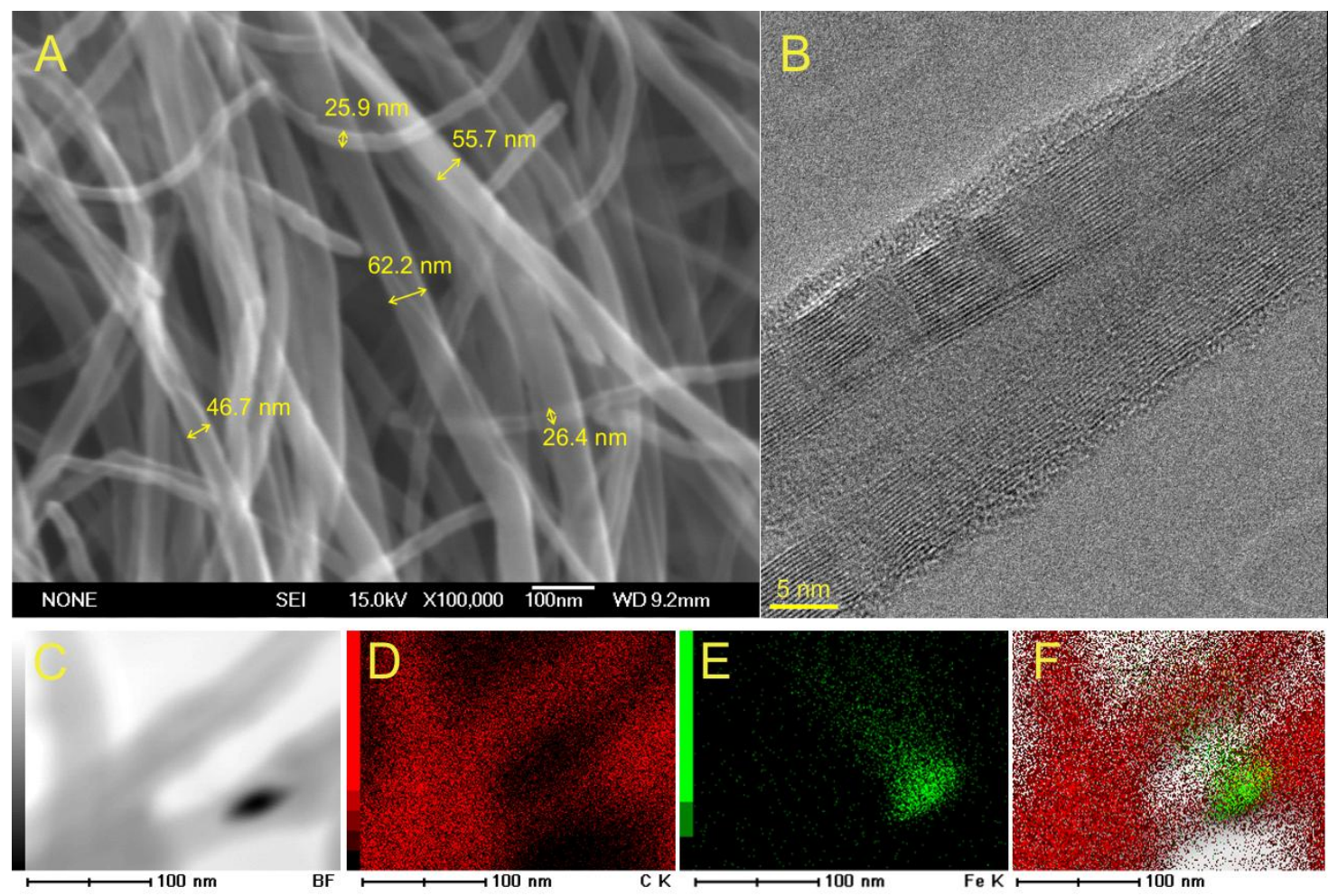

Figure 3-6: A) High magnification SEM image of as-grown CNTs and B) a TEM image of an individual MWNT. C - F) STEM EDS maps depicting an iron particle within the walls of a MWNT. 
Thermal analysis of samples was performed in order to determine the purity of the aligned MWNT fields and is illustrated in Figure 3-7. When burnt under a flow of air, two distinct regions of mass loss were observed. The first, between approximately 360 and $370{ }^{\circ} \mathrm{C}$, accounted for about $3 \%$ of the total mass and is consistent with the oxidation of amorphous carbon deposits such as those seen on the MWNT in Figure 3-6B. A second larger region between 585 and $770{ }^{\circ} \mathrm{C}$ accounted for approximately $95 \%$ of the total mass loss. This relates to the total destruction of the CNT deposits and the onset temperature is consistent with the oxidation of MWNTs. This left roughly $2-3 \%$ of the total mass remaining, which slowly increased with time and can be attributed to the oxidation of the residual iron catalyst. Therefore, while TEM appeared to show a significant amount of impurities, these account for only $4-10 \%$ of the products.

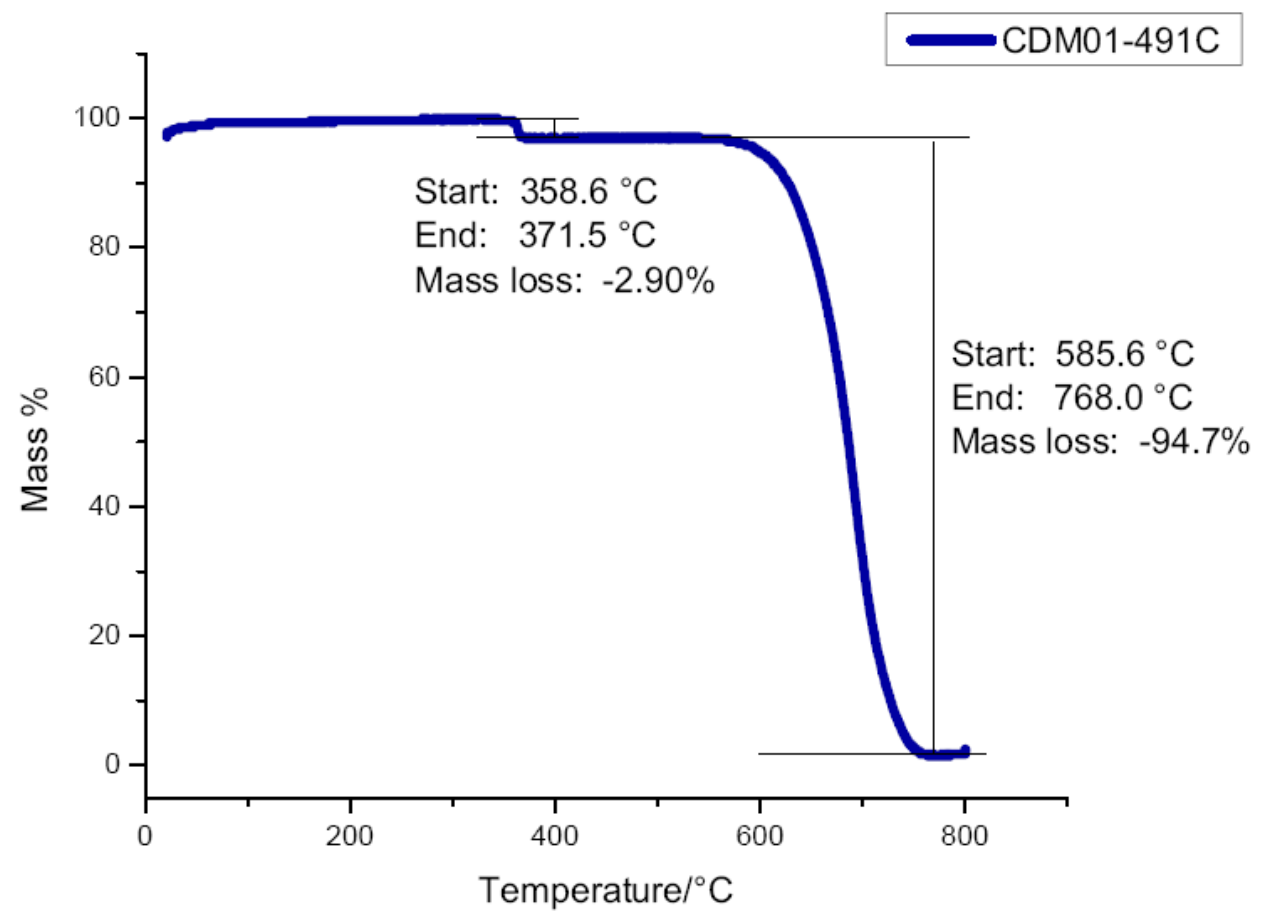

Figure 3-7: Plot of TGA results of a pristine CNT array revealing two discrete regions of mass loss. 
The TGA results indicate that the MWNTs are very resistant to oxidation below 580 ${ }^{\circ} \mathrm{C}$, thus it should be possible to burn off the amorphous carbon at $500{ }^{\circ} \mathrm{C}$ in air, leaving the MWNTs unaffected. This idea was tested by heating a sample for 30 min under these conditions. Figure 3-8 demonstrates the effect of controlled oxidation at $500{ }^{\circ} \mathrm{C}$. Comparisons between the TEM images in Figure 3-8A and Figure $3-8 \mathrm{~B}$ show that heating in air removed the amorphous carbon deposits on the surface of the MWNTs, while leaving the nanotubes themselves apparently intact. Additionally, the SEI image in Figure 3-8C reveals several brighter regions on the CNT fields. Comparison with the EDS maps in Figure 3-8D and E illustrates that these regions have higher levels of iron and much less carbon than the surrounding areas. This suggests that the amorphous carbon surrounding the excess iron catalyst was eliminated by oxidation, so that the iron residues were much more visible than previously. 

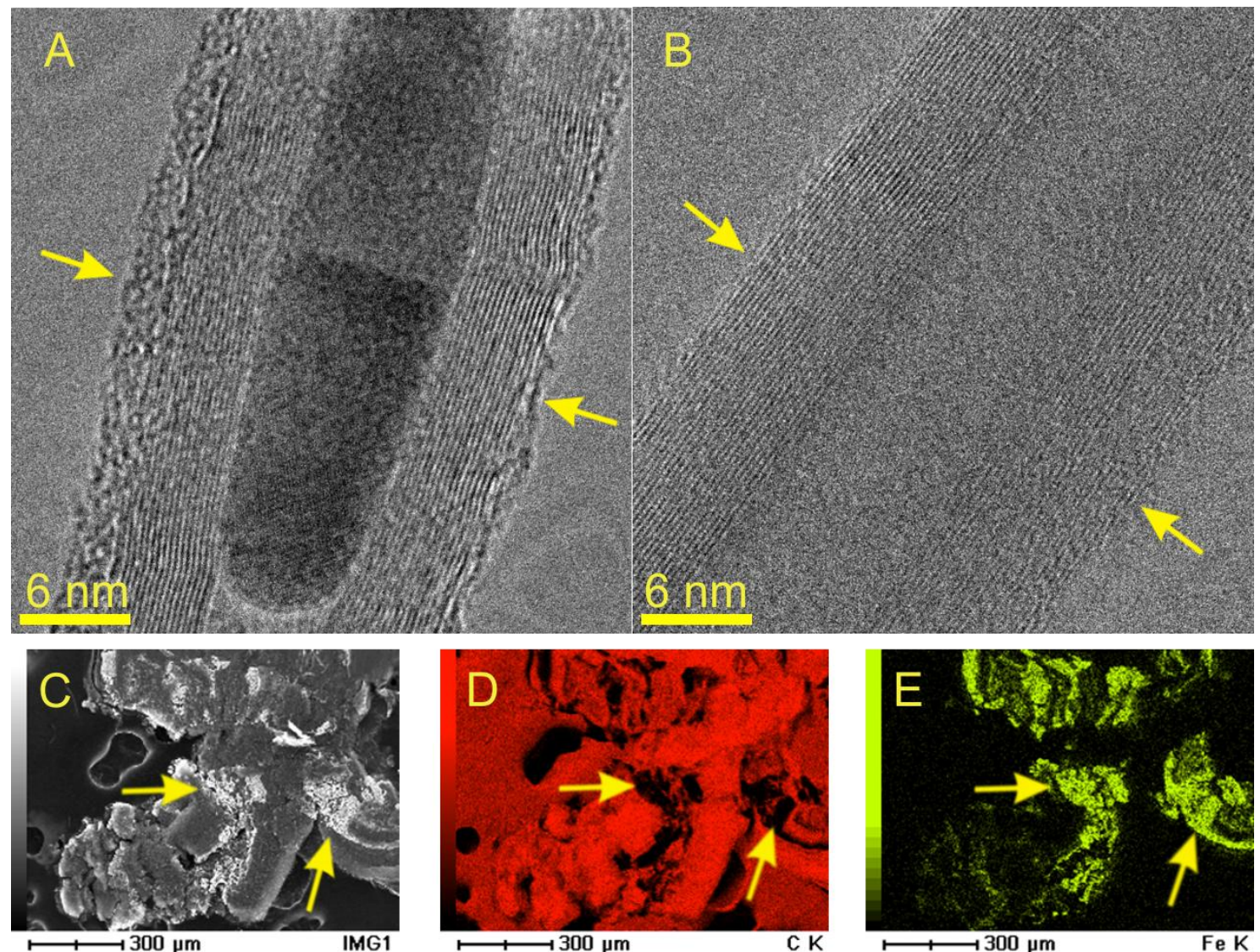

Figure 3-8: Comparative TEM images between A) an as-grown MWNT and B) after 30 min heating at $500{ }^{\circ} \mathrm{C}$ in air. There is a noticeable decrease in the amount of amorphous carbon. $\mathrm{C}-\mathrm{E}$ ) EDS maps illustrating the removal of carbonaceous material encapsulating the iron catalyst.

As mentioned above, the deposited MWNTs were quite haphazard, with a large range of diameters and seemingly random orientation. What was interesting however was that these apparently random tubes formed well aligned, extended fields with a striking wave-like pattern, demonstrated in Figure 3-9. This pattern is often observed in CNT fields and is likely caused by the dense packing of CNTs with different diameters. Of course, because the CNTs are randomly sized and orientated, these arrays would not be useful for some applications. Ideally, one wants an aligned array of identical, straight CNTs so that their observed properties are also identical, though this is still one of the greatest challenges of CNT synthesis. 


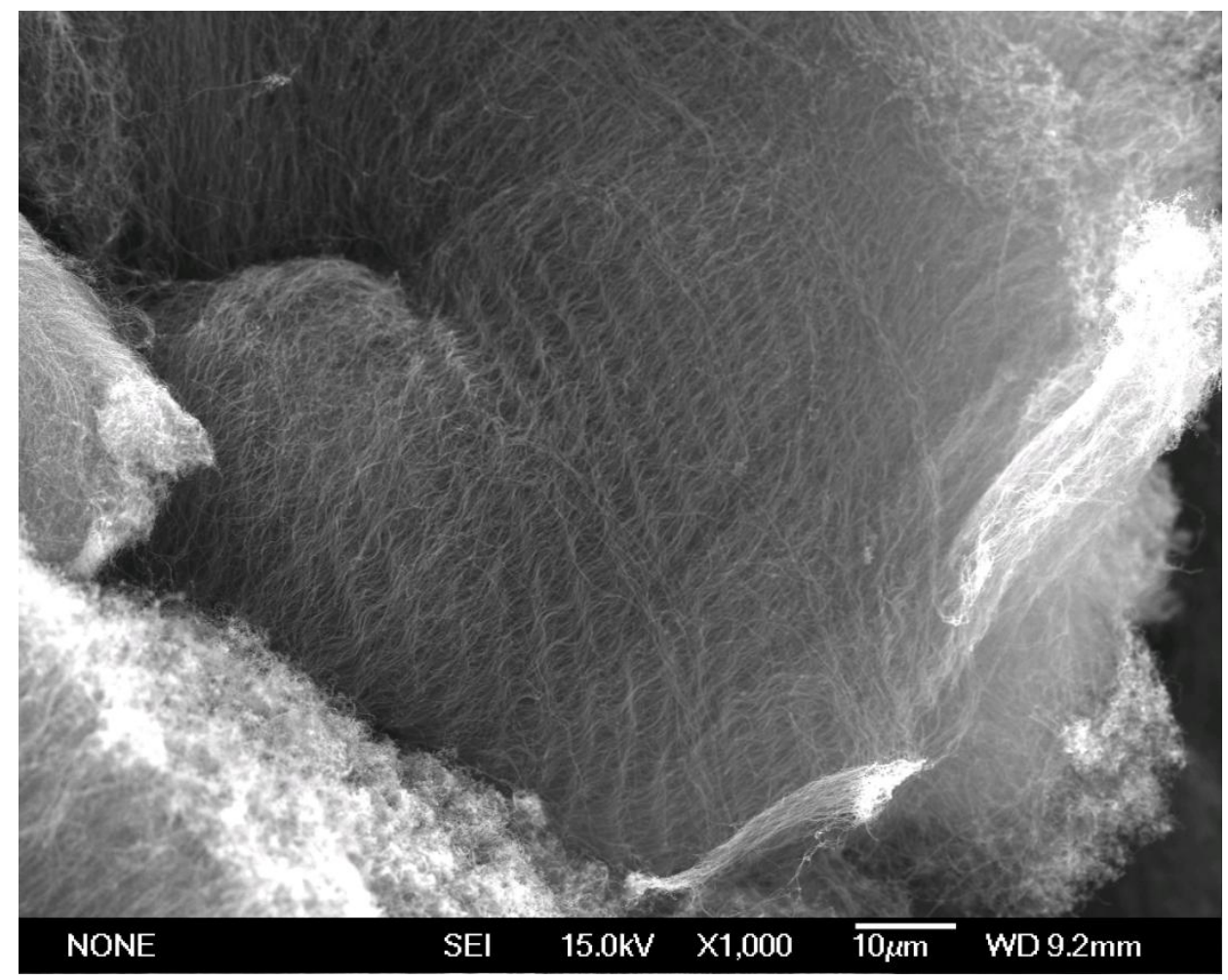

Figure 3-9: SEM image of a pristine, as-grown CNT field exhibiting a wave-like pattern of the CNTs.

This problem of controlling the individual nanotube morphologies is demonstrated by the results of one particular experiment that are depicted in Figure 3-10. In Figure 3-10A, one can see a field of aligned CNTs, which plainly shows two discrete growth domains. The enlarged image in Figure 3-10B shows that the CNTs in the lower part of the image are aligned with the same wave pattern seen previously. However, the CNTs in the upper part of the image appear much straighter. This must have been a result of a change in the growth conditions as the reaction progressed. The real issue is that there was no intended change in the conditions during this reaction; no apparent change in the input of carbon precursor or catalyst, nor in the temperature. This result was not reproducible and clearly illustrates the need for careful control of all synthetic parameters when attempting to grow CNTs. 


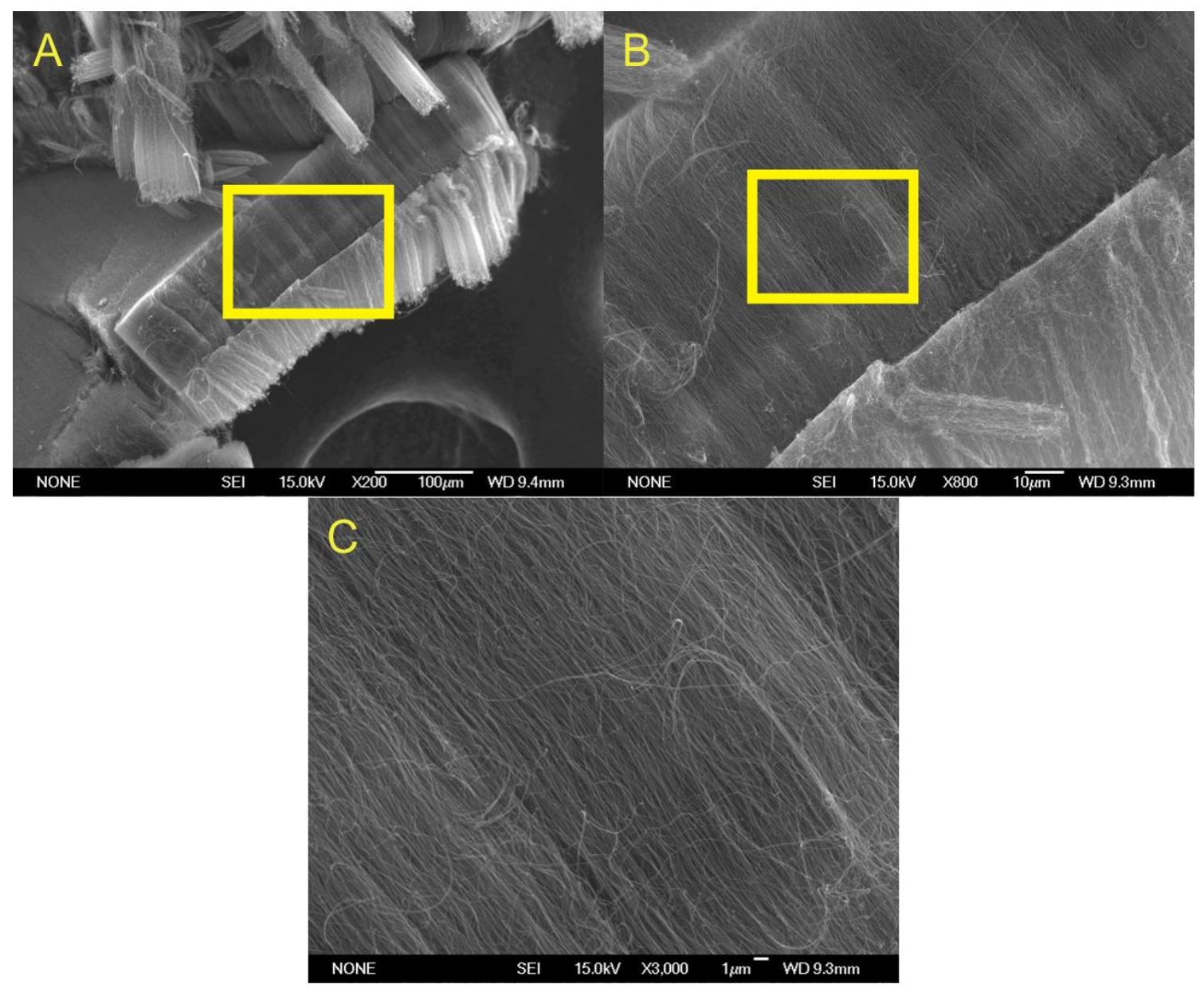

Figure 3-10: A - C) Series of SEM images of increasing magnification, depicting a field of CNTs with two discrete growth domains.

\subsection{Effect of reaction temperature}

As shown, initial tests produced large, dense, aligned fields of MWNTs. However, these results were only preliminary and did not necessarily correspond to optimum growth conditions. Furthermore, the aim of this research was to produce SWNT arrays employing a floating catalyst method and the decomposition of hexane. To determine the ideal conditions for the growth of CNTs under these conditions, several reaction parameters were varied to test their effect. Firstly, the effect of 
reaction temperature was investigated. Previous accounts describe a wide range of temperatures for the growth of CNTs, anywhere between 600 and $1100{ }^{\circ} \mathrm{C} .{ }^{115,117-121}$ To find the ideal reaction temperature for this system, additional reactions were performed at 700 and $900{ }^{\circ} \mathrm{C}$. The results from these investigations are depicted in Figure 3-11. At $700{ }^{\circ} \mathrm{C}$, the carbon products did not look like CNTs at all, looking far more like agglomerates of curly and disordered filaments. On the other hand, the products from the reaction at $900{ }^{\circ} \mathrm{C}$ showed evidence of fields of MWNTs. However these fields exhibited significant levels of impurities, such as amorphous steel-like films. This suggested the hexane decomposition rate is much higher than the rate of diffusion of the active carbon species and subsequent CNT growth. Hence, the carbon excess is deposited as carbon films rather than as nanotubes. Unfortunately, the furnace used in these experiments did not allow for finer temperature control within these ranges, therefore, it was decided that $800{ }^{\circ} \mathrm{C}$ was the preferred temperature for this system to operate under. 


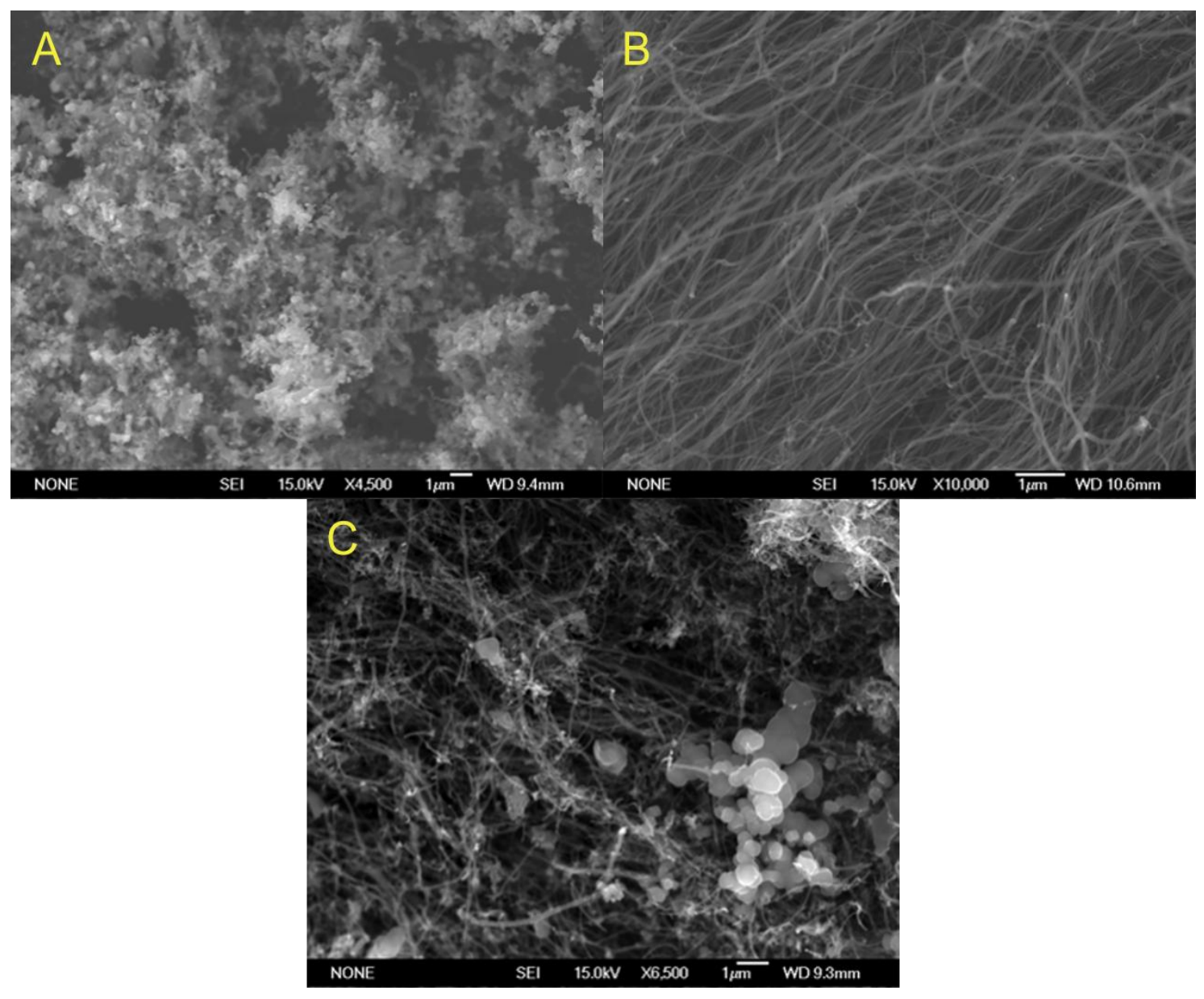

Figure 3-11: Products from CNT deposition at A) $700{ }^{\circ} \mathrm{C}$, B) $800{ }^{\circ} \mathrm{C}$ and C) $900{ }^{\circ} \mathrm{C}$.

\subsection{Effect of gas concentration}

The next parameter investigated was the carbon concentration. Bai and colleagues found that the mole ratio between the catalyst and carbon precursor strongly affected the nature of the carbon products. ${ }^{82}$ They found that when the ratio of ferrocene/benzene was less than $4.2 \%$, carbon nanofibres were favoured; between $4.2 \%$ and $8.8 \%$ MWNTs were favoured; while SWNTs were favoured above $15.1 \%$. To see what effect lowering the carbon concentration would have in this system and to reproduce a $15 \%$ concentration, the temperature of the hexane in the bubbler was lowered to $-6{ }^{\circ} \mathrm{C}$ by immersion in a salt/ice bath. This lowered the vapour pressure 
of hexane to $32 \mathrm{~mm} \mathrm{Hg}$, thus decreasing the concentration of carbon in the precursor vapour. The outcome of this modification is seen in Figure 3-12. The resultant CNT fields were much shorter than previously encountered, measuring 50-100 $\mu \mathrm{m}$. There was also a significant increase in the level of visible impurities. The backscatter image in Figure 3-12B shows these impurities were made of heavier material than carbon, so was likely due to excess iron catalyst. This was confirmed by EDS analysis and is not surprising as the amount of carbon put into this system was lower than in previous experiments, meaning a higher relative amount of iron.

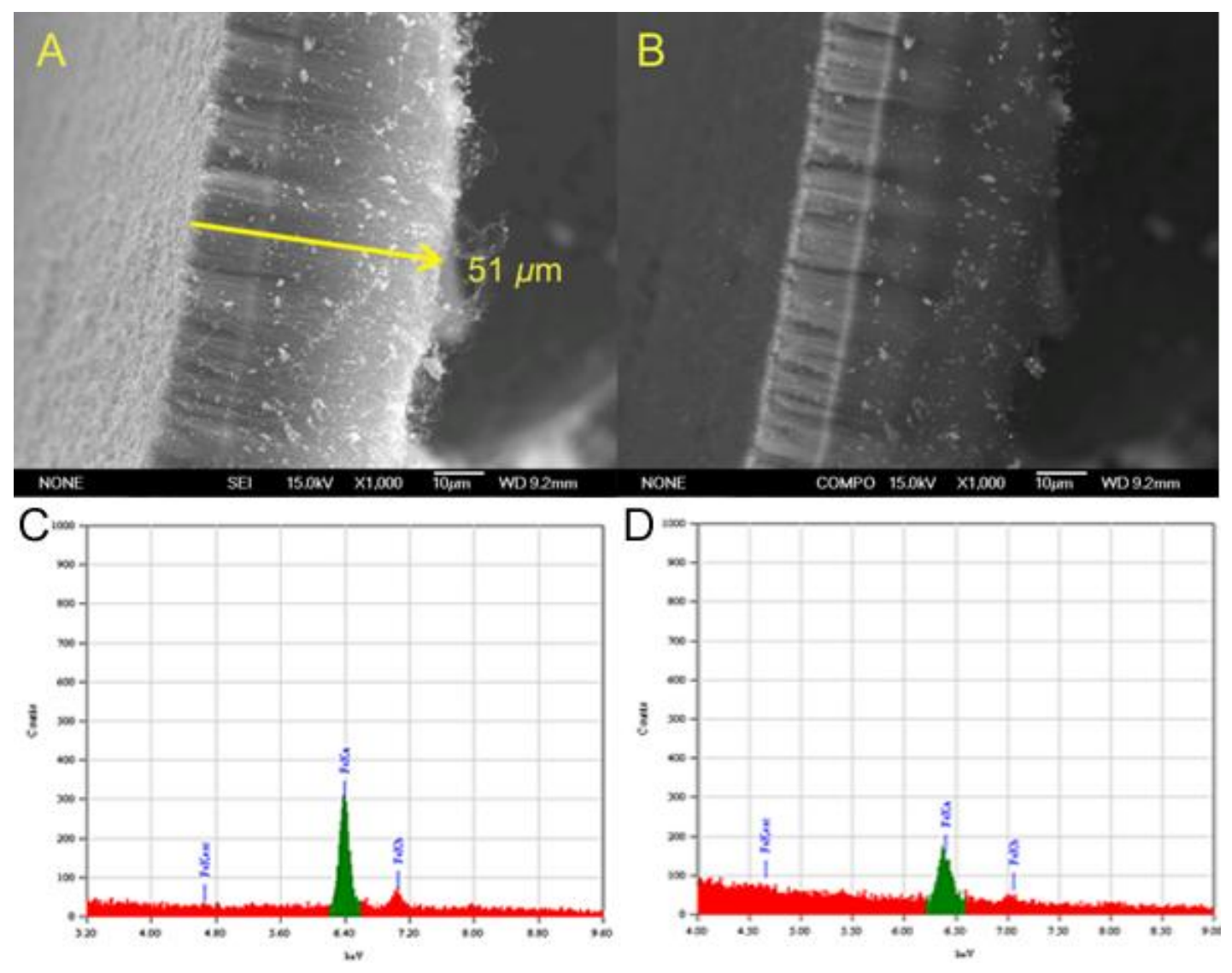

Figure 3-12: A) SEM image of a field of CNTs grown with an iron/carbon ratio of $15 \%$ and $B$ ) the corresponding RBS image with C) an EDS spectrum showing the number of counts of iron in the brighter top region (315 counts) versus D) the EDS spectrum showing the number of counts of iron in the lower darker region (180 counts). 
Interestingly, analysis of the Raman spectrum depicted in Figure 3-13, showed $\frac{\mathrm{I}_{\mathrm{D}}}{\mathrm{I}_{\mathrm{G}}}=$ 0.59 suggesting relatively good graphitisation of the deposits and little evidence of impurities, though it should be noted that Raman analysis is a highly regioselective technique. Also, it revealed no evidence of radial breathing modes, implying there was no SWNT growth. From this test, it was decided that the hexane bubbler should remain at room temperature during synthesis in order to keep the relative level of impurities to a minimum.

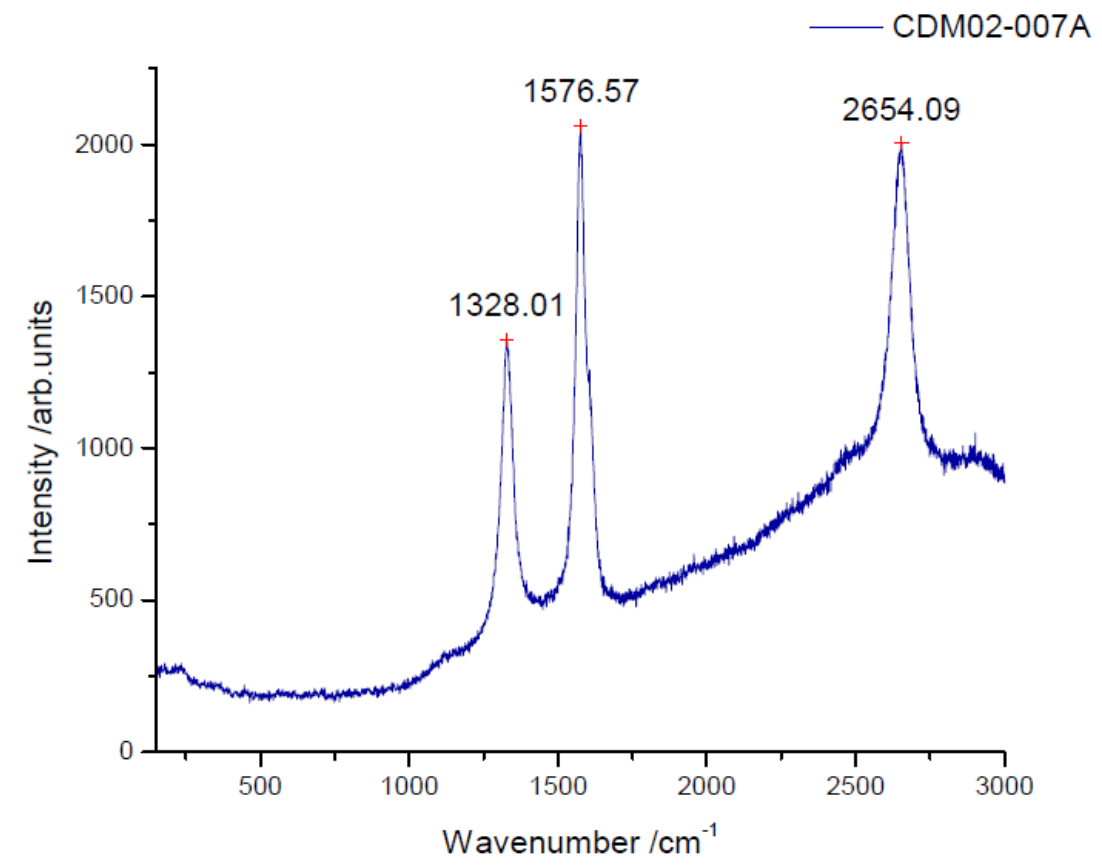

Figure 3-13: Raman spectrum of the products from CNT deposition where the hexane is cooled to $-6{ }^{\circ} \mathrm{C}$.

\subsection{Effect of catalyst}

The next parameter that was investigated was the catalyst. The initial tests employed ferrocene as a volatile organometallic catalyst precursor, which decomposed at high temperature to produce small catalytic iron particles. To further 
examine this factor, other catalysts were used. Firstly, cobalt has shown the ability to preferentially grow SWNTs, so cobaltocene, the cobalt analogue of ferrocene, was investigated for its activity. Figure 3-14 illustrates the products from this modification, where one can see large fields of aligned CNTs were formed. These fields ranged from 50-70 $\mu \mathrm{m}$ in length, much shorter than for the corresponding ferrocene reactions, which tended to range between 100-250 $\mu \mathrm{m}$. This is consistent with other reports that the growth rate of CNTs grown from iron catalysts is twice as high as for cobalt. ${ }^{94}$ From the tube diameters indicated in Figure 3-14B, it is clear that these are also MWNTs. What is interesting is that the distribution of tube ranges appeared smaller than for ferrocene, with no evidence of the very large and very small CNTs seen previously. However, this higher tube uniformity did not lead to the same alignment pattern seen for ferrocene-grown nanotubes. Instead, the fields appeared less well aligned with no discernable alignment pattern. This is likely due to a lower tube density within the fields as a result of the observed slower growth rate, which means that the tubes are not forced into alignment by the close packing of nearest neighbour nanotubes.

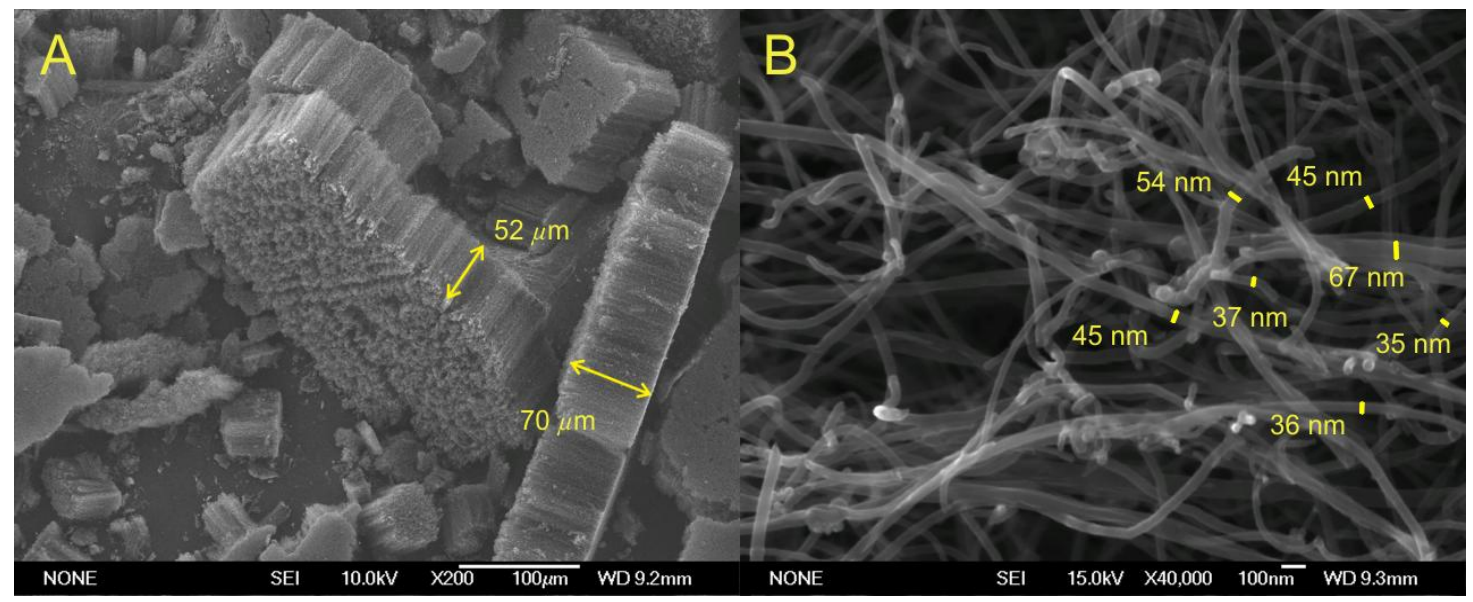

Figure 3-14: SEM images of MWNT products from the decomposition of cobaltocene. 
The next attempt to encourage SWNT growth employed an iron-sulfur catalyst mix. Previous reports have shown that a suitable amount of sulfur in the catalyst will help to promote SWNT growth by providing extra sites for initial CNT nucleation. ${ }^{122,123}$ Zhu and Wei found that an iron/sulfur ratio of 10:1 was suitable for SWNT growth. ${ }^{124}$ Therefore, in this work ferrocene and sulfur were mixed to produce a 10:1 iron/sulfur ratio and employed in CNT growth. The products from this test are illustrated in Figure 3-15. While the products appeared to the naked eye like a metallic film, SEM analysis proved there were some areas of interesting carbonaceous growth. Figure 3-15A shows some disordered CNT growth of different diameters, but what is most striking are the regions with large ribbons of carbon growth. Figure 3-15B depicts an enlarged view of one of these ribbons. One can clearly see that these carbon ribbons have a wave-like pattern, almost corrugated. Evidently the addition of sulfur into the catalyst has affected the way in which carbon precipitates from the catalyst particle, perhaps by altering the way carbon diffuses over the catalyst particle or by altering the particle itself. Ren et al. propose that the sulfur atoms preferentially migrate towards the defect sites in the catalyst particles, thereby repairing these regions and boosting their catalytic activity. ${ }^{122}$ Furthermore, these local iron-sulfur alloy regions within the particle melt at lower temperatures than the surrounding areas. The high catalytic activity means that carbon precipitates preferentially within these local liquid regions, creating nucleation zones. This catalyst particle inhomogeneity could be the cause of the interesting growth seen here. 


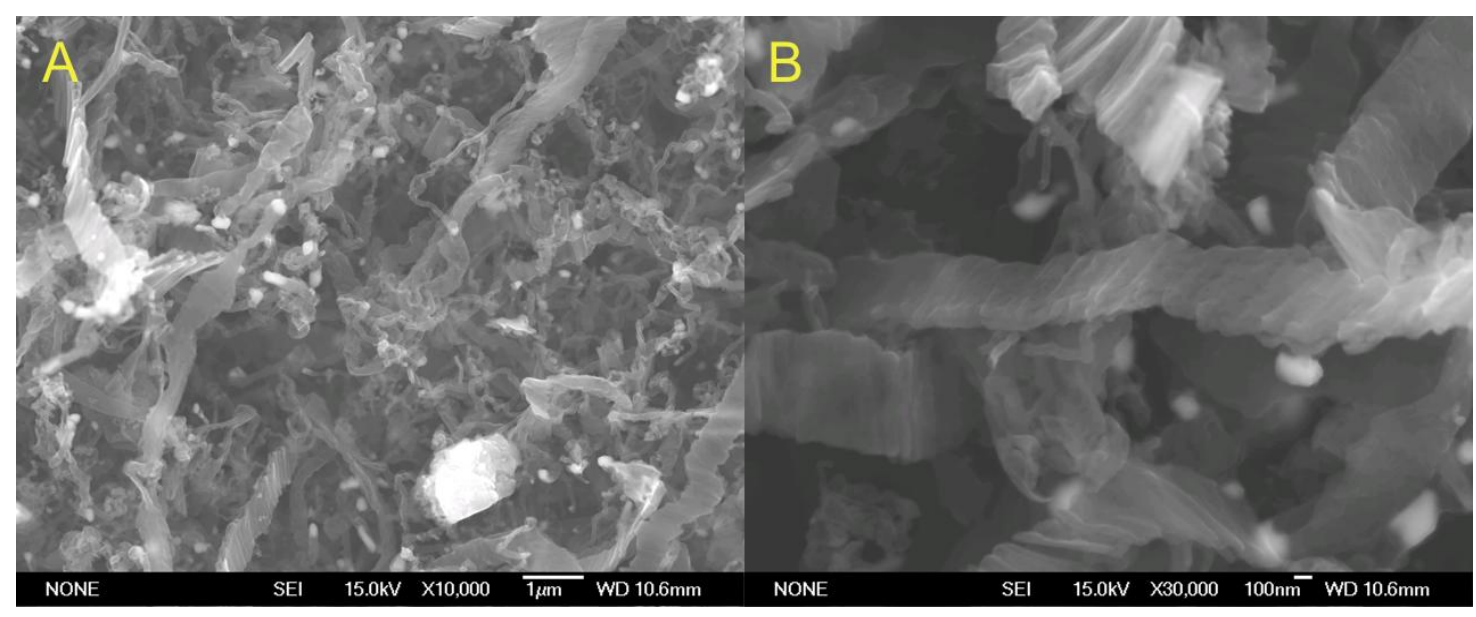

Figure 3-15: SEM images of the products of CNT growth using a 10:1 iron/sulfur catalyst mix.

The next catalyst systems used were metal clusters known as Müller clusters. Müller clusters are large polyoxometalates containing mainly molybdenum as transition metal atom clusters, but other bimetallic clusters containing additional iron and chromium atoms have also been synthesised. Examples of these have been shown previously to produce SWNTs when supported on powdered substrates. Work within this group by Edgar and Spencer showed that iron-molybdenum bimetallic clusters were effective for producing SWNTs, while exclusively molybdenum clusters were not effective for CNT growth. ${ }^{125}$ Additionally, though clusters of different sizes were employed, no relationship between cluster size and tube diameter was apparent.

Here, two different bimetallic Müller clusters were investigated for their activity in CNT growth: these were $\mathrm{Mo}_{72} \mathrm{~V}_{30}$ and $\mathrm{Mo}_{72} \mathrm{Cr}_{30}$. The use of these clusters compelled a change in the catalyst delivery method as they were not sufficiently volatile for gas phase introduction. Instead, samples were drop cast from aqueous solutions onto silicon wafer substrates. Figure 3-17 shows the products after CNT 
reaction using the $\mathrm{Mo}_{72} \mathrm{~V}_{30}$ cluster as a catalyst. The low magnification SEI image in Figure 3-17A depicts products with interesting crystalline morphologies. The EDS maps depicted in Figure 3-17C - E show that these large crystalline products are composed of molybdenum and vanadium, while also appearing to contain carbon. In addition to these large crystallites, smaller molybdenum and vanadium containing particles of various shapes were observed (Figure 3-16). However, there was no evidence of any CNT growth at all. The higher magnification image of one of these crystals illustrated in Figure 3-17B, shows that they are covered in a continuous film of lighter material, determined to be carbon. Furthermore, similar results were observed when $\mathrm{Mo}_{72} \mathrm{Cr}_{30}$ was used as a catalyst, with only continuous films of carbonaceous material deposited. Therefore it was concluded that under these conditions, molybdenum and vanadium and molybdenum and chromium together do not catalyse the growth of CNTs, instead formation of a continuous carbon coating was favoured.

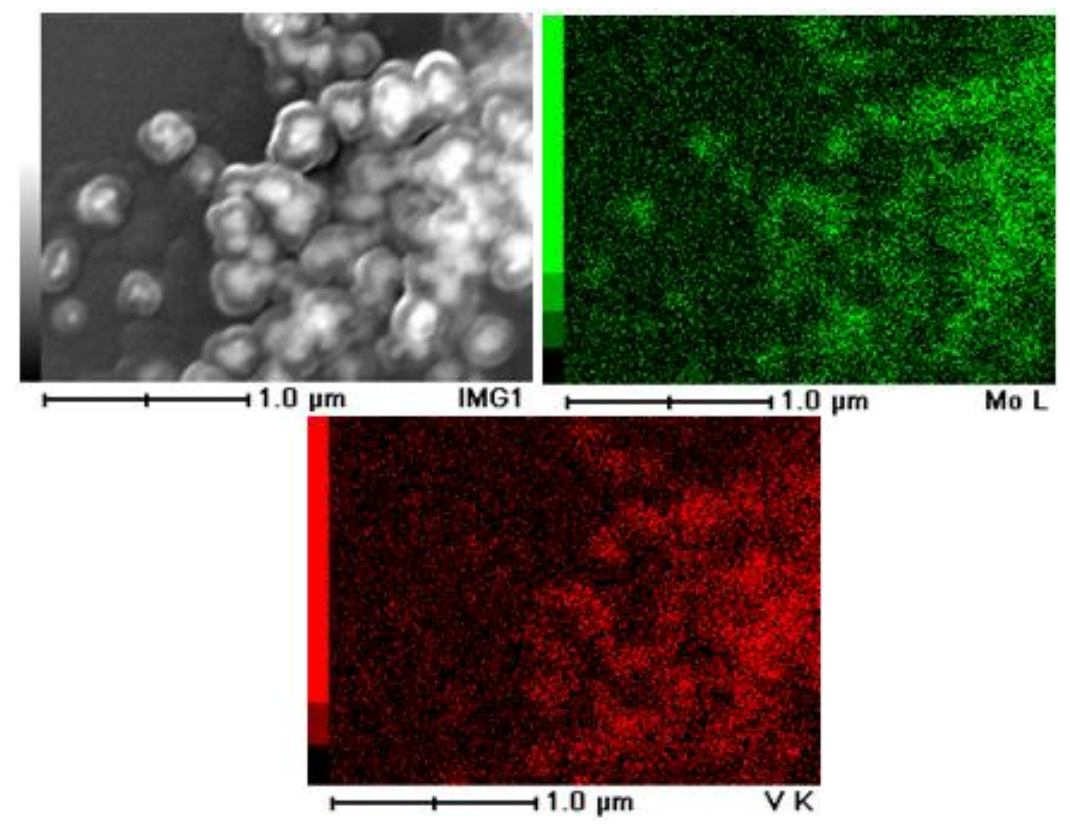

Figure 3-16: EDS images of small molybdenum and vanadium containing particles of various shapes and sizes. 


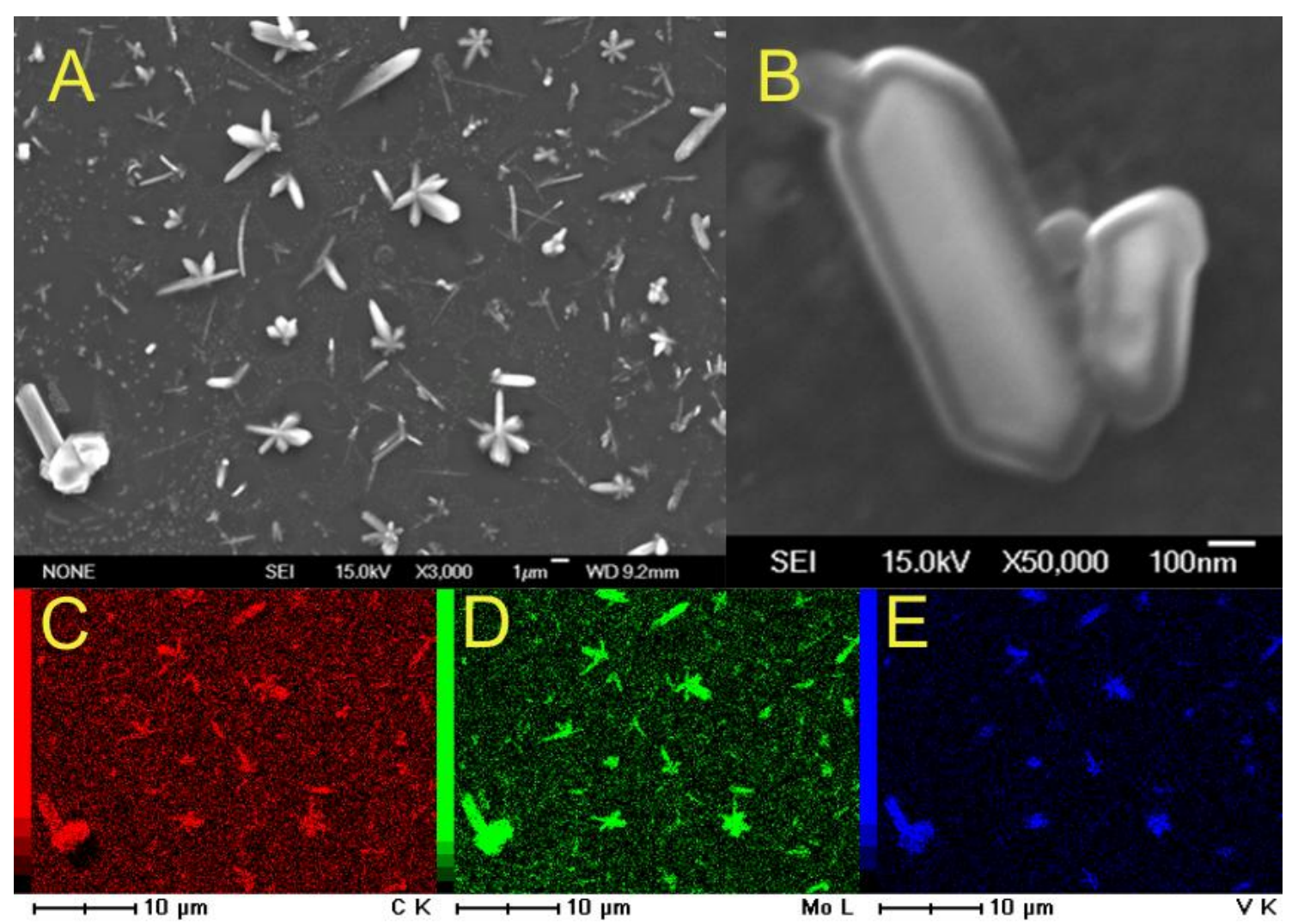

Figure 3-17: SEM images of products from the CNT reaction using $\mathrm{Mo}_{72} \mathrm{~V}_{30}$ as a pre-deposited catalyst. A) Low magnification SEI image of apparently crystalline catalyst deposits with B) a high magnification image of a crystallite. C - E) EDS maps showing the distribution of carbon, molybdenum and vanadium respectively.

\subsection{Effect of water addition}

The final adjustment to the CVD method that was investigated was the addition of water. Hata and colleagues showed that the addition of a small, controlled amount of water vapour greatly enhanced the growth of CNTs, producing large, dense forests of vertically aligned CNTs up to $2 \mathrm{~mm}$ long with $99.9 \%$ carbon purity. ${ }^{126}$ The authors propose that water vapour acts as a very mild oxidant, eliminating the less stable amorphous carbon by oxidation, thereby enhancing the lifetime of the catalyst and leading to the super-long growth of CNTs. 
In this work, water vapour was introduced into the system by adding a water bubbler into the gas inlet system, between the reaction tube and the hexane bubbler. Initial attempts found that the amount of water added at room temperature was far too much, so the temperature of the bubbler was lowered to approximately $15^{\circ} \mathrm{C}$ by immersion in a cold water bath. Figure 3-18 shows the results of this experiment. Again, this process produced large, aligned fields of CNTs, though from RBS images it was clear that there were large amounts of iron catalyst deposited at the top of these fields. This is expected for two reasons: firstly the continuous addition of catalyst throughout the reaction would lead to large deposits of catalyst particles in the uppermost region; and secondly, the elimination of amorphous carbon in this uppermost region by oxidation with water vapour leads to the relatively high amount of iron catalyst. This means that the residual iron catalyst in the top 'crust' is much more visible in this experiment than when compared to results without using water vapour.

The development of a dense top crust of randomly oriented nanotubes has been noted and studied previously by Zhang and colleagues, in the growth of SWNTs by the HiPCo process. ${ }^{127}$ They attribute this top layer to the growth of randomly oriented nanotubes at the start of the growth period. After some time, three minutes in the authors' experiments, the growth switches to a normal base growth mechanism and the top crust is pushed up by vertically aligned nanotube growth. In the authors' study, the top crust exhibits more dense growth in this upper region, evidenced by a brighter region in the SEI image, though it is important to note that they find no evidence of residual catalyst particles. This differs from the present 
study, which finds significant amounts of catalyst in this upper region. This makes it difficult to determine if the large amount of catalyst in the top crust suggests a tip growth model is responsible for the growth of CNTs in these conditions, or if it is just evidence of continued deposition of catalyst particles with limited diffusion.

Thermal analysis, depicted in Figure 3-18D, also showed the absence of amorphous carbon, though the low onset temperature of the major mass loss - at $472{ }^{\circ} \mathrm{C}$ compared with $585{ }^{\circ} \mathrm{C}$ indicated earlier - suggests that the CNTs were not of such high quality as observed previously. This is confirmed by Raman spectroscopy, which showed no evidence of radial breathing modes and revealed $\frac{\mathrm{I}_{\mathrm{D}}}{\mathrm{I}_{\mathrm{G}}}=0.94$, indicating a relatively high level of defects. Hence, it is unlikely that these products contained any SWNTs.

Figure 3-18C shows some interesting doughnut-shaped growth of aligned tubes when looking down at the top face of a large field. These artefacts resemble the cylindrical arrangement observed by Zhao et al. during the water-assisted growth of CNTs. ${ }^{128}$ Though unsure exactly of the mechanism of their growth, the authors attribute this effect to the presence of water. It is interesting to note that these shapes resemble the outline of liquid droplets. Even though at these temperatures water could not exist in condensed form, it is possible that water is selectively adsorbed onto the edges of catalyst droplets, and therefore these regions show enhanced catalytic activity. 


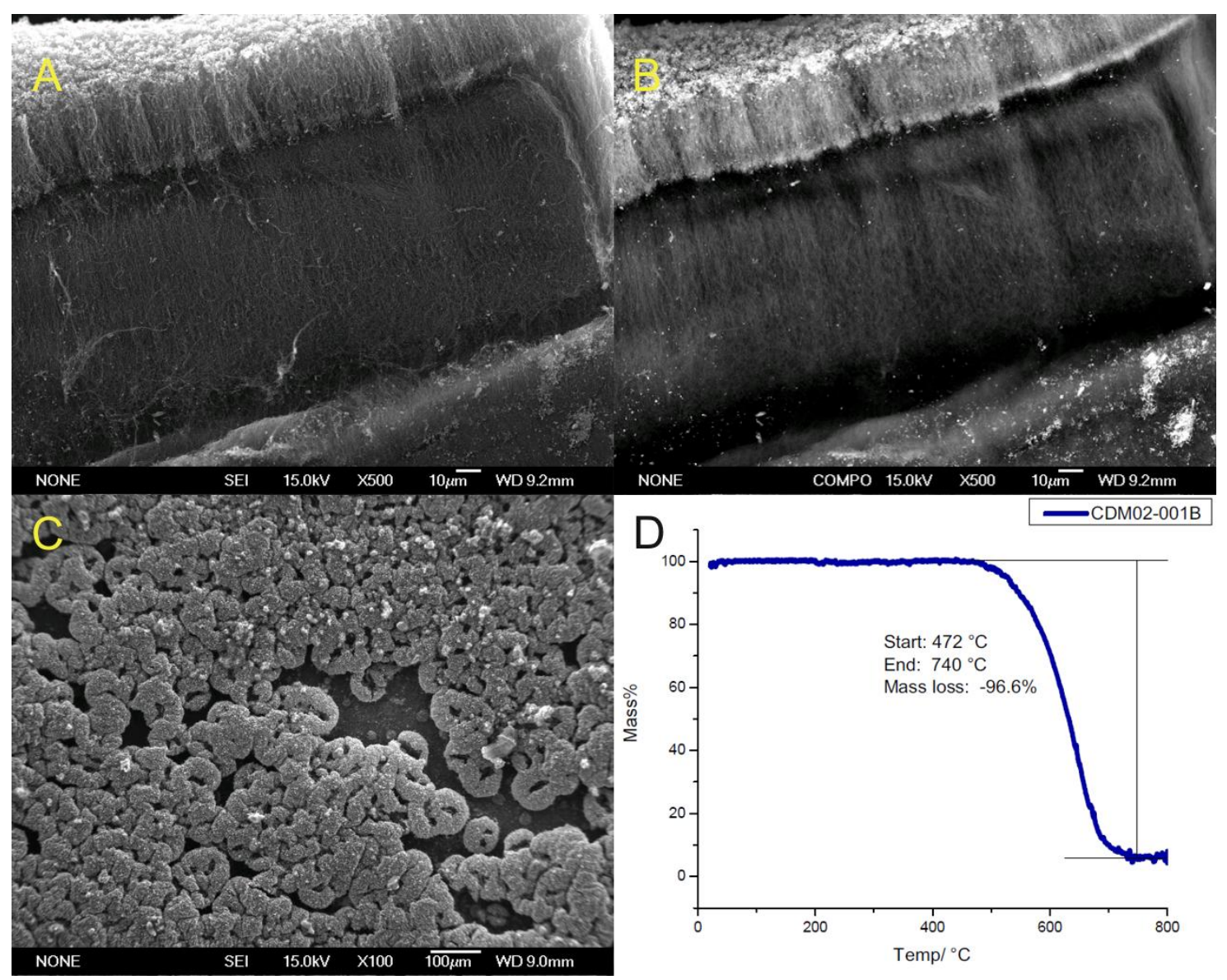

Figure 3-18: A) SEM image of a field of MWNTs and B) the corresponding RBS image illustrating the bright regions at the top of the MWNT field due to iron particles. C) SEM image of the interesting doughnut shaped CNT growth from water and D) the associated thermal analysis.

The poor results seen here are perhaps a result of inadequate control of the amount of water added to the system. Kinetic measurements performed by Yoshihara and co-workers on the water-assisted growth of CNTs suggested that a concentration of water between 1400 and 1700 ppm gives the best results for growth. ${ }^{129}$ In the present work, the concentration of water added was estimated from the vapour pressure of water and varied from approximately $2.2 \%$ or $22,000 \mathrm{ppm}$ at room temperature, to $1.4 \%$ at $15{ }^{\circ} \mathrm{C}$. In order to achieve the desired concentration using this method, the amount of all the other gases would need to be substantially increased, by a factor of 10 . This was not investigated, so it was concluded that 
water addition was unsuitable for this method without significant alteration to the setup.

This chapter has summarised the representative results attained when employing a standard CVD method with hexane and ferrocene as the active precursor species and concludes the efforts to modify the procedure in order to produce SWNTs. In all attempts, MWNTs were the best products obtained. 


\section{Direct Growth}

The potential for using CNT arrays in field emission devices and other electronics makes the study of supported CNTs an important area of research. This chapter looks at the effect of the substrate in the growth of large CNT arrays directly onto monolithic substrates. Having devised a standard floating catalyst CVD method using hexane and ferrocene heated at $800{ }^{\circ} \mathrm{C}$, a number of substrates were examined for their effect on the direct growth of CNTs.

Firstly, the growth on quartz was investigated. Quartz has been used as a CNT support by many groups because of its high thermal stability and the ease with which CNTs can be grown on it. As a consequence of the floating catalyst CVD system employed here, fields of CNTs grow on all faces of the substrate. Figure 4-1 shows the results of one particular CNT growth experiment on a quartz substrate. On the right hand side of Figure 4-1A, one can see a large, vertically aligned field of MWNTs, with a second field of CNTs found on the left. By looking at the shape of the edge of the two fields it is clear that they were once attached, but have likely become separated during SEM sample preparation. These regions, as well as the substrate edges, are often the most useful for imaging as they make it possible to see the field cross section. As observed previously, the CNTs within the cross section of the large fields, depicted in Figure 4-1B, appeared very clean and well aligned, with no visible carbon impurities.

Conversely, looking at the top face of a CNT field in Figure 4-1C, one can see much more irregular CNT growth. The nanotubes here appear to have a much larger spread of diameters and lengths, while growing in random directions. This 
resembles the incomplete growth of CNTs found when very small amounts of catalyst are used, and so is indicative of the early growth of CNTs. As this region is now no longer attached to the substrate, it suggests that in this reaction the growth of CNTs on quartz proceeded through a tip growth mechanism.

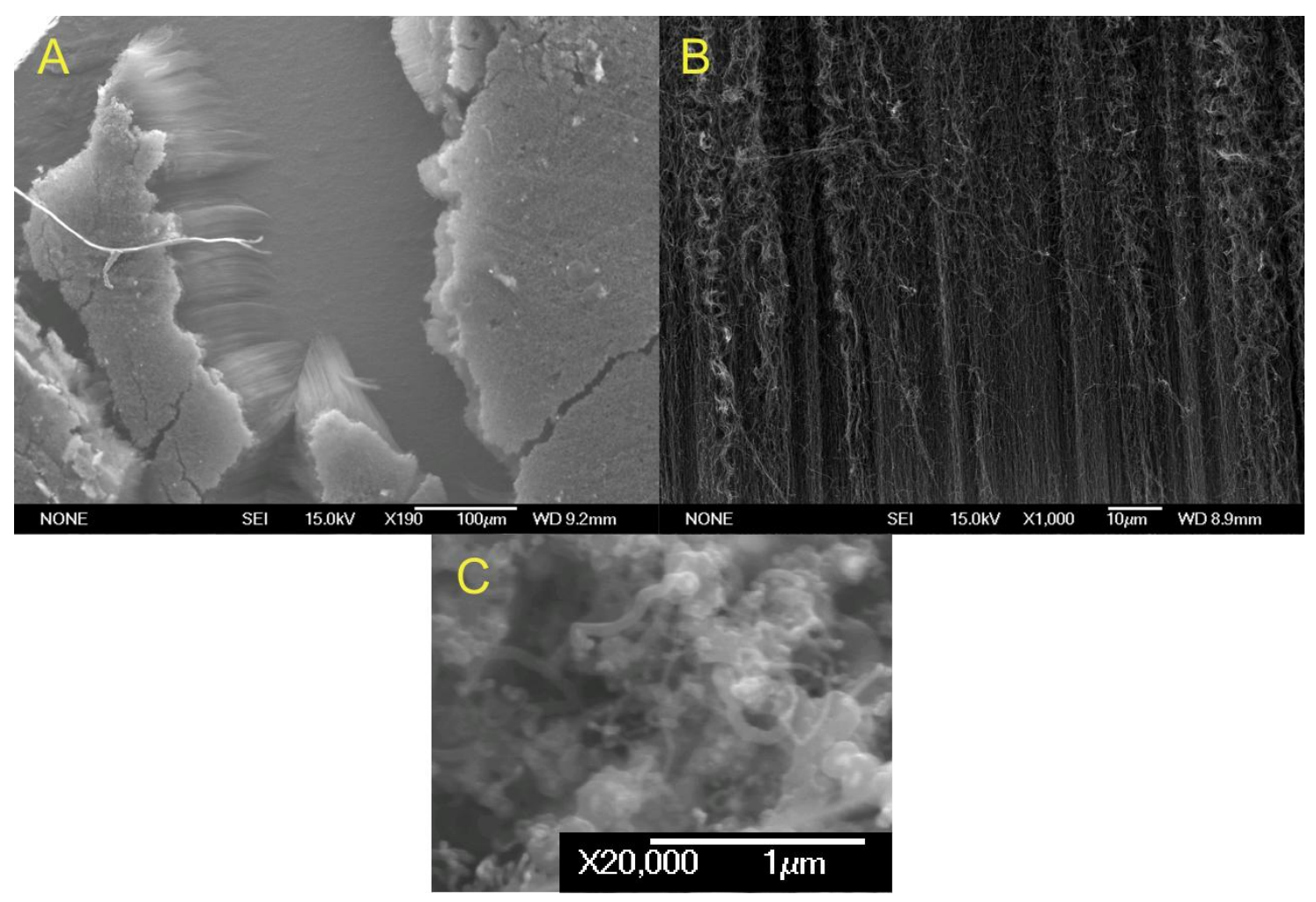

Figure 4-1: A) Low magnification SEM image of the top face of a region of quartz substrate after CNT deposition where the aligned field of CNTs has been scraped away during processing. B) Higher magnification image of a selected cross sectional region of a CNT array and C) of the top surface of a CNT array.

Raman analysis of the products, shown in Figure 4-2, again showed the characteristic D and G bands for MWNT growth at 1328 and $1577 \mathrm{~cm}^{-1}$ respectively, though the $\frac{\mathrm{I}_{\mathrm{D}}}{\mathrm{I}_{\mathrm{G}}}$ was almost one, suggesting a fairly high amount of disorder. The 
presence of iron oxide peaks in the spectrum at $\sim 200 \mathrm{~cm}^{-1}$ is evidence of the high amount of residual iron catalyst situated on the top face of the array.

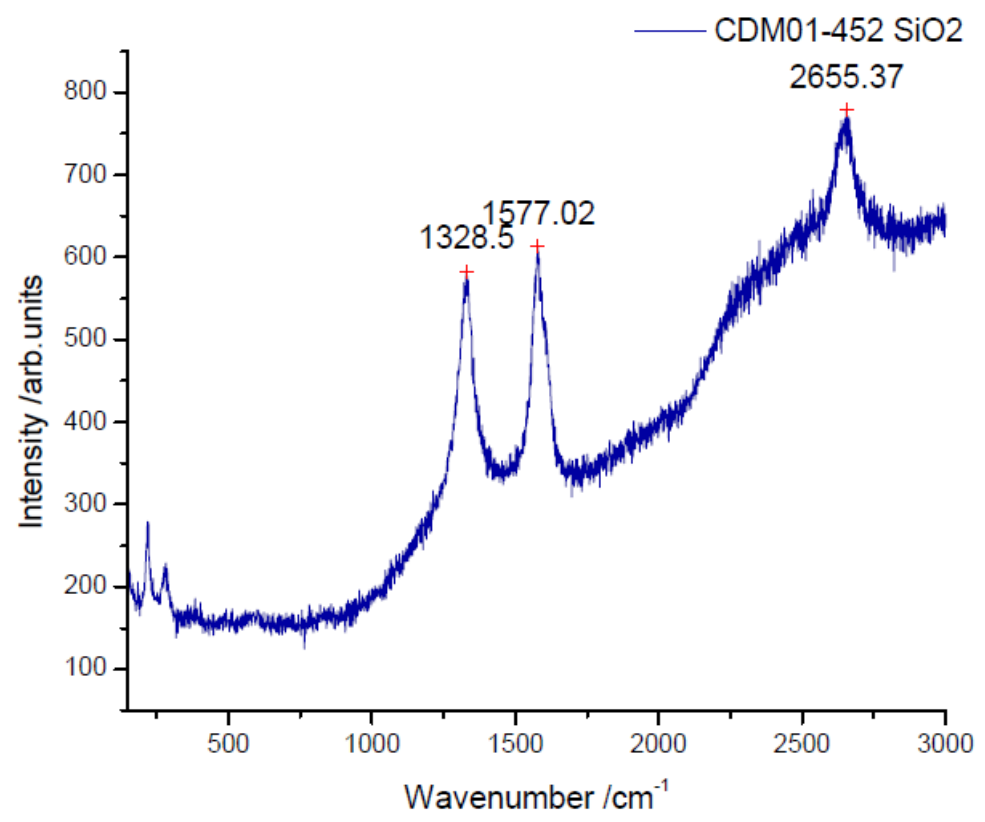

Figure 4-2: Raman spectrum of CNT products deposited on quartz.

Having observed aligned growth on all surfaces of quartz substrates, these were used as a control against which to compare the growth of CNT arrays on other surfaces. A range of further non-conducting, metal oxide substrates were employed including alumina (both crystalline sapphire and amorphous alumina), glazed porcelain, and the aluminosilicate ceramic Pythagoras - and the results compared to those for quartz. Additionally, calcium fluoride was examined as a non-conducting substrate with an alternative surface chemistry. The metal oxides and aluminosilicates all have surface oxides, so could perhaps be expected to behave similarly in their interaction with the catalyst, whereas calcium fluoride has no surface oxides. Analysis of the products from all these substrates demonstrated similar results to quartz for the deposition of carbon, with large, vertically aligned 
fields of MWNTs observed. Representative SEM images of these products are illustrated in Figure 4-3.

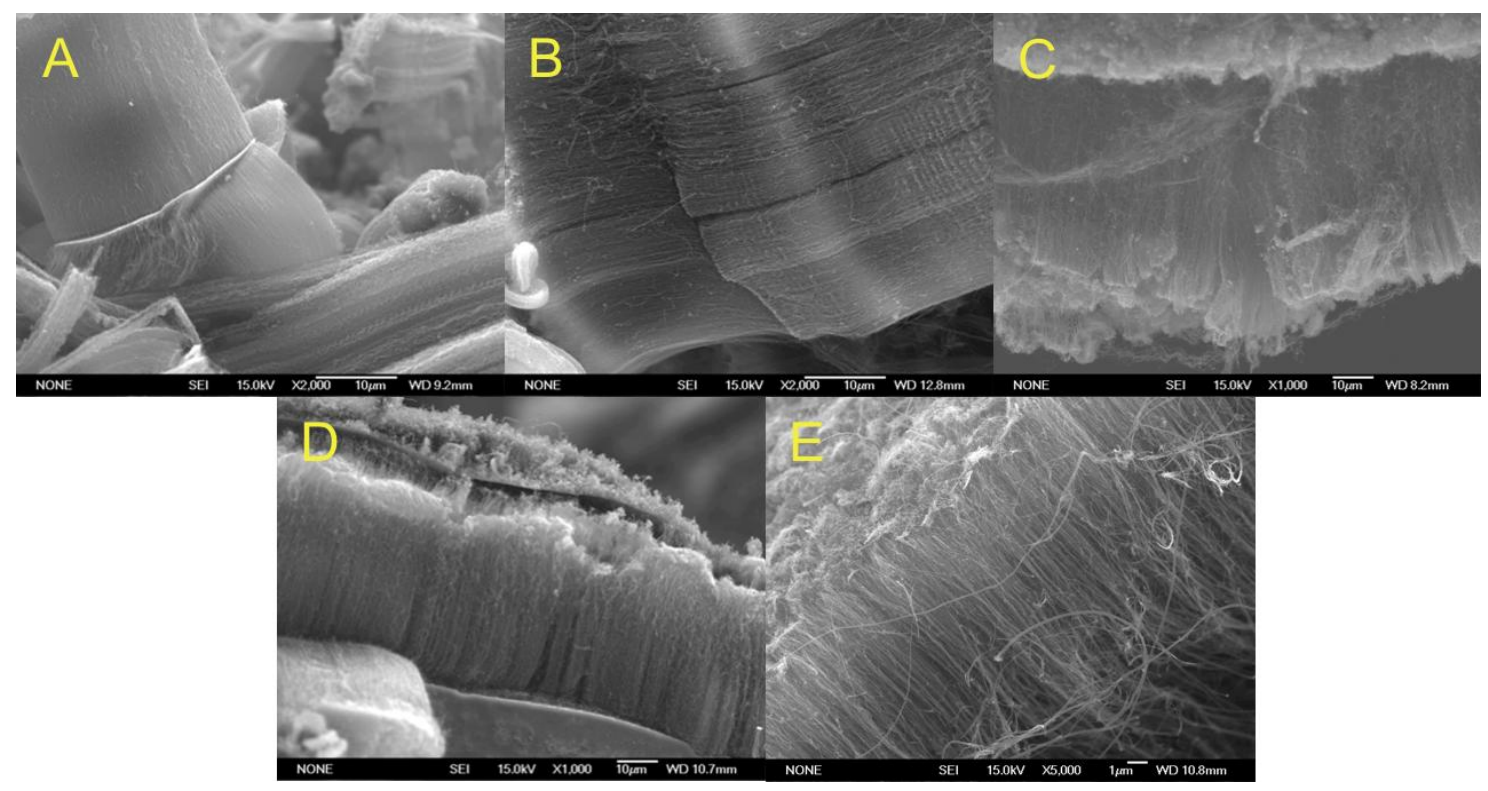

Figure 4-3: SEM images of CNT arrays grown on A) amorphous alumina, B) sapphire, C) Pythagoras, D) glazed porcelain and E) calcium fluoride.

Interestingly, two further non-conducting substrates were examined for their effect on the growth of MWNT arrays. $\beta$ - and $\mathrm{O}^{\prime}$-sialon are silicon-aluminium oxynitrides that are used in high strength applications such as cutting tools. Here, they were investigated for their growth of CNTs as they have a slightly different chemical composition to the metal oxides studied previously. The observed results for CNT deposition for these two substrates was drastically different to those observed previously. From SEM analysis, shown in Figure 4-4, some CNT growth was detected however this was very limited with no large arrays observed. 


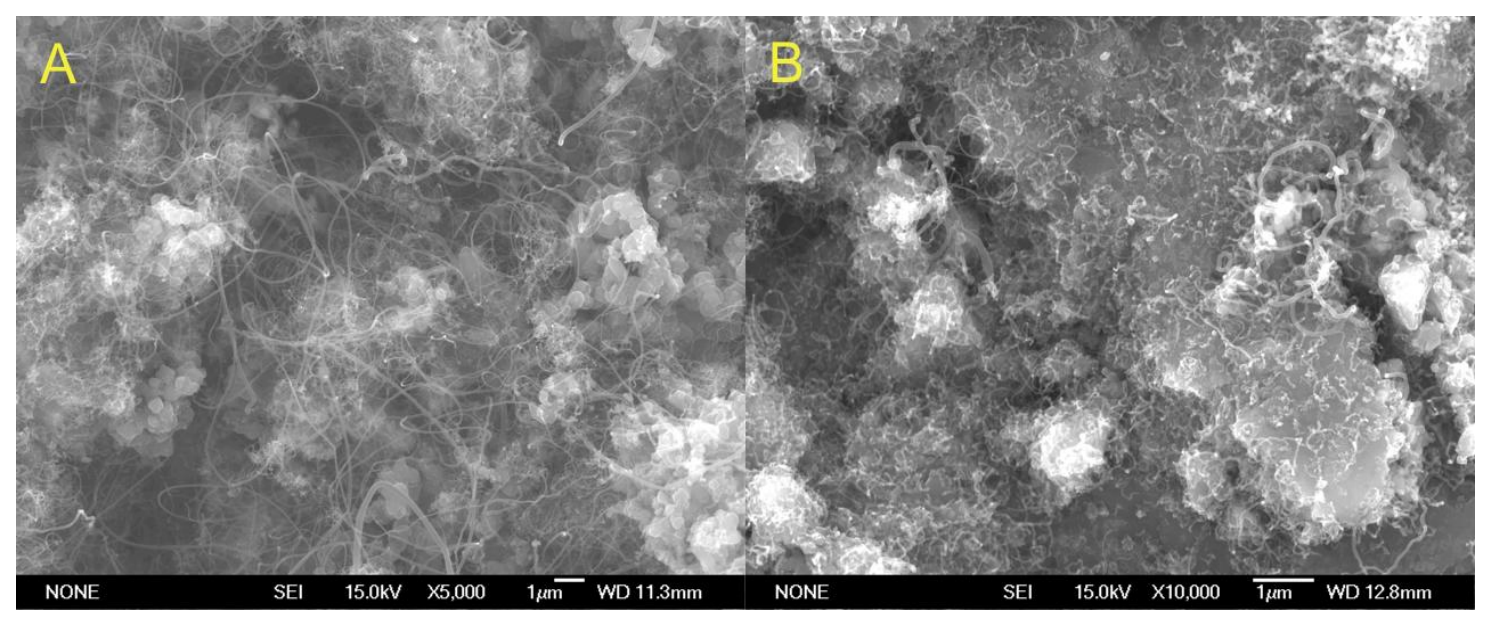

Figure 4-4: SEM images of CNT deposition on A) $\beta$-sialon and B) $O^{\prime}$-sialon.

The first conducting substrate of interest was nickel foil. This was chosen as nickel has been shown to catalyse the deposition of CNTs previously, ${ }^{5,130}$ and as it is a metal it is a good electrical conductor. Few reports of successful growth of CNTs on conducting substrates have been published. ${ }^{130-133}$ In all of these prior reports, the CNT growth is limited and highly irregular. Figure 4-5 depicts the products from the deposition of carbon in the present work. The wide area SEM image in Figure 4-5A shows some interesting growth patterns that were observed on the nickel surface. Firstly, there were ribbons of what appeared to be aligned growth of fields of CNTs. The EDS maps in Figure 4-5D - F show that these regions are highly carbonaceous, obscuring the nickel beneath, but also appear devoid of iron. Closer examination of one of these regions in Figure 4-5B, showed that these regions were composed of amorphous carbon. Given the arrangement of these regions on the nickel surface, it appears that they coincide with the grain boundaries of the underlying substrate. Between these regions, limited growth of CNTs was observed, illustrated in Figure 4-5C. The CNTs were identical to samples obtained previously, being MWNTs of a wide range of diameters. However, these nanotubes were very 
curly, likely a result of the sparse growth, also making it difficult to determine their lengths.

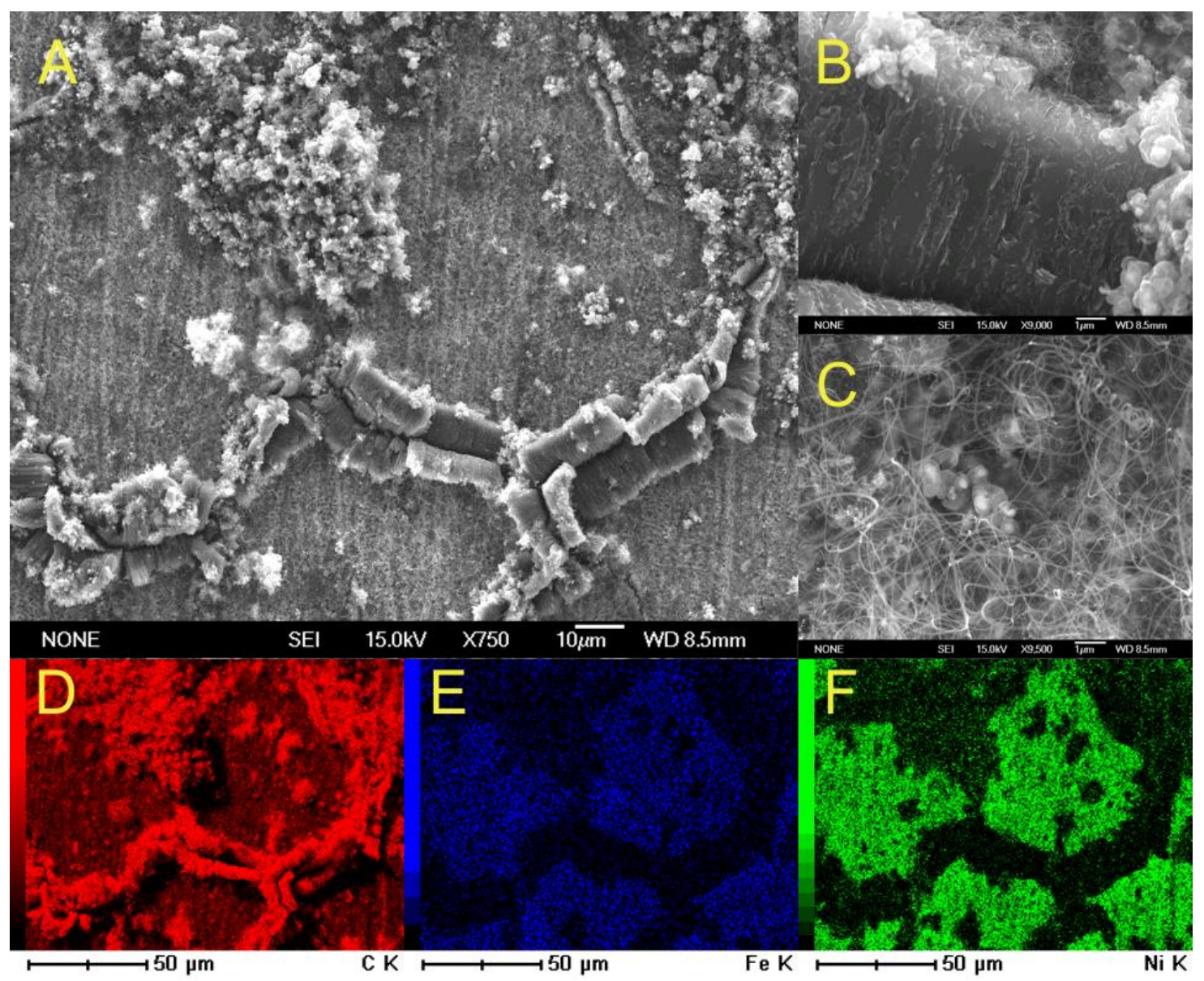

Figure 4-5: Interesting growth patterns on the surface of a Ni substrate.

To explain the observation of these carbonaceous ribbons, it is necessary to consider the distribution of carbon, evident from the EDS maps in Figure 4-5C and D. One can see that the major amount of carbon material is found in the extensive carbonaceous growth, albeit amorphous, at the grain boundaries. A similar phenomenon has been observed previously by Katayama et al., where they found that the growth of MWNTs using a nickel phthalocyanin catalyst on nickel foil, only occurred at micro-defect regions. ${ }^{130}$ This suggested that the growth of CNTs is 
favoured in these defect regions. In the present work there are no CNTs in these similar defect regions, with large amorphous deposits observed instead. However, the morphology of the deposits suggests that these may have been small fields of CNTs that became encased in amorphous carbon as reaction progressed. The regional selectivity is consistent with the preferential deposition of iron catalyst particles in these defect regions, while the increase in amorphous carbon decomposition observed in this work is a consequence of the much higher catalytic activity of iron for the decomposition of hydrocarbon compared with nickel, leading to an excess of active carbon species. ${ }^{94}$

A similar problem was found when CNT deposition was attempted on molybdenum foil. Figure 4-6 shows the results of this study and in the low magnification SEM image, one can see large deposits of amorphous and irregular products. EDS analysis confirmed that these were largely made of carbonaceous material, with smaller amounts of iron. An extensive search of the surface found very few examples of CNT growth; the image in Figure 4-6B is one such region. 


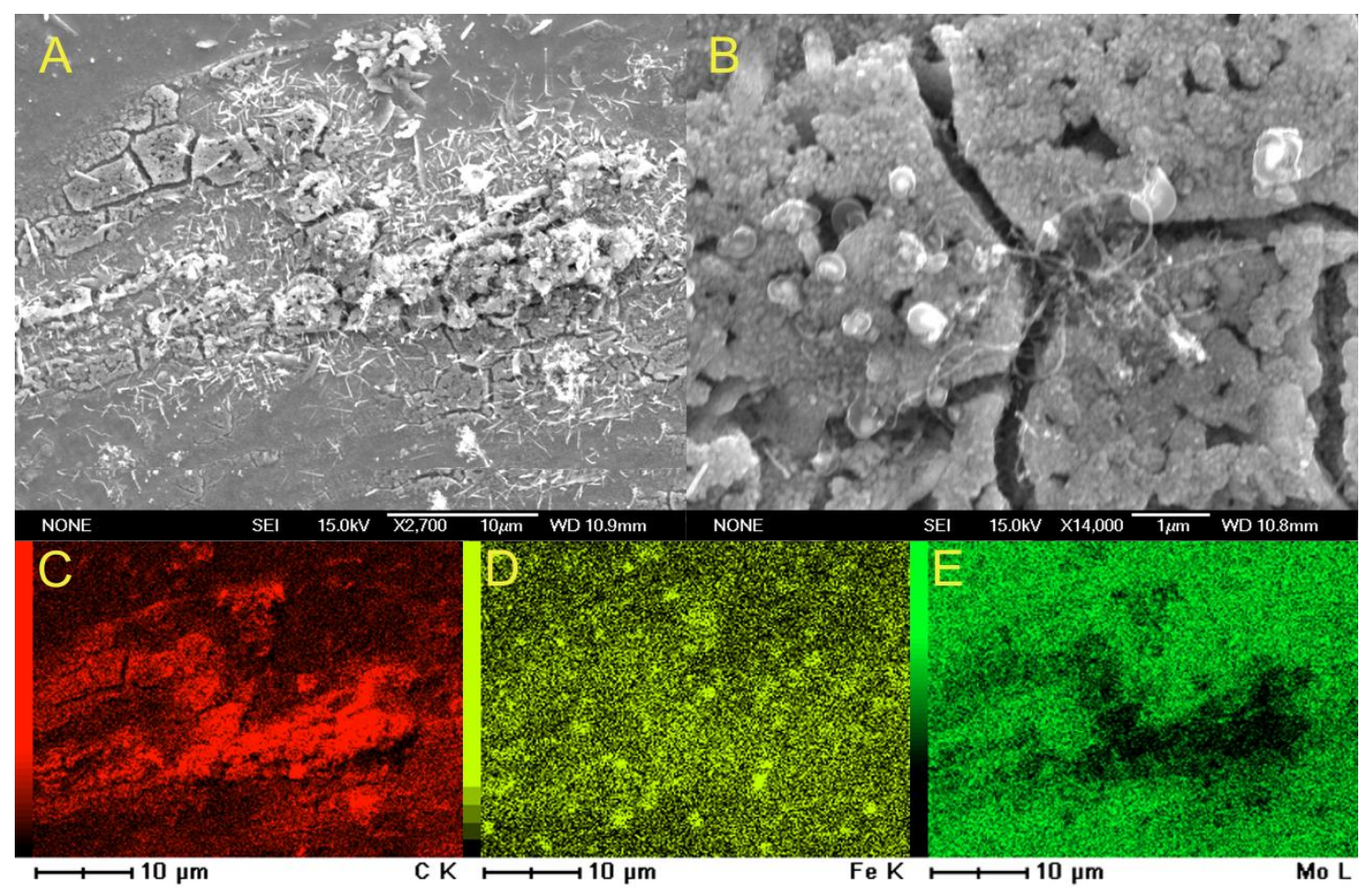

Figure 4-6: SEM images of CNT deposition on the surface of a molybdenum foil substrate.

Following these results, additional conductive substrates were investigated to see if they permitted the growth of large CNT fields. These included highly ordered pyrolitic graphite (HOPG), glassy carbon, and two commercial nickel-iron-siliconboron alloys from Metglas: MBF20 and MBF30, where MBF20 also contains 10$15 \%$ chromium. Highly ordered pyrolitic graphite is a synthetic form of graphite, which is structurally more ordered than natural graphite, with fewer lattice defects and some bonding between the graphene layers. The detailed atomic structure of glassy carbon is less well understood, though it is known that it is not graphitic. ${ }^{134}$ The two alloys were obtained from Metglas, where they are used commercially as brazing foils for filling metal joints at high temperature by capillary action. 
The results of the investigations of these substrates are summarised by the SEM images shown in Figure 4-7. In the case of the two carbon substrates, HOPG and glassy carbon, small amounts of CNTs were observed, however this growth was very limited and sparse. Due to the low density of CNTs, these were able to grow to fill the surrounding space, so that they appeared curly. For the two Metglas substrates, no nanotubes were observed. Instead, the carbon deposits appeared more like a network of irregular particles.

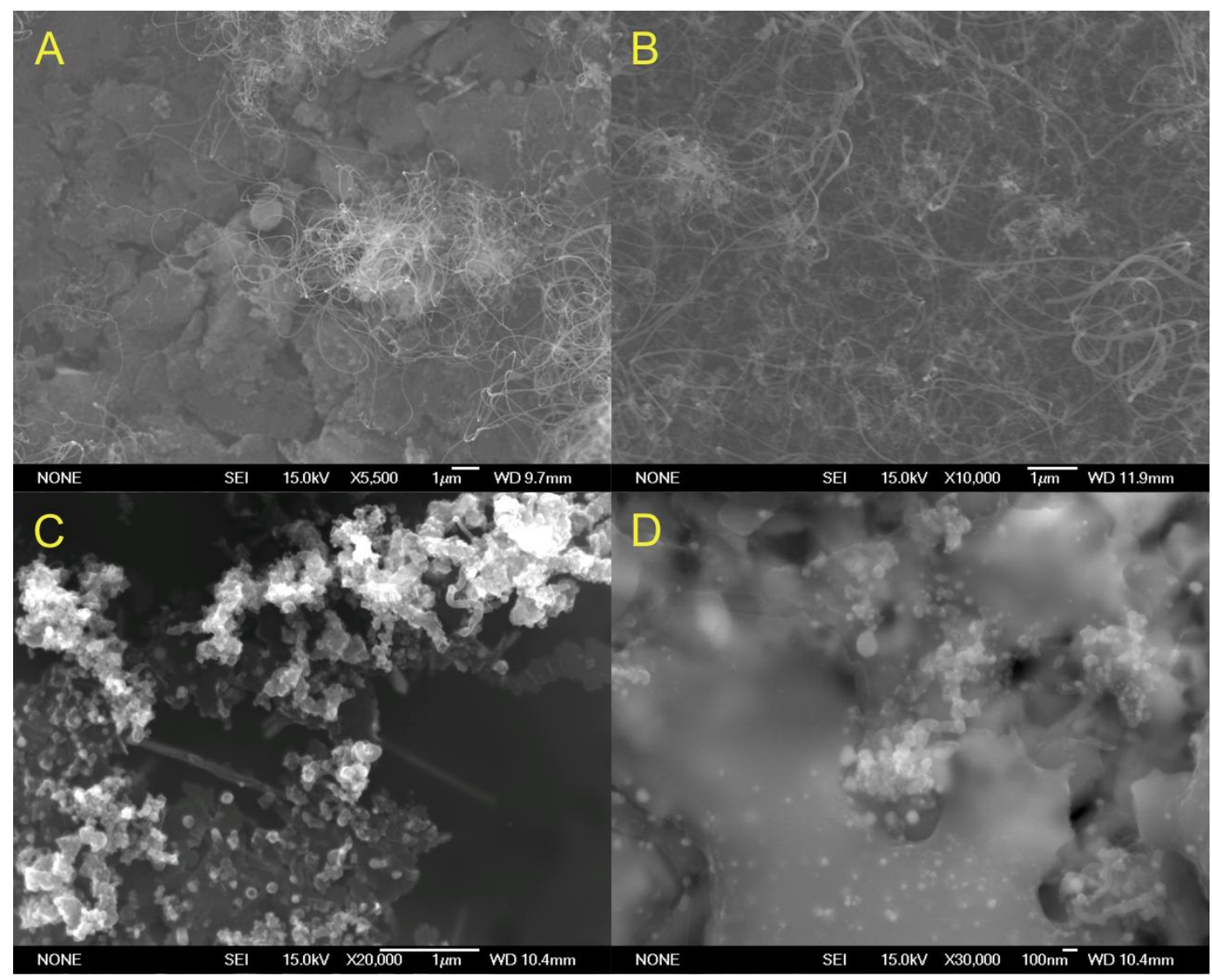

Figure 4-7: SEM images of carbon deposits grown on the surface of A) HOPG, B) glassy carbon, C) MBF20 and D) MBF30. 
To explain the difference between the results for the two groups of substrates - those that do produce CNT arrays (quartz, alumina, porcelain etc.) and those that do not (nickel, molybdenum, HOPG etc.) - it is necessary to consider the substrate-catalyst interaction and the catalyst lifetime. As discussed earlier, for CNT growth to occur an active catalyst particle is required to catalyse both the decomposition of the hydrocarbon precursor and the subsequent growth of a CNT. In the absence of a suitable metal catalyst, at high temperatures the precursor has a tendency to decompose by self-catalysis, leading to the deposition of amorphous carbonaceous materials.

Previous reports have shown that on a substrate the catalyst particles can have a tendency to diffuse into the substrate. Ago et al. found this to be the case for iron particles on a magnesia surface during the growth of SWNTs and DWNTs at temperatures above $500{ }^{\circ} \mathrm{C}$, forming a mixed iron-magnesium-oxide phase. ${ }^{135}$ Chakraborty and colleagues also observed this subsurface diffusion when using iron catalysts to produce CNTs on the surface of silicon wafers at temperatures above $900{ }^{\circ} \mathrm{C} .{ }^{136}$ This subsurface diffusion is a result of strong interactions between the substrate and the metal particles, resulting in low contact angles between the substrate and catalyst. As temperature increases, so too does the rate of diffusion of the particle into the surface so that less remains available to catalyse the growth of CNTs. This leads to a decrease in the rate of CNT growth until eventually there is no catalyst remaining. 


\section{Increasing Temperature}

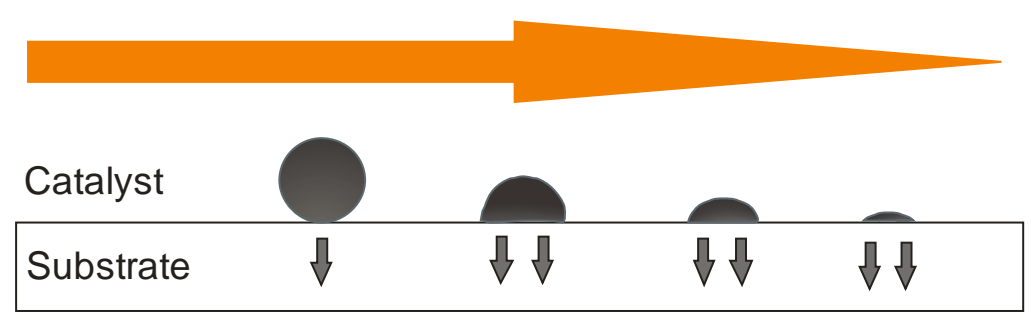

Figure 4-8: Schematic representing the diffusion of catalyst into the substrate leading to catalyst deactivation.

In part, this catalyst deactivation process may help to explain the differences in the growth of large arrays of CNTs on the range of substrates examined. Looking first at the two carbon substrates that were used in the present work, while it was hoped that the presence of an iron catalyst would help the growth of CNTs, it is reasonable to suggest that the substrates themselves could promote the further growth of graphite, in the case of HOPG, and amorphous carbon, in the case of glassy carbon. Therefore, the appearance of a few nanotubes, rather than graphite or amorphous carbon films, is a success in part. The fact that this growth is very limited, as well as the growth for the other substrates that displayed limited CNT growth - nickel, molybdenum, MBF20, MBF30, $\beta$ - and O'-sialon - could be attributed to this phenomenon of subsurface catalyst diffusion, leading to the formation of a series of non-catalytic iron alloys. Aside from the sialon substrates, the other conducting substrates used in the present work have a high affinity for iron, so the subsurface diffusion of the catalyst is highly favoured. Conversely, the metal oxides and aluminosilicates have comparatively weak interactions with iron, so catalyst diffusion is a minor problem and the growth of large fields of CNTs is possible. 
It is a little more difficult to attribute the results of the two sialon substrates to subsurface catalyst diffusion, as the onset temperature of reaction between iron and sialon was found to be $1095{ }^{\circ} \mathrm{C}$ previously, ${ }^{137}$ much higher than the $800{ }^{\circ} \mathrm{C}$ used here. However, it is possible that this system displays a similar size dependent behaviour to the Fe-C system, where it was observed that the temperature of the FeC eutectic between the solid and liquid phases was lowered due to the small catalyst particle sizes. ${ }^{92}$ In the present work, a combination of small iron particle size and high surface roughness of sialon substrates could lower the temperature needed for reaction between the surface and the catalyst.

The rate of catalyst diffusion is highly dependent on the temperature of the reaction system. As this varies along the length of the reaction tube, the rate of catalyst diffusion should be highly dependent on the position of the substrate within the length of the tube. This effect was not examined in the present studies as there were not enough samples of many of the substrate types to test all positions along the tube. However, such as was found with the growth of CNTs on the walls of the silica reaction tube, it is likely that the dependence of the nanotube growth rate (i.e. the rate of precursor decomposition and subsequent nanotube precipitation) on temperature due to its position, is of more significance than the rate of deactivation of the active catalyst. 


\section{Conclusions}

A gas phase thermal CVD technique using hexane and ferrocene as carbon source and catalyst respectively was employed to investigate the growth of CNTs at $800{ }^{\circ} \mathrm{C}$. The first goal of the investigation was to use this floating catalyst technique to fabricate large fields of exclusively SWNTs. Several methods adapted from literature reports were attempted in order to encourage single-walled growth. These included adjusting the operating temperature, employing a mixed iron-sulfur catalyst, using Müller clusters as catalysts, adding water to the process and carefully controlling the iron/carbon ratio. None of these methods was successful, with all attempted modifications only producing MWNTs. This aim clearly needs more careful work to determine the appropriate conditions to produce SWNTs from this technique.

The second aim of the study was to determine the effects of the support material on the growth of the vertically aligned CNT arrays. To this end, several monolithic support materials were tested for the growth of large CNT arrays. It was found that non-conductive, metal oxide materials - including quartz, amorphous alumina, sapphire, glazed porcelain, and the commercial aluminosilicate Pythagoras - as well as fluorite were capable of supporting the growth of extended CNT fields under these conditions.

Conversely, several conducting substrates that were also examined - including nickel, molybdenum, highly ordered pyrolitic graphite and glassy carbon - as well as $\beta$ - and O'-sialon, were unsuccessful in producing large arrays of CNTs. Instead, 
there was some limited CNT growth, which suggests that at some point during the course of the reaction the catalyst loses its ability to make CNTs. This is likely caused by higher iron solubilities with this second group of materials, leading to incorporation of the catalytic species into the support material. This suggests the need for a small buffer layer - silica or alumina, for instance - in order to avoid this catalyst incorporation phenomenon. 


\section{Future Work}

Further work to determine the optimum conditions for SWNT growth using this floating catalyst system is certainly necessary. While the effect of several parameters was investigated, the CVD method has many more variables that can be exploited to control nanotube growth. This could include the gas flow rate, catalyst delivery mechanism and the addition of other additives.

A closer examination of the interaction between the catalyst and the substrate would be useful in understanding the growth of CNT arrays. This could involve the use of X-ray photoelectron spectroscopy (XPS) to determine the bonding of atoms at the surface of the substrate. However, this institution does not have this facility, so to accomplish this access to such an instrument would need to be sought.

Additionally, while the facilities for in situ electron microscopy are not available within this facility, it would advantageous to study the growth of large arrays as a function of time. This could provide insight into the mechanisms of growth, as well as the activity of the catalyst. To do this, the present experimental set-up would need to be modified and a method devised to either isolate or remove substrates conveniently at regular intervals during CNT deposition.

Since the conclusion of experimental efforts in the present work, the metal sputter coater within this institution has been renovated and is now available for use. It would be interesting to examine the growth of many of these conducting substrates when coated with a thin film of a suitable buffer material such as alumina or silica, to see if the production of CNT arrays could be improved. 


\section{References}

(1) Iijima, S., Nature 1991, 354, 56-8.

(2) Radushkevich, L. V.; Luk'yanovich, V. M., Zh. Fiz. Khim. 1952, 26, 88-95.

(3) Baker, R. T. K.; Barber, M. A.; Waite, R. J.; Harris, P. S.; Feates, F. S., J. Catal. 1972, 26, 51-62.

(4) Oberlin, A.; Endo, M.; Koyama, T., J. Cryst. Growth 1976, 32, 335-349.

(5) Boehm, H. P., Carbon 1973, 11, 583-586.

(6) Wiles, P. G.; Abrahamson, J., Carbon 1978, 16, 341-349.

(7) Feynman, R. P. In Annual Meeting of the American Physical Society California Institute of Technology, 1959.

(8) Kroto, H. W.; Heath, J. R.; Obrien, S. C.; Curl, R. F.; Smalley, R. E., Nature 1985, $318,162-163$.

(9) Iijima, S.; Ichihashi, T., Nature 1993, 363, 603-5.

(10) Bethune, D. S.; Kiang, C. H.; de Vries, M. S.; Gorman, G.; Savoy, R.; Vazquez, J.; Beyers, R., Nature 1993, 363, 605-7.

(11) Albertazzi, E.; Domene, C.; Fowler, P. W.; Heine, T.; Seifert, G.; Van Alsenoy, C.; Zerbetto, F., Phys. Chem. Chem. Phys. 1999, 1, 2913-2918.

(12) Hassanien, A.; Tokumoto, M.; Ohshima, S.; Kuriki, Y.; Ikazaki, F.; Uchida, K.; Yumura, M., Appl. Phys. Lett. 1999, 75, 2755-2757.

(13) Dresselhaus, M. S.; Dresselhaus, G.; Eklund, P. C. Science of Fullerenes and Carbon Nanotubes; Academic Press, 1996.

(14) Yakobson, B. I.; Brabec, C. J.; Bernholc, J., Phys. Rev. Lett. 1996, 76, 2511-2514.

(15) Blakslee, O. L., J. Appl. Phys. 1970, 41, 3373-3382.

(16) Lu, J. P., Phys. Rev. Lett. 1997, 79, 1297-1300.

(17) Hernandez, E.; Goze, C.; Bernier, P.; Rubio, A., Phys. Rev. Lett. 1998, 80, 45024505.

(18) Treacy, M. M. J.; Ebbesen, T. W.; Gibson, J. M., Nature 1996, 381, 678-680.

(19) Krishnan, A.; Dujardin, E.; Ebbesen, T. W.; Yianilos, P. N.; Treacy, M. M. J., Phys. Rev. B: Condens. Matter Mater. Phys. 1998, 58, 14013-14019.

(20) Wong, E. W.; Sheehan, P. E.; Lieber, C. M., Science 1997, 277, 1971-1975.

(21) Salvetat, J. P.; Briggs, G. A. D.; Bonard, J. M.; Bacsa, R. R.; Kulik, A. J.; Stockli, T.; Burnham, N. A.; Forro, L., Phys. Rev. Lett. 1999, 82, 944-947. 
(22) Salvetat, J. P.; Kulik, A. J.; Bonard, J. M.; Briggs, G. A. D.; Stockli, T.; Metenier, K.; Bonnamy, S.; Beguin, F.; Burnham, N. A.; Forro, L., Adv. Mater. 1999, 11, 161165 .

(23) Poncharal, P.; Wang, Z. L.; Ugarte, D.; de Heer, W. A., Science 1999, 283, $1513-$ 1516.

(24) Yu, M. F.; Files, B. S.; Arepalli, S.; Ruoff, R. S., Phys. Rev. Lett. 2000, 84, 55525555.

(25) Yu, M. F.; Lourie, O.; Dyer, M. J.; Moloni, K.; Kelly, T. F.; Ruoff, R. S., Science 2000, 287, 637-640.

(26) Kevlar Aramid Fibre - Technical Guide, Du Pont, 2007.

(27) Dresselhaus, M. S.; Dresselhaus, G.; Charlier, J. C.; Hernandez, E., Philos. Trans. R. Soc. London, A 2004, 362, 2065-2098.

(28) Hamada, N.; Sawada, S.; Oshiyama, A., Phys. Rev. Lett. 1992, 68, 1579-1581.

(29) Saito, R.; Fujita, M.; Dresselhaus, G.; Dresselhaus, M. S., Appl. Phys. Lett. 1992, 60, 2204-2206.

(30) Zang, M., IEEE Trans. Nanotechnol. 2005, 4, 452-459.

(31) Blase, X.; Benedict, L. X.; Shirley, E. L.; Louie, S. G., Phys. Rev. Lett. 1994, 72, 1878.

(32) Kane, C. L.; Mele, E. J., Phys. Rev. Lett. 1997, 78, 1932-1935.

(33) Tang, Z. K.; Zhang, L. Y.; Wang, N.; Zhang, X. X.; Wen, G. H.; Li, G. D.; Wang, J. N.; Chan, C. T.; Sheng, P., Science 2001, 292, 2462-2465.

(34) Kong, J.; Franklin, N. R.; Zhou, C. W.; Chapline, M. G.; Peng, S.; Cho, K. J.; Dai, H. J., Science 2000, 287, 622-625.

(35) Collins, P. G.; Bradley, K.; Ishigami, M.; Zettl, A., Science 2000, 287, 1801-1804.

(36) Saito, R.; Dresselhaus, G.; Dresselhaus, M. S., J. Appl. Phys. 1993, 73, 494-500.

(37) Frank, S.; Poncharal, P.; Wang, Z. L.; de Heer, W. A., Science 1998, 280, 17441746.

(38) Poncharal, P.; Berger, C.; Yi, Y.; Wang, Z. L.; de Heer, W. A., J. Phys. Chem. B 2002, 106, 12104-12118.

(39) Chung, D. S.; Park, S. H.; Lee, H. W.; Choi, J. H.; Cha, S. N.; Kim, J. W.; Jang, J. E.; Min, K. W.; Cho, S. H.; Yoon, M. J.; Lee, J. S.; Lee, C. K.; Yoo, J. H.; Kim, J. M.; Jung, J. E.; Jin, Y. W.; Park, Y. J.; You, J. B., Appl. Phys. Lett. 2002, 80, 40454047.

(40) Yaghoobi, P.; Nojeh, A., Mod. Phys. Lett. B 2007, 21, 1807-1830.

(41) Zheng, X.; Chen, G.; Li, Z.; Deng, S.; Xu, N., Phys. Rev. Lett. 2004, 92, 106803.

(42) Kenneth, A. D.; Babu, R. C., Appl. Phys. Lett. 2000, 76, 375-377. 
(43) Xu, Z.; Bai, X. D.; Wang, E. G., Appl. Phys. Lett. 2006, 88, 133107-3.

(44) Chai, G. Y.; Chow, L., Carbon 2007, 45, 281-284.

(45) Govindaraj, A.; Satishkumar, B. C.; Nath, M.; Rao, C. N. R., Chem. Mater. 2000, $12,202-205$

(46) Kiang, C. H.; Choi, J. S.; Tran, T. T.; Bacher, A. D., J. Phys. Chem. B 1999, 103, 7449-7451.

(47) Sloan, J.; Hammer, J.; Zwiefka-Sibley, M.; Green, M. L. H., Chem. Commun. 1998, 347-348.

(48) Flahaut, E.; Sloan, J.; Coleman, K. S.; Green, M. L. H., AIP Conf. Proc. 2001, 2836.

(49) Friedrichs, S.; Meyer, R. R.; Sloan, J.; Kirkland, A. I.; Hutchison, J. L.; Green, M. L. H., Chem. Commun. 2001, 929-930.

(50) Smith, B. W.; Monthioux, M.; Luzzi, D. E., Nature 1998, 396, 323-324.

(51) Hirahara, K.; Bandow, S.; Suenaga, K.; Kato, H.; Okazaki, T.; Shinohara, H.; Iijima, S., Phys. Rev. B 2001, 64.

(52) Smith, B. W.; Luzzi, D. E.; Achiba, Y., Chem. Phys. Lett. 2000, 331, 137-142.

(53) Suenaga, K.; Okazaki, T.; Wang, C. R.; Bandow, S.; Shinohara, H.; Iijima, S., Phys. Rev. Lett. 2003, 90, 055506/1-4.

(54) Kimura, K.; Ikeda, N.; Maruyama, Y.; Okazaki, T.; Shinohara, H.; Bandow, S.; Iijima, S., Chem. Phys. Lett. 2003, 379, 340-344.

(55) Gogotsi, Y.; Libera, J. A.; Guvenc-Yazicioglu, A.; Megaridis, C. M., Appl. Phys. Lett. 2001, 79, 1021-1023.

(56) Gogotsi, Y.; Libera, J. A.; Yoshimura, M., J. Mater. Res. 2000, 15, 2591-2594.

(57) Dillon, A. C.; Jones, K. M.; Bekkedahl, T. A.; Kiang, C. H.; Bethune, D. S.; Heben, M. J., Nature 1997, 386, 377-379.

(58) Davis, J. J.; Green, M. L. H.; Hill, H. A. O.; Leung, Y. C.; Sadler, P. J.; Sloan, J.; Xavier, A. V.; Tsang, S. C., Inorg. Chim. Acta 1998, 272, 261-266.

(59) Ito, T.; Sun, L.; Crooks, R. M., Chem. Commun. 2003, 1482-1483.

(60) Hamwi, A.; Alvergnat, H.; Bonnamy, S.; Béguin, F., Carbon 1997, 35, 723-728.

(61) Pekker, S.; Salvetat, J. P.; Jakab, E.; Bonard, J. M.; Forro, L., J. Phys. Chem. B 2001, 105, 7938-7943.

(62) Delgado, J. L.; de la Cruz, P.; Langa, F.; Urbina, A.; Casado, J.; Navarrete, J. T. L., Chem. Commun. 2004, 1734-1735.

(63) Strano, M. S.; Dyke, C. A.; Usrey, M. L.; Barone, P. W.; Allen, M. J.; Shan, H. W.; Kittrell, C.; Hauge, R. H.; Tour, J. M.; Smalley, R. E., Science 2003, 301, 15191522. 
(64) Peng, H. P.; Reverdy, P.; Khabashesku, V. N.; Margrave, J. L., Chem. Commun. 2003, 362-363.

(65) Tagmatarchis, N.; Georgakilas, V.; Prato, M.; Shinohara, H., Chem. Commun. 2002, 2010-2011.

(66) Chen, S. M.; Shen, W. M.; Wu, G. Z.; Chen, D. Y.; Jiang, M., Chem. Phys. Lett. 2005, 402, 312-317.

(67) Tasis, D.; Tagmatarchis, N.; Bianco, A.; Prato, M., Chem. Rev. 2006, 106, 11051136.

(68) Liu, J.; Rinzler, A. G.; Dai, H. J.; Hafner, J. H.; Bradley, R. K.; Boul, P. J.; Lu, A.; Iverson, T.; Shelimov, K.; Huffman, C. B.; Rodriguez-Macias, F.; Shon, Y. S.; Lee, T. R.; Colbert, D. T.; Smalley, R. E., Science 1998, 280, 1253-1256.

(69) Paradise, M.; Goswami, T., Mater. Design 2007, 28, 1477-1489.

(70) Ebbesen, T. W.; Ajayan, P. M., Nature 1992, 358, 220-222.

(71) Guo, T.; Nikolaev, P.; Rinzler, A. G.; Tomanek, D.; Colbert, D. T.; Smalley, R. E., J. Phys. Chem. 1995, 99, 10694-10697.

(72) Dai, H.; Rinzler, A. G.; Nikolaev, P.; Thess, A.; Colbert, D. T.; Smalley, R. E., Chem. Phys. Lett. 1996, 260, 471-475.

(73) Nikolaev, P.; Bronikowski, M. J.; Bradley, R. K.; Rohmund, F.; Colbert, D. T.; Smith, K. A.; Smalley, R. E., Chem. Phys. Lett. 1999, 313, 91-97.

(74) Selbmann, D.; Bendjemil, B.; Leonhardt, A.; Pichler, T.; Taschner, C.; Ritschel, M., Appl. Phys. A: Mater. Sci. Process. 2008, 90, 637-643.

(75) Cantoro, M.; Hofmann, S.; Pisana, S.; Scardaci, V.; Parvez, A.; Ducati, C.; Ferrari, A. C.; Blackburn, A. M.; Wang, K.-Y.; Robertson, J., Nano Lett. 2006, 6, 11071112.

(76) Jang, Y.-T.; Ahn, J.-H.; Lee, Y.-H.; Ju, B.-K., Chem. Phys. Lett. 2003, 372, 745749.

(77) Juang, Z. Y.; Chien, I. P.; Lai, J. F.; Lai, T. S.; Tsai, C. H., Diamond Relat. Mater. 2004, 13, 1203-1209.

(78) Deck, C. P.; Vecchio, K., Carbon 2005, 43, 2608-2617.

(79) Su, L. F.; Wang, J. N.; Yu, F.; Sheng, Z. M.; Chang, H.; Pak, C., Chem. Phys. Lett. 2006, 420, 421-425.

(80) Chen, Y.; Yu, J., Appl. Phys. Lett. 2005, 87, 033103/1-033103/3.

(81) Hofmann, S.; Sharma, R.; Ducati, C.; Du, G.; Mattevi, C.; Cepek, C.; Cantoro, M.; Pisana, S.; Parvez, A.; Cervantes-Sodi, F.; Ferrari, A. C.; Dunin-Borkowski, R.; Lizzit, S.; Petaccia, L.; Goldoni, A.; Robertson, J., Nano Lett. 2007, 7, 602-608.

(82) Bai, S.; Li, F.; Yang, Q.; Cheng, H.-M.; Bai, J., Chem. Phys. Lett. 2003, 376, 83-89.

(83) Yasuda, A.; Kawase, N.; Mizutani, W., J. Phys. Chem. B 2002, 106, 13294-13298. 
(84) Chiashi, S.; Murakami, Y.; Miyauchi, Y.; Maruyama, S., Chem. Phys. Lett. 2004, 386, 89-94.

(85) Sharma, R.; Iqbal, Z., Appl. Phys. Lett. 2004, 84, 990-992.

(86) Puretzky, A. A.; Geohegan, D. B.; Jesse, S.; Ivanov, I. N.; Eres, G., Appl. Phys. A: Mater. Sci. Process. 2005, 81, 223-240.

(87) Lin, M.; Tan, J. P. Y.; Boothroyd, C.; Loh, K. P.; Tok, E. S.; Foo, Y.-L., Nano Lett. 2006, 6, 449-452.

(88) Kaminska, K.; Lefebvre, J.; Austing, D. G.; Finnie, P., Nanotechnology 2007, 18, $165707 / 1-6$.

(89) Wagner, R. S.; Ellis, W. C., Appl. Phys. Lett. 1964, 4, 89-90.

(90) Saito, Y., Carbon 1995, 33, 979-988.

(91) 2008 .

(92) Harutyunyan, A. R.; Mora, E.; Tokune, T.; Bolton, K.; Rosen, A.; Jiang, A.; Awasthi, N.; Curtarolo, S., Appl. Phys. Lett. 2007, 90, 163120/1-163120/3.

(93) Liu, L.; Fan, S., J. Am. Chem. Soc. 2001, 123, 11502-11503.

(94) Kim, N. S.; Lee, Y. T.; Park, J. H.; Ryu, H.; Lee, H. J.; Choi, S. Y.; Choo, J. B., J. Phys. Chem. B 2002, 106, 9286-9290.

(95) Ding, F.; Rosen, A.; Bolton, K., Chem. Phys. Lett. 2004, 393, 309-313.

(96) Sinnott, S. B.; Andrews, R.; Qian, D.; Rao, A. M.; Mao, Z.; Dickey, E. C.; Derbyshire, F., Chem. Phys. Lett. 1999, 315, 25-30.

(97) Helveg, S.; Lopez-Cartes, C.; Sehested, J.; Hansen, P. L.; Clausen, B. S.; RostrupNielsen, J. R.; Abild-Pedersen, F.; Norskov, J. K., Nature 2004, 427, 426-429.

(98) Hofmann, S.; Kleinsorge, B.; Ducati, C.; Ferrari, A. C.; Robertson, J., Diamond Relat. Mater. 2004, 13, 1171-1176.

(99) Hofmann, S.; Csanyi, G.; Ferrari, A. C.; Payne, M. C.; Robertson, J., Phys. Rev. Lett. 2005, 95, 036101/1-4.

(100) Song, K.; Yu, W. J.; Cho, Y. S.; Choi, G. S.; Kim, D., Nanotechnology 2004, 15, S590-S595.

(101) Thess, A.; Lee, R.; Nikolaev, P.; Dai, H.; Petit, P.; Robert, J.; Xu, C.; Lee, Y. H.; Kim, S. G.; et al., Science 1996, 273, 483-487.

(102) Saito, Y.; Yoshikawa, T.; Bandow, S.; Tomita, M.; Hayashi, T., Phys. Rev. B 1993, 48, 1907-1909.

(103) Hiura, H.; Ebbesen, T. W.; Tanigaki, K.; Takahashi, H., Chem. Phys. Lett. 1993, 202, 509-512.

(104) Jishi, R. A.; Venkataraman, L.; Dresselhaus, M. S.; Dresselhaus, G., Chem. Phys. Lett. 1993, 209, 77-82. 
(105) Kuzmany, H.; Plank, W.; Hulman, M.; Kramberger, C.; Gruneis, A.; Pichler, T.; Peterlik, H.; Kataura, H.; Achiba, Y., Eur. Phys. J. B 2001, 22, 307-320.

(106) Jorio, A.; Saito, R.; Hafner, J. H.; Lieber, C. M.; Hunter, M.; McClure, T.; Dresselhaus, G.; Dresselhaus, M. S., Phys. Rev. Lett. 2001, 86, 1118-1121.

(107) Milnera, M.; Kurti, J.; Hulman, M.; Kuzmany, H., Phys. Rev. Lett. 2000, 84, 13241327.

(108) Kurti, J.; Zolyomi, V.; Kertesz, M.; Sun, G. Y., New J. Phys. 2003, 5.

(109) Dresselhaus, M. S.; Eklund, P. C., Adv. Phys. 2000, 49, 705-814.

(110) Jorio, A.; Saito, R.; Dresselhaus, G.; Dresselhaus, M. S., Philos. Trans. R. Soc. London, A 2004, 362, 2311-2336.

(111) MacLachlan, R. Honours Dissertation, Victoria University of Wellington, 2002.

(112) Abu Mukh-Qasem, R.; Gedanken, A., J. Colloid Interface Sci. 2005, 284, 489-494.

(113) Futaba, D. N.; Hata, K.; Yamada, T.; Mizuno, K.; Yumura, M.; Iijima, S., Phys. Rev. Lett. 2005, 95, 056104/1-056104/4.

(114) Xiang, R.; Luo, G.; Yang, Z.; Zhang, Q.; Qian, W.; Wei, F., Nanotechnology 2007, $18,415703 / 1-415703 / 4$.

(115) Hornyak, G. L.; Grigorian, L.; Dillon, A. C.; Parilla, P. A.; Jones, K. M.; Heben, M. J., J. Phys. Chem. B 2002, 106, 2821-2825.

(116) Puretzky, A. A.; Eres, G.; Rouleau, C. M.; Ivanov, I. N.; Geohegan, D. B., Nanotechnology 2008, 19.

(117) Sampedro-Tejedor, P.; Maroto-Valiente, A.; Nevskaia, D. M.; Munoz, V.; Rodriguez-Ramos, I.; Guerrero-Ruiz, A., Diamond Relat. Mater. 2007, 16, 542-549.

(118) Lee, C. J.; Park, J.; Huh, Y.; Lee, J. Y., Chem. Phys. Lett. 2001, 343, 33-38.

(119) Wood, R. F.; Pannala, S.; Wells, J. C.; Puretzky, A. A.; Geohegan, D. B., Phys. Rev. B: Condens. Matter Mater. Phys. 2007, 75, 235446/1-235446/8.

(120) Maruyama, S.; Kojima, R.; Miyauchi, Y.; Chiashi, S.; Kohno, M., Chem. Phys. Lett. 2002, 360, 229-234.

(121) Kong, J.; Soh, H. T.; Cassell, A. M.; Quate, C. F.; Dai, H. J., Nature 1998, 395, 878881 .

(122) Ren, W. C.; Li, F.; Cheng, H. M., J. Phys. Chem. B 2006, 110, 16941-16946.

(123) Wei, J.; Zhu, H.; Jia, Y.; Shu, Q.; Li, C.; Wang, K.; Wei, B.; Zhu, Y.; Wang, Z.; Luo, J.; Liu, W.; Wu, D., Carbon 2007, 45, 2152-2158.

(124) Zhu, H.; Wei, B., Chem. Commun. (Cambridge, U. K.) 2007, 3042-3044.

(125) Edgar, K.; Spencer, J. L., Curr. App. Phys. 2006, 6, 419-421. 
(126) Hata, K.; Futaba, D. N.; Mizuno, K.; Namai, T.; Yumura, M.; Iijima, S., Science (Washington, DC, U. S.) 2004, 306, 1362-1364.

(127) Zhang, L.; Li, Z.; Tan, Y.; Lolli, G.; Sakulchaicharoen, N.; Requejo, F. G.; Mun, B. S.; Resasco, D. E., Chem. Mater. 2006, 18, 5624-5629.

(128) Zhao, Z.; Qu, J.; Qiu, J.; Wang, X.; Wang, Z., Chem. Commun. (Cambridge, U. K.) 2006, 594-596.

(129) Yoshihara, N.; Ago, H.; Tsuji, M., J. Phys. Chem. C 2007, 111, 11577-11582.

(130) Katayama, T.; Araki, H.; Kajii, H.; Yoshino, K., Synth. Met. 2001, 121, 1235-1236.

(131) Talapatra, S.; Kar, S.; Pal, S. K.; Vajtai, R.; Ci, L.; Victor, P.; Shaijumon, M. M.; Kaur, S.; Nalamasu, O.; Ajayan, P. M., Nat. Nanotechnol. 2006, 1, 112-116.

(132) Parthangal, P. M.; Cavicchi, R. E.; Zachariah, M. R., Nanotechnology 2007, 18, $185605 / 1-185605 / 5$.

(133) Pal, S. K.; Talapatra, S.; Kar, S.; Ci, L.; Vajtai, R.; Borca-Tasciuc, T.; Schadler, L. S.; Ajayan, P. M., Nanotechnology 2008, 19.

(134) Harris, P. J. F., Philos. Mag. 2004, 84, 3159-3167.

(135) Ago, H.; Nakamura, K.; Uehara, N.; Tsuji, M., J. Phys. Chem. B 2004, 108, 1890818915.

(136) Chakraborty, A. K.; Jacobs, J.; Anderson, C.; Roberts, C. J.; Hunt, M. R. C., J. Appl. Phys. 2006, 100, 084321/1-084321/6.

(137) Vleugels, J.; Vandeperre, L.; VanDerBiest, O., J. Mater. Res. 1996, 11, 1265-1276. 